\title{
Uma Abordagem Fuzzy para a Estabilização de uma Classe de Sistemas Não-Lineares com Saltos Markovianos
}

\author{
NATACHe Do Socorro Dias ARrifano
}

Tese apresentada à Escola de Engenharia de São Carlos da Universidade de

São Paulo, como parte dos requisitos para obtenção do título de Doutora em Engenharia Elétrica

Orientadora: Profa. Dra. Vilma Alves de Oliveira 
"Se vocês plantarem uma árvore boa, o fruto dela será bom; mas se vocês plantarem uma árvore má, também o fruto dela será mau, porque é pelo fruto que se conhece a árvore". Jesus Cristo. (Mateus 12,33) 
A DEUS, por todos os dons recebidos.

A minha família, em especial ao meu companheiro Márcio Nemer e a minha mãe Maria Auxiliadôra, pela força e compreensão nos momentos difíceis.

A minha orientadora Vilma Oliveira, pela atenção e paciência e aos colegas, aos atuais e aos que passaram, do Laboratório de Controle - LAC, pela simpatia e pelo compartilhamento de experiências e alegrias.

Aos colegas do Laboratório de Análise Computacional em Sistemas Elétricos - LACO Rodrigo de Andrade Ramos e Ricardo Vasques de Oliveira e também ao Prof. Newton Geraldo Bretas, pela colaboração neste trabalho.

Ao Grupo de Amigas da Cozinha - GRAC, em especial as amigas da República Alternativa: Patrícia, Karla e Kátia, pelos momentos de descontração e alegria.

Ao Departamento de Engenharia Elétrica da Escola de Engenharia de São Carlos EESC, pela contribuição direta ou indireta neste trabalho de pesquisa.

A Fundação de Apoio à Pesquisa do Estado de São Paulo - FAPESP, pela bolsa de estudos e pelo apoio financeiro concedidos. 


\section{Conteúdo}

Lista de Figuras $\quad$ v

Resumo vii

Abstract viii

1 Introdução 1

1.1 Modelagem e Controle de Sistemas Dinâmicos . . . . . . . . . . . . . . 1

1.2 Justificativas e Importância do Trabalho . . . . . . . . . . . . . . . . . 3

1.3 Objetivos do Trabalho . . . . . . . . . . . . . . . . . 6

1.4 Organização do Trabalho . . . . . . . . . . . . . . . . . . 7

2 Modelagem e Controle Fuzzy $\quad 8$

2.1 Noções Básicas de Lógica Fuzzy . . . . . . . . . . . . . . . . . . 8

2.1.1 Conjuntos fuzzy . . . . . . . . . . . . . . . . . . . . . 8

2.1 .2 Operações lógicas . . . . . . . . . . . . . . . . . . . . . . . . . 12

2.1 .3 Inferência fuzzy . . . . . . . . . . . . . . . . . . . . . . . 12

2.1.4 Controladores fuzzy . . . . . . . . . . . . . . . . . . . . 13

2.2 Modelagem e Controle Fuzzy TS . . . . . . . . . . . . . . . . . 15

2.2 .1 Modelagem fuzzy TS . . . . . . . . . . . . . . . . . 17

2.2.2 Construção do modelo fuzzy . . . . . . . . . . . . . . . . . 19

2.2 .3 Controle fuzzy TS . . . . . . . . . . . . . . . . . . . . . 21

2.3 Modelagem e Controle Fuzzy com Saltos Markovianos . . . . . . . . . . 24

2.3.1 Modelagem fuzzy com saltos Markovianos . . . . . . . . . . . . . 25

2.3 .2 Controle fuzzy . . . . . . . . . . . . . . . . . . . . . . 28

3 Estabilidade e Estabilização $\quad 31$

3.1 Estabilidade Estocástica . . . . . . . . . . . . . . . . . . . . . . . . 31

3.2 Estabilização Fuzzy Estocástica . . . . . . . . . . . . . . . . . . 38 
3.2.1 Índices de desempenho no projeto de controle . . . . . . . . . . 40

3.3 Controle Fuzzy de Custo Garantido . . . . . . . . . . . . . . . . . . . . 44

4 Estabilização Fuzzy Robusta $\quad 50$

4.1 Formulação Preliminar do Problema . . . . . . . . . . . . . . . . 50

4.2 Controle Fuzzy de Desempenho $\mathcal{H}_{\infty}$ Robusto . . . . . . . . . . . . . . . 54

5 Representação de um Sistema Elétrico de Potência por um Sistema $\begin{array}{lr}\text { com Saltos Markovianos } & 65\end{array}$

5.1 Considerações Iniciais . . . . . . . . . . . . . . . . . . . . . . 65

5.1 .1 Tipos de SEP's . . . . . . . . . . . . . . . . 66

5.1 .2 Operação de SEP's . . . . . . . . . . . . . . . . . . 67

5.2 Modelagem de um SEP em Esquema de Co-Geração . . . . . . . . . . . 69

5.2 .1 Relações de equilíbrio . . . . . . . . . . . . . . . . . . . 75

5.3 Modelagem e Controle Fuzzy do SEP em Esquema de Co-Geração . . . 75

5.3 .1 Modelagem fuzzy . . . . . . . . . . . . . . . . . . . 76

5.3.2 Projeto de controle fuzzy estabilizante . . . . . . . . . . . . . 81

5.4 Resultados de Simulação . . . . . . . . . . . . . . . . . . . . . 83

6 Conclusão e Proposições para Trabalhos Futuros 98

6.1 Conclusão . . . . . . . . . . . . . . . . . . . . . . . . . . . . . 98

6.2 Propostas para Trabalhos Futuros . . . . . . . . . . . . . . 100

$\begin{array}{ll}\text { A Determinação dos Erros de Aproximação } & 101\end{array}$

A.1 Representação dos Erros de Aproximação em Setores . . . . . . . . . . 101

A.2 Cálculo do erro de aproximação para o sistema de potência MBI em esquema de co-geração . . . . . . . . . . . . . . . . . . . . . 103

Bibliografia 


\section{Lista de Figuras}

Figura 2.1 Função característica do conjunto $\mathbb{S} \ldots \ldots \ldots$

Figura 2.2 Função de pertinência do conjunto fuzzy $\mathbb{A} \ldots \ldots \ldots$

Figura 2.3 Exemplos de funções de pertinência dos conjunto fuzzy das temperaturas de um objeto. . . . . . . . . . . . . . . . 11

Figura 2.4 Resumo das operações da lógica Booleana padrão. . . . . . . . . 12

Figura 2.5 Diagrama de um processo de inferência fuzzy. . . . . . . . . 13

Figura 2.6 Diagrama em blocos de um controlador fuzzy. . . . . . . . . . 14

Figura 2.7 Esquema de projeto de um controlador baseado em sistemas fuzzy

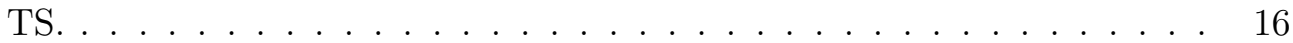

Figura 2.8 Compensação distribuída paralela. . . . . . . . . . . . . . . . . . 22

Figura 2.9 Evolução no tempo de um SNLSM seguindo as mudanças de modo do processo de Markov. . . . . . . . . . . . . . . . . 26

Figura 2.10 Esquema de CDP estendido para o controle do SFSM. . . . . . 29

Figura 5.1 Estrutura básica de um controle do sistema de excitação. . . . . 68

Figura 5.2 Diagrama unifilar do sistema de potência MBI em esquema de co-geração. . . . . . . . . . . . . . . . . . . . . 70

Figura 5.3 Configuração do barramento de cargas do sistema. . . . . . . . . 72

Figura 5.4 Modos de transição do sistema de potência com variações abruptas e aleatórias de cargas. . . . . . . . . . . . . . . . . 73

Figura 5.5 Diagrama em blocos do sistema de controle do SEP. . . . . . . . 76 
Figura 5.6 Funções de pertinência adotadas. . . . . . . . . . . . . . . . . . . . 80

Figura 5.7 Mudanças de condições de operação durante a operação do sistema de potência MBI. . . . . . . . . . . . . . . . . . . . . 83

Figura 5.8 Menu de opções para simulação no Matlab. . . . . . . . . . . . . . 85

Figura 5.9 Diagrama em blocos do PSS. . . . . . . . . . . . . . . . 85

Figura 5.10 Caso 1 - Variáveis de estado: '-' sistema MBI com controle fuzzy, '...' sistema MBI com PSS clássico e '-^-' SFSM com controle fuzzy. . .

Figura 5.11 Caso 1 - Entrada de controle e tensão terminal: '—_' sistema MBI com controle fuzzy, '...' sistema MBI com PSS clássico e ‘-»-' SFSM com controle fuzzy. . . . . . . . . . . . . . . . . . . .

Figura 5.12 Caso 1 - Potências ativa e reativa: '—' sistema MBI com controle fuzzy, '...' sistema MBI com PSS clássico e '- $\diamond-$ ' SFSM com controle fuzzy. 91

Figura 5.13 Caso 2 - Variáveis de estado: '-' sistema MBI com controle fuzzy, ‘...' sistema MBI com PSS clássico e '-§-' SFSM com controle fuzzy. . . 92

Figura 5.14 Caso 2 - Entrada de controle e tensão terminal: '—' sistema MBI com controle fuzzy, ‘...' sistema MBI com PSS clássico e ‘-»-' SFSM com controle $f u z z y \ldots \ldots \ldots \ldots \ldots \ldots \ldots$. . . . . . . . . . . . .

Figura 5.15 Caso 2 - Potências ativa e reativa: '-' sistema MBI com controle fuzzy, '...' sistema MBI com PSS clássico e '- $\diamond-$ ' SFSM com controle fuzzy. 94

Figura 5.16 Caso 3 - Variáveis de estado: '-' sistema MBI com controle fuzzy, ‘...' sistema MBI com PSS clássico e '- $\diamond-$ ' SFSM com controle fuzzy. . .

Figura 5.17 Caso 3 - Entrada de controle e tensão terminal: '- ' sistema MBI com controle fuzzy, ‘...' sistema MBI com PSS clássico e ‘-»-' SFSM com

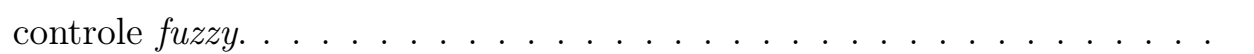

Figura 5.18 Caso 3 - Potências ativa e reativa: '_' sistema MBI com controle fuzzy, '...' 'sistema MBI com PSS clássico e '-ه-' SFSM com controle fuzzy. 97

Figura 5.19 Erro de aproximação: '-, $\left\|\sum_{i=1}^{3} \sum_{j=1}^{2} m_{i}(z) n_{i j}(x) \Delta A_{i j} x\right\|$ e ‘.., $\left\|f(x, r)-\sum_{i=1}^{3} \sum_{j=1}^{2} m_{i}(z) n_{i j}(x) A_{i j} x\right\| \ldots \ldots \ldots$ 


\section{Resumo}

Neste trabalho é apresentada uma abordagem fuzzy para a estabilização de uma classe de sistemas não-lineares com parâmetros descritos por saltos Markovianos. Uma nova modelagem fuzzy de sistemas é formulada para representar esta classe de sistemas na vizinhança de pontos de operação escolhidos. A estrutura deste sistema fuzzy é composta de dois níveis, um para descrição dos saltos Markovianos e outro para descrição das não-linearidades no estado do sistema. Condições suficientes para a estabilização estocástica do sistema fuzzy considerado são derivadas usando uma função de Lyapunov acoplada. O projeto de controle fuzzy é então formulado a partir de um conjunto de desigualdades matriciais lineares. Em adição, um exemplo de aplicação, envolvendo a representação da operação de um sistema elétrico de potência em esquema de co-geração por um sistema com saltos Markovianos, é construído para validação dos resultados.

Palavras-Chave: Controle fuzzy, sistemas fuzzy, estabilidade estocástica, sistemas não-lineares com saltos Markovianos, sistemas com saltos Markovianos, sistemas elétricos de potência, co-geração, estabilizadores de sistemas de potência. 


\section{Abstract}

This work deals with the fuzzy-model-based control design for a class of Markovian jump nonlinear systems. A new fuzzy system modeling is proposed to approximate the dynamics of this class of systems. The structure of the new fuzzy system is composed of two levels, a crisp level which describes the Markovian jumps and a fuzzy level which describes the system nonlinearities. A sufficient condition on the existence of a stochastically stabilizing controller using a Lyapunov function approach is presented. The fuzzymodel-based control design is formulated in terms of a set of linear matrix inequalities. In addition, simulation results for a single-machine infinite-bus power system in cogeneration scheme, whose operation is modeled as an Markovian jump nonlinear system, are presented to illustrate the applicability of the technique.

Keywords: Fuzzy control, fuzzy systems, stochastic stability, Markovian jump nonlinear systems, Markovian jump systems, electrical power systems, co-generation, power system stabilizers. 


\section{Capítulo 1}

\section{Introdução}

\subsection{Modelagem e Controle de Sistemas Dinâmicos}

A modelagem é uma etapa fundamental para o projeto de sistemas de controle tendo em vista que a maioria das técnicas de projeto de controle são baseadas em representações matemáticas dos sistemas reais, também referenciadas como modelos. Na prática, as dinâmicas dos sistemas reais são representadas por um conjunto de equações dinâmicas não-lineares, também conhecido como modelo não-linear, o que dificulta o projeto do controlador. Em geral, os parâmetros do controlador são sintonizados a partir de um modelo linear obtido pela escolha de um ponto de operação e da linearização das equações do sistema neste ponto. Então, o desempenho do controlador é avaliado através de simulações no modelo não-linear para determinadas condições de operação e distúrbios do sistema real. Desta forma, na ocorrência de grandes distúrbios, as condições de operação do sistema podem se desviar significativamente das condições utilizadas no projeto de controle e, conseqüentemente, o sistema deixará de atender às especificações de projeto, podendo até se tornar instável.

Considerando o fato de que o modelo linearizado é obtido para situações idealizadas, existem incertezas relevantes em relação ao modelo não-linear e é desejável que o controlador seja capaz de estabilizar o sistema para as variações esperadas nos seus parâmetros. Além disso, perturbações menos estruturadas no modelo linearizado (por exemplo, em decorrência do truncamento de um modelo complexo, retendo apenas alguns modos dominantes que geralmente operam em uma região de baixa freqüência) também 
devem ser toleradas, sem deteriorar a estabilidade do sistema de controle em malha fechada. Na área de sistemas de controle, estes tipos de incertezas aparecem referenciados como incertezas estruturadas e não estruturadas, respectivamente, e a tolerância a tais incertezas é um problema de estabilidade robusta (Oliveira e Arrifano, 2001; Zhou e Doyle, 1998; Colaneri et al., 1997).

Para atenuar o conservadorismo das soluções de controle disponíveis na literatura, o projeto de controle deve combinar informações quantitativas e qualitativas da operação de um sistema ou processo. As informações qualitativas são geralmente dadas por um especialista na operação e no controle do sistema, enquanto que as informações quantitativas provêm da modelagem do sistema. A combinação de informações qualitativas e quantitativas é a principal motivação para o uso de sistemas inteligentes, ou de inteligência artificial, em sistemas de controle. Os avanços na área de inteligência artificial possibilitaram o surgimento de diversas e inovadoras técnicas que vêm sendo empregadas com sucesso a sistemas reais (não-lineares), tais como, sistemas especialistas (auxiliando a tomada de decisões), redes neurais artificiais (na identificação e monitoração de sistemas), algoritmos genéticos (na otimização de parâmetros de controladores), lógica fuzzy (nas aplicações de controle), etc.

A utilização de lógica fuzzy em sistemas de controle teve início em 1965 com Lofti A. Zadeh (Zadeh, 1965) e se tornou popular nos últimos anos, sendo largamente utilizada principalmente na indústria, ajudando na automação de processos nocivos ao homem. A característica especial da lógica fuzzy é a de representar uma forma inovadora de tratamento de informações imprecisas de forma muito distinta da teoria de probabilidades. A lógica fuzzy provê um método de traduzir expressões verbais, vagas, imprecisas e qualitativas, comuns na comunicação humana, em valores numéricos, possibilitando a conversão da experiência humana em algoritmos que podem ser implementados computacionalmente (Shaw e oes, 1999). Na prática, o uso de lógica fuzzy proporciona o desenvolvimento de ferramentas computacionais flexíveis e eficientes para a solução de problemas complexos, onde é possível a inclusão de conhecimento heurístico.

Os controladores baseados em lógica fuzzy ou simplesmente controladores fuzzy são sistemas baseados em regras que podem ser usados no sistema em malha-aberta ou malha-fechada, quando usados em malha-aberta estabelecem alguns parâmetros de controle de acordo com os quais o sistema deve operar e quando usados em malha- 
fechada baseiam suas ações de controle em medidas das saídas do sistema. Aplicações de controladores fuzzy a processos industriais pertencem a esta última categoria (Wang et al., 1997). Exemplos de aplicações de lógica fuzzy em produtos de consumo e sistemas industriais são encontrados em máquinas de lavar roupas, metrôs, robôs, carros, fábricas de cimento, centrais telefônicas, etc.

Em 1985, Takagi e Sugeno (Takagi e Sugeno, 1985) propuseram uma modelagem fuzzy que deu origem aos controladores fuzzy baseados em modelagem, conhecidos como controladores fuzzy TS. Atualmente, os resultados disponíveis na literatura permitem em vários casos a análise de estabilidade, resposta transitória e robustez de sistemas de controle projetados com base na modelagem fuzzy TS e em ferramentas disponíveis de controle linear que utilizam funções de Lyapunov. Neste contexto, surgiram técnicas de projeto de controle fuzzy mais eficientes do ponto de vista computacional, pois combinam LMI's (do inglês, Linear Matrix Inequalities) (Boyd et al., 1994) e técnicas de programação convexa (Nesterov e Nemirovsky, 1994) na busca de melhores resultados (Tanaka e Wang, 2001).

Observação 1 O termo "fuzzy", literalmente traduzido do inglês como confuso, vago ou impreciso, é usado na área de sistemas de controle como um adjetivo técnico. Da mesma forma que se usa o termo "linear" como adjetivo de sistemas e controle, usa-se o termo fuzzy. Especificamente, os sistemas fuzzy são sistemas precisamente definidos (do ponto de vista matemático) e o controle fuzzy é um tipo especial de controle nãolinear, que também pode ser precisamente definido. Essencialmente, o que se pretende enfatizar é que embora os fenômenos que a teoria de sistemas fuzzy caracteriza possam se imprecisos, a teoria em si é precisa (Wang, 1997).

\subsection{Justificativas e Importância do Trabalho}

A utilização de lógica fuzzy em aplicações de controle tornou-se um campo atrativo de pesquisa nos últimos anos. Particularmente, a representação de equações dinâmicas não-lineares através da modelagem fuzzy TS vem ganhando espaço nas áreas de controle e automação de sistemas e processos, que em geral são controlados em faixas restritas de sua operação. A modelagem fuzzy TS é muito intuitiva e pode ser facilmente compreendida e analisada utilizando conceitos básicos da teoria de controle linear, pop- 
ulares na área de sistemas de controle. Em adição, este tipo de modelagem também apresenta um embasamento matemático, possibilitando o desenvolvimento de critérios sistemáticos de projeto de controle e de análise de estabilidade. Estas características justificam a aceitabilidade da lógica fuzzy tanto na área de pesquisa quanto na área de desenvolvimento de sistemas de controle.

Devido aos avanços nas técnicas computacionais disponíveis, o controle e a análise de estabilidade via LMI's tem sido considerado outro importante campo de pesquisa nos últimos anos. Em relação ao projeto de controladores fuzzy TS, pode-se dizer que este tipo de ferramenta é considerado indispensável e que a busca de soluções menos conservadoras ainda é um forte atrativo para pesquisas neste campo. Vale ressaltar que, mesmo em soluções que reduzem o conservadorismo, é necessário manter o compromisso eficiência $\times$ custo computacional, visto que, as ferramentas mais atraentes usualmente são as mais sistematizadas do ponto de vista computacional, porém a redução do custo computacional nem sempre implica maior eficiência.

Mesmo com o vertiginoso crescimento tecnológico dos últimos anos, ainda existem muitos problemas que precisam ser explorados na área de sistemas de controle, como por exemplo o controle de sistemas sujeitos a variações abruptas e aleatórias. Sabe-se que a classe de sistemas com saltos Markovianos representa muitos sistemas reais que apresentam mudanças abruptas e aleatórias na sua dinâmica. Esta classe de sistemas pode ser modelada utilizando equações dinâmicas lineares ou não-lineares, cujos parâmetros são descritos por saltos Markovianos. Até o momento, os sistemas com saltos Markovianos vêm sendo muito estudados a partir de uma representação linear introduzida por Krasovskii e Lidskii (1961), referenciada como sistemas lineares com saltos Markovianos (SLSM). Como os parâmetros ou até mesmo a estrutura dos SLSM podem ser diferentes em virtude dos saltos, alguns conceitos foram apresentados para o estudo de suas propriedades, tais como os conceitos de estabilidade, estabilização, controlabilidade, observabilidade, dentre outros que podem ser encontrados, por exemplo, em Fang e Loparo (2002), Boukas e Yang (1999a), Feng et al. (1992), Ji e Chizeck (1990), Mariton (1986), Chen (1980), Kamkla e Socha (1977), Sworder e Robinson (1973) e Sworder (1969). Em adição, incertezas na modelagem linear vêm sendo consideradas visando a obtenção de técnicas de estabilização robustas que garantam melhor desempenho. Exemplos destas técnicas podem ser encontrados em Boukas et al. (2003), Costa 
e do Val (2002), Boukas e Liu (2001), Boukas et al. (1999), Boukas e Yang (1999b) e Costa e Boukas (1998). No entanto, para a representação não-linear dos sistemas com saltos Markovianos, os sistemas não-lineares com saltos Markovianos (SNLSM), ao que parece, apenas um trabalho considerando o problema de controle ótimo usando a teoria de programação dinâmica foi registrado (veja Rishel (1975)) e nenhum exemplo de aplicação, até então foi explorado. Isto mostra que o campo de pesquisa para os SNLSM é um campo em aberto, o que permite a proposição não só de novas técnicas de projeto de controle, mas também a construção de exemplos de aplicações envolvendo esta representação de sistemas.

Ainda na área de sistemas de controle, os sistemas elétricos de potência (SEP's) têm atraído a atenção dos pesquisadores devido a grande complexidade de sua operação. Neste campo de pesquisa, um importante tema é o problema das oscilações eletromecânicas. Estas oscilações são prejudiciais à operação dos SEP's, visto que impõem limitações às transferências de potência, o que restringe a utilização da capacidade total de transmissão da rede. Neste contexto, as oscilações podem ser tanto locais, restritas apenas a um gerador, quanto inter-área, envolvendo diversos geradores separados geograficamente. Particularmente quanto ao problema das oscilações locais, os estabilizadores de sistemas de potência (PSS, do inglês Power System Stabilizers) vêm sendo amplamente usados como uma maneira efetiva de enriquecer o amortecimento das oscilações eletromecânicas locais, estendendo os limites de estabilidade dos SEP's (Larsen e Swann, 1981).

Em geral, o efeito das oscilações eletromecânicas é estudado a partir de uma análise modal de uma representação linearizada em torno de apenas um ponto de operação típico do SEP. Por meio desta representação linear, muitas técnicas de projeto de PSS foram desenvolvidas usando, por exemplo, técnicas de análises de sensibilidade e do lugar das raízes (Larsen e Swann, 1981), controle adaptativo (Malik et al., 1986), posicionamento de pólos (Othman e Sanchez-Gasca, 1989) e controle robusto (Werner et al., 2003; Chuanjiang et al., 2003; Rao e Sen, 1999). O principal problema destas técnicas de projeto é o fato de serem baseadas em uma representação linear do SEP, para a qual os parâmetros do PSS são sintonizados. No entanto, atualmente, por razões econômicas e ambientais os SEP's operam cada vez mais próximos de suas capacidades máximas. Desta forma, estes exibem comportamentos fortemente não-lineares, o que, 
conseqüentemente, reduz a eficiência dos PSS's, visto que, na ocorrência de grandes distúrbios as condições de operação mudam de maneira não-linear e os parâmetros do PSS não são mais válidos. Neste caso, o PSS pode até deteriorar a estabilidade do sistema, pela adição de amortecimento negativo ao SEP, por exemplo. Visando superar esta deficiência, algumas técnicas de projeto de controle estabilizante (ou estabilizadores) que consideram as dinâmicas não-lineares dos SEP's têm aparecido, como por exemplo em Guo et al. (2000), Zhu et al. (1997), Wang et al. (1997), Wang e Hill (1996), Wang et al. (1992) e Mark (1992). Além do problema das dinâmicas não-lineares, pode-se considerar os SEP's como sistemas sujeitos a variações abruptas e aleatórias em suas dinâmicas decorrentes de mudanças nos padrões da carga acoplada ao barramento de cargas do sistema. Um sistema operando nesta configuração representa um SEP em esquema de co-geração. Neste contexto, o uso de PSS para redução das oscilações eletromecânicas, ao que parece, ainda não foi investigado, tornando promissor o uso da representação não-linear de sistemas com saltos Markovianos para descrição deste tipo de sistema.

Portanto, tendo em vista o uso bem sucedido da modelagem e controle fuzzy em sistemas não-lineares, a contribuição deste trabalho efetiva-se tanto na campo de pesquisa dos sistemas com saltos Markovianos, quanto no campo dos sistemas de potência através da proposição de uma abordagem de estabilização baseada nas teorias de modelagem e controle fuzzy TS e de estabilização de SLSM, a ser considera em um exemplo de aplicação que representa a operação de um SEP em esquema de co-geração. Sendo portanto este trabalho de pesquisa inovador tanto pela derivação de uma nova abordagem fuzzy de estabilização, como pela sua aplicação em um sistema de potência representado por um SNLSM. Os resultados apresentados neste trabalho aparecem também em Arrifano e Oliveira (n.d.b), Arrifano et al. (2004). Resultados relacionados com a utilização de controle fuzzy aparecem em Arrifano e Oliveira (n.d.a), Oliveira et al. (2003), Oliveira e Arrifano (2003), Arrifano e Oliveira (2002) e Nascimento et al. (2002).

\subsection{Objetivos do Trabalho}

Tendo em vista a construção de um projeto de controle inovador, que seja flexível e robusto do ponto de vista de desempenho, propõe-se neste trabalho uma nova formulação de modelagem e controle fuzzy para a estabilização de uma classe de SNLSM, seguindo a teoria de modelagem e controle fuzzy TS e visando considerar no projeto 
de controle, tanto as não-linearidades, quanto as alterações estocásticas desta classe de sistemas. Esta opção pela modelagem e controle fuzzy TS se justifica pela possibilidade de utilização das propriedades estocásticas de estabilidade e estabilização disponíveis para a classe de sistemas lineares com parâmetros descritos por saltos Markovianos. A técnica de projeto de controle é então formulada em termos de LMI's, viabilizando sua implementação através de ferramentas computacionais atualmente disponíveis. Além da modelagem e projeto de controle fuzzy, propõe-se também a construção de um exemplo de aplicação baseado na operação de um SEP em esquema de co-geração. Mostra-se que, com o uso da abordagem proposta, é possível desenvolver um procedimento de projeto sistemático e eficiente em relação ao desempenho da solução de controle, mesmo considerando o efeito das dinâmicas não-lineares, saltos nos parâmetros e, ainda, a influência de distúrbios externos no sistema.

\subsection{Organização do Trabalho}

O restante do trabalho encontra-se dividido nos seguintes capítulos. No Capítulo 2 são apresentados a modelagem e o controle fuzzy. Nos Capítulos 3 e 4 são apresentadas as condições de estabilidade e estabilização estocástica incluindo o problema de estabilização robusta em termos da modelagem e controle fuzzy propostos. A representação de um SEP em esquema de co-geração por um sistema com saltos Markovianos é tratada no Capítulo 5, onde também são apresentados os resultados de simulação obtidos com o uso da abordagem fuzzy de estabilização. Finalmente, no Capítulo 6 são apresentadas as conclusões deste trabalho e as proposições para trabalhos futuros. Além dos capítulos, ainda é apresentado um apêndice contendo detalhes da obtenção do erro de aproximação usado no projeto de controle fuzzy robusto. 


\section{Capítulo 2}

\section{Modelagem e Controle Fuzzy}

\subsection{Noções Básicas de Lógica Fuzzy}

A lógica fuzzy teve início com base na teoria de conjuntos fuzzy postulada por Zadeh em meados da década de 60. Esta teoria está fundamentada no fato de que muitos fenômenos que ocorrem na natureza não possuem limites ou fronteiras precisamente definidos. Logo, um conjunto fuzzy é um agrupamento impreciso e indefinido, onde a transição de não pertinência para pertinência é gradual e não abrupta como no caso dos conjuntos ordinários definidos pela teoria de conjuntos clássica. Em geral, os conjuntos fuzzy recebem rótulos ou adjetivos que os caracterizam de acordo com a sua faixa de extensão ou variação. Por esse motivo, os conjuntos fuzzy também são denominados de variáveis lingüísticas, ou seja, variáveis cujos valores são palavras em lugar de números. Como exemplo podem ser citados alguns conceitos peculiares à linguagem humana, tais como, quente, morno, muito longe, mais ou menos próximo, altura mediana, meia idade, quase impossível, improvável, etc. A seguir são apresentados alguns conceitos básicos de lógica fuzzy importantes para a compreensão da modelagem e controle fuzzy.

\subsubsection{Conjuntos fuzzy}

Um conjunto fuzzy basicamente é a generalização do conjunto ordinário definido pelo par binário $\{0,1\}$. Entende-se por conjunto ordinário, o conjunto definido a partir da teoria clássica de conjuntos. Um conjunto fuzzy pode ser entendido por um atributo ou grau de pertinência no conjunto, cujo valor exato é parte do intervalo $[0,1]$ em vez 


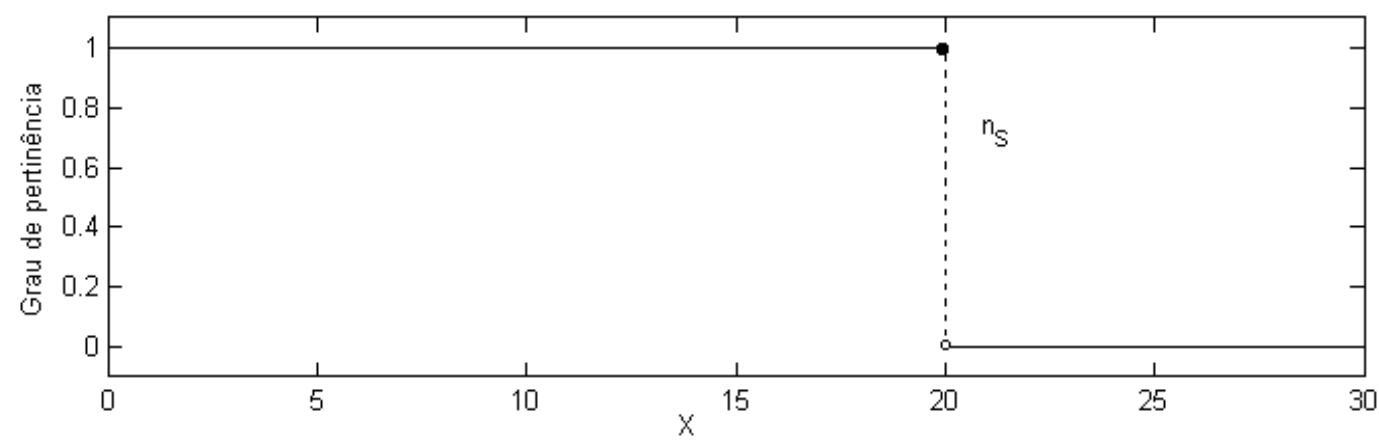

Figura 2.1: Função característica do conjunto $\mathbb{S}$.

de ser parte do conjunto ordinário $\{0,1\}$. Um conjunto ordinário é sempre definido em relação a um universo de discurso, também conhecido como domínio, que também é um conjunto ordinário. Em particular, um conjunto ordinário é definido por uma função característica, que descreve a pertinência dos elementos neste conjunto. Como exemplo, sejam $\mathbb{X}$ o domínio, $\mathbb{S}$ um subconjunto de $\mathbb{X}$, isto é, $\mathbb{S} \subset \mathbb{X}$ e a função característica definida pelo mapeamento $n_{S}: \mathbb{X} \rightarrow\{0,1\}$, tal que, para qualquer elemento $x$ do domínio $n_{S}(x)=1$, se $x$ é um membro de $\mathbb{S}$ e $n_{S}(x)=0$, se $x$ não é um membro de $\mathbb{S}$, ou seja, se $\mathbb{X} \in \mathbb{R}^{+}, \mathbb{R}^{+}$o conjunto dos números reais não-negativos e $\mathbb{S} \subset \mathbb{X}: \mathbb{S}=\{x \in$ $\left.\mathbb{R}^{+} \mid 0 \leq x \leq 20\right\}$, então

$$
n_{S}(x)=\left\{\begin{array}{lll}
1 & \leftrightarrow x \in \mathbb{X} \text { e } x \in \mathbb{S} \\
0 & \leftrightarrow x \in \mathbb{X} \text { e } x \notin \mathbb{S}
\end{array}\right.
$$

A Figura 2.1 ilustra a função característica do conjunto $\mathbb{S}$. Formalmente, os conjuntos fuzzy são definidos como segue.

Definição 1 Um conjunto fuzzy é caracterizado por uma função de pertinência que mapeia os elementos de um domínio, espaço ou universo de discurso $\mathbb{X}$ para o intervalo $[0,1]$, que é (Zadeh, 1965)

$$
A: \mathbb{X} \rightarrow[0,1]
$$

Desta forma, seja $n_{A}$ a função característica do conjunto fuzzy $\mathbb{A}$, então $n_{A}$ caracteriza o mapeamento dos elemento do conjunto $A$ no domínio $\mathbb{X}$ que é dada por $n_{A}: \mathbb{X} \rightarrow[0,1]$. Na teoria de conjuntos fuzzy, a função característica recebe o nome de função de pertinência. Esta terminologia força a idéia de que para cada elemento 


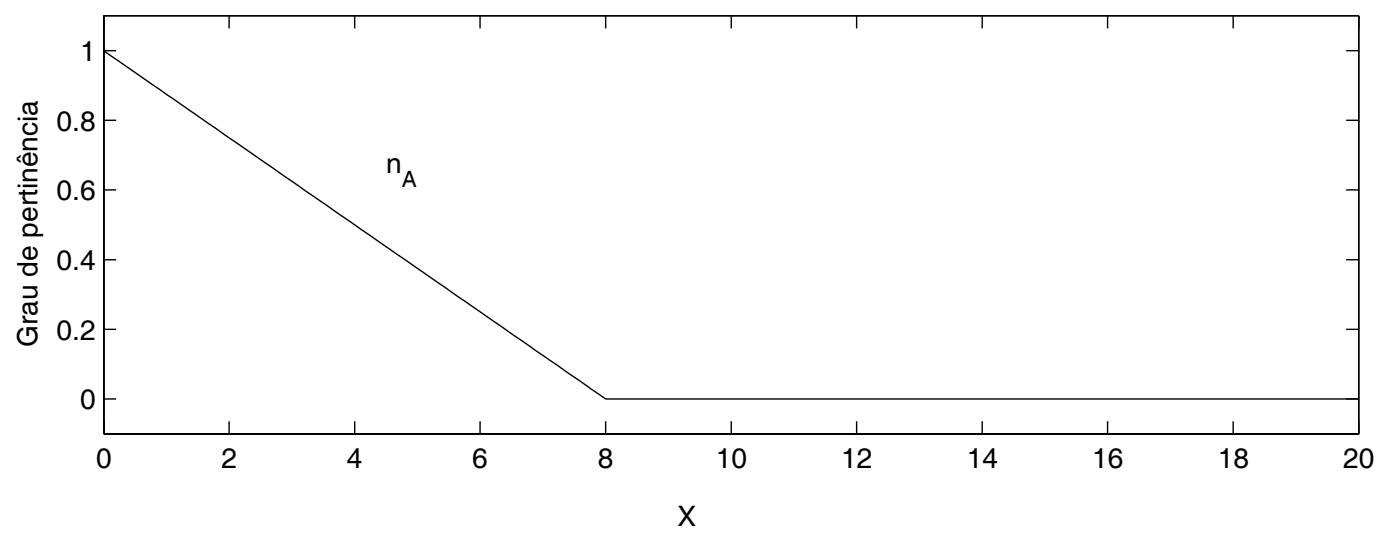

Figura 2.2: Função de pertinência do conjunto fuzzy $\mathbb{A}$.

$x, n_{A}(x)$ indica o grau de que $x$ é um membro do conjunto $\mathbb{A}$. Exemplos típicos de conjuntos fuzzy cujas de funções de pertinência são definidas no domínio dos números reais $\mathbb{R}$ são

$$
n_{A}(x)=\frac{1}{1+x^{2}}
$$

$\mathrm{Ou}$

$$
n_{A}(x)=e^{-(x-5)^{2}}
$$

A Figura 2.2 ilustra outro tipo de função de pertinência de um conjunto fuzzy definido no domínio dos números reais $\mathbb{R}$. Em situações onde o universo do discurso não seja o conjunto dos números reais $\mathbb{R}$ a função de pertinência deve ser expressa em termos dos elementos deste universo. No caso do universo de discurso finito e discreto $\mathbb{X}=$ $\left\{x_{1}, x_{2}, x_{3}, \ldots, x_{n}\right\}$, o conjunto fuzzy é dado na forma de um somatório de elementos que denotam os graus de pertinência dos correspondentes elementos de $\mathbb{X}$, ou seja, $A=\left\{\left(n_{A}\left(x_{i}\right) \mid x_{i}\right) \mid x_{i} \in \mathbb{X}\right\}$, descrito por (Zadeh, 1965)

$$
A=n_{A}\left(x_{1}\right)\left|x_{1}+n_{A}\left(x_{2}\right)\right| x_{2}+\cdots+n_{A}\left(x_{n}\right)\left|x_{n}=\sum_{i=1}^{n} n_{A}\left(x_{i}\right)\right| x_{i} .
$$

Em (2.4) o somatório não deve ser confundido com uma adição algébrica, este somatório denota a união fuzzy de pares ordenados. Como exemplo, seja o universo de discurso $\mathbb{X}=\{1,2,3,4,5\}$ e o conjunto fuzzy $A=0.1|1+0.4| 3+0.6|4+1.0| 5$, então os graus de pertinência dos elementos $1,2,3,4$ e 5 são $0.1,0,0.4,0.6$ e 1.0, respectivamente. 


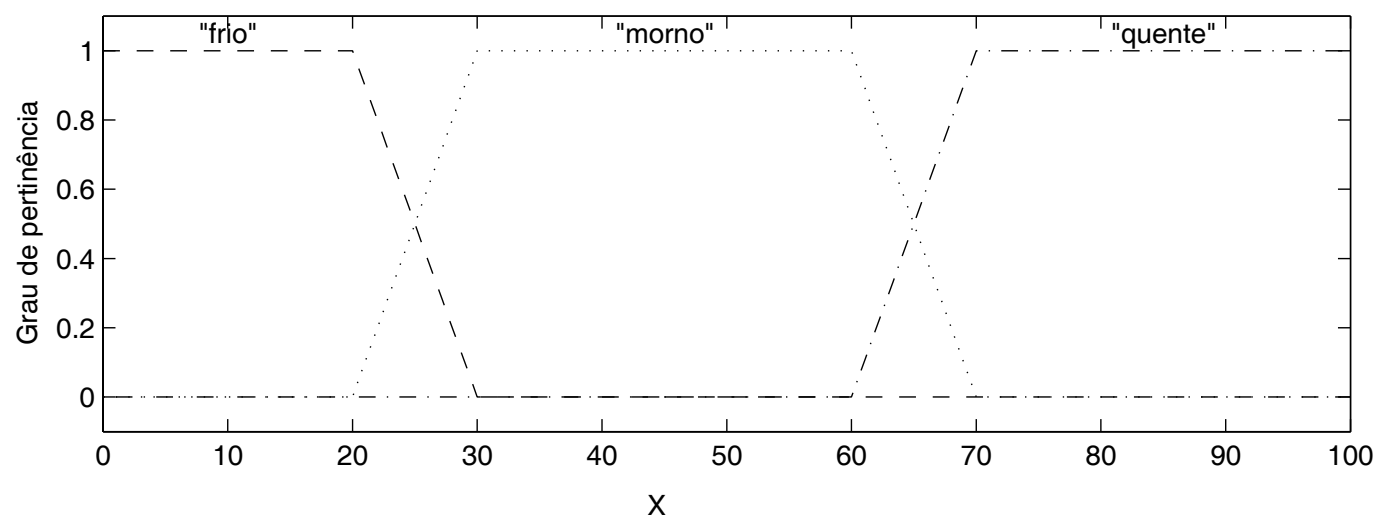

Figura 2.3: Exemplos de funções de pertinência dos conjunto fuzzy das temperaturas de um objeto.

A escolha das funções de pertinência está relacionada ao conhecimento de um sistema ou processo, por este motivo, estas podem ser expressas por uma função numérica ou tabulada que atribui valores discretos de uma variável, em seu universo de discurso. As funções de pertinência mais comuns na prática são as triangulares e as trapezoidais, pois podem ser implementadas com facilidade. Em casos onde é desejável que as funções de pertinência tenham transições mais suaves, pode-se usar funções gaussianas, sigmoidais, dentre outras, desde que estejam definidas no intervalo $[0,1]$. Na prática, uma boa interpretação das funções de pertinência de conjuntos fuzzy é que quanto maior o valor da pertinência para um elemento do conjunto, maior será a chance deste elemento ser um membro deste conjunto. Esta interpretação ajuda muito no entendimento de situações onde as fronteiras ou limites dos conjuntos fuzzy se interceptam, formando regiões ambíguas.

Um exemplo da descrição de conjuntos fuzzy via variáveis lingüísticas pode ser dado em termos da temperatura de um objeto, que pode variar na faixa de $0^{\circ}$ a $100^{\circ} \mathrm{C}$ $(\mathbb{X} \in \mathbb{R}$ e $\mathbb{S} \subset \mathbb{X}: \mathbb{S}=\{x \in \mathbb{R} \mid 0 \leq x \leq 100\}$, onde $x$ é a temperatura do objeto). Nestas condições, denomina-se "frio" o conjunto para temperaturas entre $0^{\circ}$ a $20^{\circ} \mathrm{C}$, "morno" o conjunto para temperaturas entre $20^{\circ}$ a $60^{\circ} \mathrm{C}$ e "quente" o conjunto para temperaturas entre $60^{\circ}$ a $100^{\circ} \mathrm{C}$. Desta forma, os adjetivos "frio", "morno" e "quente" são variáveis lingüísticas dos conjuntos que relacionam as temperaturas do objeto. A Figura 2.3 apresenta exemplos de funções de pertinência para os conjuntos "frio", "morno" e "quente".

Note que os conjuntos ordinários são casos especiais dos conjuntos fuzzy, visto que seus graus de pertinência somente assumem valores 0 ou 1 , é o que se chama de tran- 


\begin{tabular}{|c|c|c|}
\hline A & B & A e B \\
\hline $\mathbf{0}$ & $\mathbf{0}$ & $\mathbf{0}$ \\
\hline $\mathbf{0}$ & $\mathbf{1}$ & $\mathbf{0}$ \\
\hline $\mathbf{1}$ & $\mathbf{0}$ & $\mathbf{0}$ \\
\hline $\mathbf{1}$ & $\mathbf{1}$ & $\mathbf{1}$ \\
\hline \multicolumn{3}{|c|}{ E } \\
\hline \multicolumn{1}{|c|}{} \\
\hline
\end{tabular}

\begin{tabular}{|l|l|c|}
\hline A & B & A ou B \\
\hline $\mathbf{0}$ & $\mathbf{0}$ & $\mathbf{0}$ \\
\hline $\mathbf{0}$ & $\mathbf{1}$ & $\mathbf{1}$ \\
\hline $\mathbf{1}$ & $\mathbf{0}$ & $\mathbf{1}$ \\
\hline $\mathbf{1}$ & $\mathbf{1}$ & $\mathbf{1}$ \\
\hline \multicolumn{3}{|c|}{ OU } \\
\hline \multicolumn{3}{|c|}{} \\
\hline
\end{tabular}

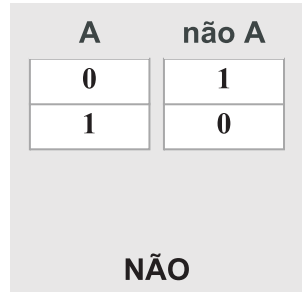

Figura 2.4: Resumo das operações da lógica Booleana padrão.

sição abrupta (indicando que um elemento do conjunto pertence ou não a determinado domínio). Logo, conjuntos fuzzy que não são conjuntos ordinários, são denominados apenas de conjuntos fuzzy, ou seja, conjuntos cujos graus de pertinência apresentam transições graduais, não assumindo apenas os valores 0 ou 1 . Mais detalhes sobre conjuntos fuzzy podem ser encontrados em Shaw e oes (1999) ou em Wang (1997).

\subsubsection{Operações lógicas}

As operações envolvendo conjuntos fuzzy são realizadas utilizando um superconjunto de lógica Booleana padrão, ou seja, um conjunto de operações de lógica Booleana aumentado. As operações envolvendo a lógica Booleana padrão são efetuadas através dos operadores E, OU e NÃO, as quais são resumidas na Figura 2.4. Além destes operadores, na lógica fuzzy existem outros operadores para efetuar as operações lógicas que são basicamente: os operadores das normas triangulares (normas-t) e os operadores das normas duais (normas-s ou ainda co-normas-t). Desta forma, caso seja mantido os valores de pertinência nos extremos do conjunto fuzzy, isto é, 1 (completamente verdadeiro) e 0 (completamente falso), as operações lógicas E, OU e NÃO são asseguradas. No entanto, além destas operações, o projetista de controle ainda dispõe de outras operações para especificar as implicações ou regras fuzzy para o sistema de controle. Detalhes sobre as operações envolvendo conjuntos fuzzy podem ser encontrados em Shaw e oes (1999) ou em Wang (1997).

\subsubsection{Inferência fuzzy}

A lógica fuzzy tem por objetivo fazer um mapeamento de um espaço de entrada para um espaço de saída, tendo como mecanismo primário uma lista de declarações do tipo 


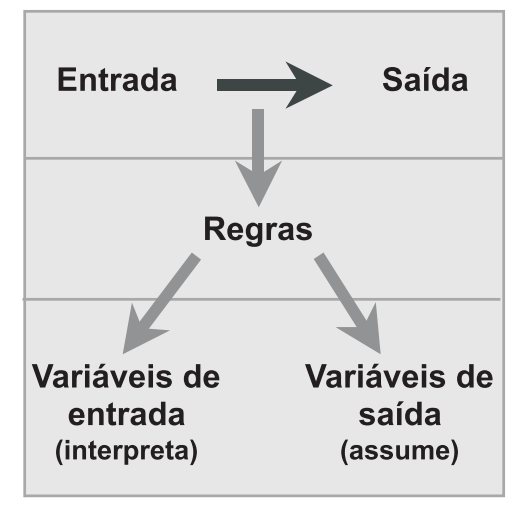

Figura 2.5: Diagrama de um processo de inferência fuzzy.

SE-ENTÃO chamadas regras de inferência. Todas as regras são avaliadas em paralelo, ou simultaneamente, não sendo por este fato importante a imposição de ordem entre estas. Em geral, as regras se referem às variáveis e aos adjetivos que as descrevem, por isso o processo de inferência fuzzy deve ser construído para a interpretação destas regras com base nas variáveis envolvidas. Na Figura 2.5 é apresentado um diagrama de um processo de inferência fuzzy padrão. Detalhes sobre o processo de inferência fuzzy podem ser encontrados em Shaw e oes (1999) ou em Wang (1997).

\subsubsection{Controladores fuzzy}

Os controladores fuzzy combinam informações qualitativas e quantitativas da operação de um sistema real através de alguma hierarquia. Existem basicamente dois tipos de controladores fuzzy:

- o controlador fuzzy Mamdani (Mamdani e Assilan, 1975);

- o controlador fuzzy Takagi-Sugeno (TS) (Takagi e Sugeno, 1985).

Ambos tipos de controladores têm como característica básica a flexibilidade na solução de controle, visto que utilizam informações da operação do sistema em sua estrutura, porém diferem entre si no procedimento de projeto. O controlador fuzzy Mamdani é um controlador empírico, cujo projeto segue um procedimento de ajuste heurístico baseado no conhecimento humano sobre a operação do sistema. O controlador fuzzy TS, além de contar com o conhecimento humano sobre a operação do sistema, segue 


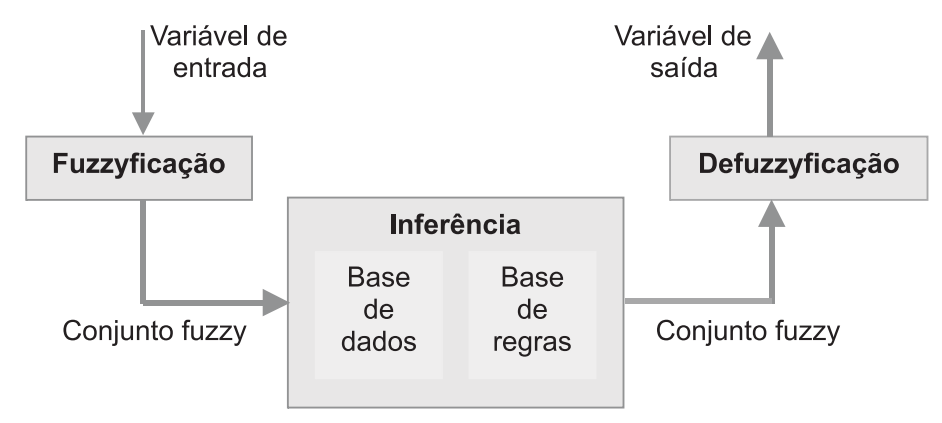

Figura 2.6: Diagrama em blocos de um controlador fuzzy.

um procedimento de ajuste sistemático baseado na modelagem fuzzy e nas teorias de controle não-linear e robusto já existentes.

A estrutura básica de um controlador fuzzy é apresentada na Figura 2.6. A assim chamada 'fuzzyficação' consiste no processo de transformação das variáveis definidas no domínio dos números reais, que é o domínio das variáveis do sistema, para o domínio dos números fuzzy, que é o domínio onde são realizadas as operações envolvendo lógica fuzzy. Para 'fuzzyficar' uma variável é necessário que se estabeleça uma relação entre os valores numéricos associados aos termos lingüísticos de conjuntos fuzzy. Esta relação é dada através de funções de pertinência. A base de conhecimento e a inferência são usadas nas tomadas de decisões de controle. A base de conhecimento é constituída por uma base de dados e uma base de regras fuzzy lingüísticas. A base de dados relaciona os valores numéricos associados às funções de pertinência. A base de regras caracteriza os objetivos e a estratégia de controle a ser utilizada, além de conter todas as possíveis combinações das ações de controle relativas às entradas e saídas do sistema. A inferência usa as operações envolvendo lógica fuzzy para simular tomadas de decisões humanas. Finalmente, a 'defuzzyficação' tem o objetivo de realizar transformações de ações de controle fuzzy inferidas em ações de controle reais.

Os controladores fuzzy Mamdani podem estimar variáveis de entrada e saída através de procedimento de ajuste heurístico, onde uma variável de entrada aciona uma inferência fuzzy que por conseguinte gera uma variável de saída. Todas as possíveis decisões que um controlador fuzzy Mamdani pode tomar são definidas a partir do conhecimento da operação do sistema e dispostas em uma tabela, chamada tabela de regras. A inferência fuzzy lingüística é geralmente realizada aplicando a operação de max-min (Shaw e oes, 1999) para 'defuzzyficação', porém existem outros métodos de 'defuzzyficação' 
disponíveis e que podem ser adotados, tais como o critério do valor máximo, as médias entre os valores máximos e o centro de área, etc. Sendo este último método o mais comum, por ser contínuo (Shaw e oes, 1999).

Especificamente para o caso de controladores fuzzy Mamdani, a etapa de projeto não necessita de um modelo explícito do sistema, somente do conhecimento de como o sistema se comporta frente às ações de controle manuais, que fica descrita em termos de regras fuzzy. Como já mencionado, estes controladores são sistemas empíricos, onde um conjunto de regras fuzzy é acionado como um mecanismo de decisão para ajuste dos efeitos causados por certas variações na operação do sistema. A ausência de um modelo explícito torna mais fácil a tarefa do projetista e permite a produção de controladores satisfatórios, com o mínimo de esforço. No entanto, esta ausência de modelo causa um certo ceticismo a respeito deste tipo de controlador, no sentido de que tanto a dinâmica do sistema quanto as tarefas de controle são descritas por regras, o que aparentemente é uma estratégia de alto nível. Logo, é necessário a inclusão de um controlador convencional, considerado de baixo nível, para desempenhar a tarefa de uma regulação mais detalhada, visando a confiabilidade da operação. Em adição, a ausência de um modelo torna muito difícil a formulação de provas e demonstrações, estas importantes principalmente quando é necessária uma operação mais confiável. Em virtude disso, foram introduzidas modelagens matemáticas para os controladores fuzzy. O uso de modelagem fuzzy se justifica pelo fato de que é possível formular condições que garantam o desempenho dos controladores fuzzy expressando tanto o sistema quanto o controlador no domínio fuzzy. Esta é a idéia principal do controlador fuzzy TS, que será abordado com mais particularidades no que segue.

\subsection{Modelagem e Controle Fuzzy TS}

A idéia básica da modelagem de sistemas fuzzy TS consiste na descrição aproximada de um sistema não-linear como uma agregação fuzzy ou interpolação de um certo número de modelos lineares invariantes no tempo locais ou subsistemas, que descrevem aproximadamente o comportamento do sistema em diferentes pontos ou regiões do seu espaço de estado. Esta modelagem permite que o projetista utilize o seu conhecimento sobre o sistema para definir o número de subsistemas e os pontos nos quais estes devem operar. O modelo fuzzy resultante é então obtido através de uma interpolação dos subsistemas. 


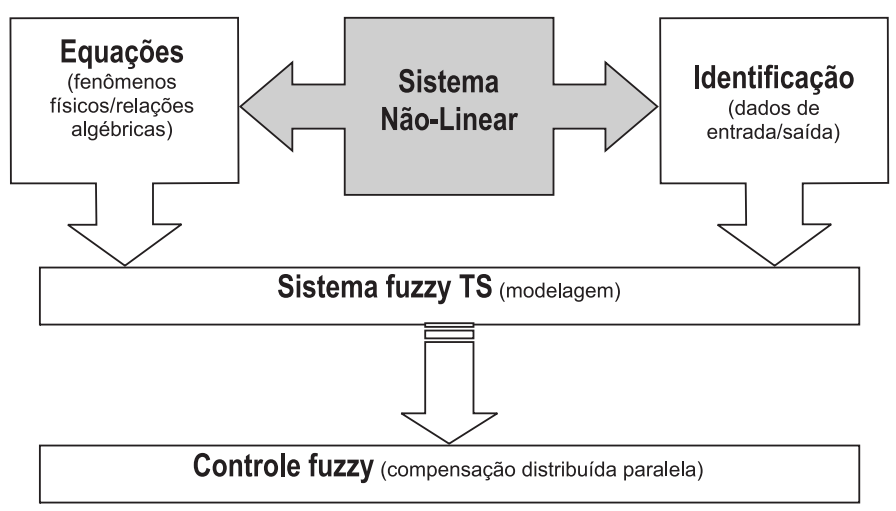

Figura 2.7: Esquema de projeto de um controlador baseado em sistemas fuzzy TS.

Desta forma, usando o assim chamado conceito de compensação distribuída paralela (CDP), pode-se projetar um controlador para cada subsistema e obter uma uma lei de controle fuzzy global, em geral não-linear, que também é uma agregação fuzzy dos controladores projetados individualmente. De fato, pode ser provado que tanto os sistemas quanto os controlares fuzzy TS são aproximadores universais (Tanaka e Wang, 2001). A Figura 2.7 apresenta um diagrama em blocos que ilustra o esquema de projeto de controladores fuzzy baseado em sistemas fuzzy TS.

Usualmente, o projeto de controle fuzzy TS é elaborado usando o conceito de estabilidade quadrática de sistemas lineares e funções de Lyapunov, o que resulta em um procedimento de busca de uma solução que satisfaça um conjunto de restrições expressas em termos das matrizes das representações lineares locais e das matrizes dos controladores. Sendo, portanto, um procedimento difícil se não impossível de ser efetuado sem auxílio de ferramentas computacionais dependendo do número de subsistemas adotado. Sendo assim, o projeto de controle fuzzy TS geralmente é formulado via LMI's, ou seja, as restrições do projeto são convertidas em LMI's, que podem ser implementadas, por exemplo, no módulo de controle LMI do Matlab (Gahinet et al., 1995). Exemplos de técnicas de controle fuzzy usando LMI's podem ser encontrados em Tanaka e Wang (2001), Li et al. (2000), Hong e Langari (2000), Hong e Langari (1998), Jadbabaie et al. (1998), Tanaka et al. (1998) e Wang et al. (1996). No que segue, apresenta-se uma breve introdução a LMI's utilizadas ao longo de todo o trabalho (Boyd et al., 1994).

Notação $1 \mathbb{R}^{n}$ and $\mathbb{R}^{n \times m}$ denotam o espaço Euclidiano $n$-dimensional e o conjunto de todas as matrizes reais de ordem $n \times m$, respectivamente, o sobrescrito " $T$ " denota a 
matriz transposta, $M>(\geq,<, \leq) 0$ denota uma matriz positiva (semi-positiva, negativa, semi-negativa) definida e $\sum_{i<j}^{R}$ indica, por exemplo para $R=3, \sum_{i<j}^{3} a_{i j} \Leftrightarrow a_{12}+a_{13}+$ $a_{23}$.

Definição 2 Uma LMI é uma desigualdade matricial da forma

$$
F(x)=F_{0}+\sum_{i=1}^{m} x_{i} F_{i}>0
$$

onde $x=\left[x_{1}, x_{2}, \ldots, x_{m}\right]^{T}$ é a variável e $F_{i}=F_{i}^{T}, i=1,2, \ldots, m$ são matrizes conhecidas.

A LMI (2.5) é uma restrição na variável $x$, em que o conjunto $\{x \mid F(x)>0\}$ é convexo. Um problema LMI pode ser formulado como segue.

Problema 1 Dada uma LMI $F(x)>0$, encontrar $x^{\text {fac }}$, tal que $F\left(x^{f a c}\right)>0$ ou determinar que a LMI é infactivel. Este é um problema de factibilidade convexo.

Lema 1 Sejam $Q(x)=Q(x)^{T}, R(x)=R(x)^{T}$ e $S(x)$ matrizes de dimensões apropriadas com dependência afim de $x$. A LMI

$$
\left[\begin{array}{ll}
Q(x) & S(x) \\
S(x)^{T} & R(x)
\end{array}\right]>0
$$

é equivalente às seguintes desigualdades

$$
\begin{aligned}
& R(x)>0, Q(x)-S(x) R(x)^{-1} S(x)^{T}>0 \\
& Q(x)>0, R(x)-S(x)^{T} Q(x)^{-1} S(x)>0
\end{aligned}
$$

As desigualdades (2.7) e (2.8) são chamadas complementos de Schur.

\subsubsection{Modelagem fuzzy TS}

Considere a classe de sistemas não-lineares descrita na forma espaço de estado

$$
\dot{x}=f(x)+g(x) u ; x(0)=x_{0}
$$


onde $x \in \mathbb{R}^{n}, u \in \mathbb{R}^{m}$ e $x_{0}$ são os vetores de variáveis de estado, de variáveis de entrada e de condições iniciais do sistema, respectivamente. No que segue, considera-se a existência de um equilibro $(x, u)=0$, isto é, $f(0)=0$ e $g(0)=0$ e que as funções $f(\cdot), g(\cdot) \in C_{1}^{\rho+1}$, com $\rho>1$, que significa que $f(\cdot)$ e $g(\cdot)$ têm a $(\rho+1)$-ésima derivada em relação a $x$ definidas em um conjunto compacto $\mathbb{X} \subset \mathbb{R}^{n}$.

O sistema fuzzy TS é descrito por regras fuzzy do tipo SE-ENTÃO, que representam relações entrada-saída lineares locais do sistema não-linear (2.9), cuja $i$-ésima regra é dada por

$$
\begin{array}{ll}
\text { Regra } i: & \text { SE } \tilde{z}_{1} \text { é } N_{i 1} \text { e } \tilde{z}_{2} \text { é } N_{i 2} \text { e } \ldots \text { e } \tilde{z}_{p} \text { é } N_{i p} \\
& \text { ENTÃO } \dot{x}=A_{i} x+B_{i} u \\
& i=1,2, \ldots, R
\end{array}
$$

onde $R$ é o número de regras de inferência, $A_{i} \in \mathbb{R}^{n \times n}$ e $B_{i} \in \mathbb{R}^{n \times m}$ são matrizes das representações lineares locais de $f(\cdot)$ e $g(\cdot)$ em relação a $x$, respectivamente, $N_{i j}$ é o $j$-ésimo conjunto fuzzy da regra $i$ e $\tilde{z} \in \mathbb{R}^{p}$ é o vetor de variáveis premissas. Em geral, as variáveis premissas podem ser funções das variáveis de estado e de distúrbios externos e/ou tempo (Li et al., 2000). Usualmente, considera-se que as variáveis premissas não dependem das variáveis de entrada $u$. Esta consideração é utilizada para evitar um processo de 'defuzzyficação' complicado para os controladores fuzzy (Tanaka et al., 1998). A parte SE do sistema fuzzy (2.10) é denominada de parte antecedente e a parte ENTÃO é denominada de parte conseqüente. O termo " $A_{i} x+B_{i} u$ " na parte conseqüente é referenciado como subsistema.

Desta forma, dado o par $(x, u)$, o sistema fuzzy TS global ou resultante é inferido como a média ponderada de todos os subsistemas, que resulta em

$$
\dot{x}=\sum_{i=1}^{R} n_{i}(\tilde{z})\left(A_{i} x+B_{i} u\right)
$$

onde

$$
n_{i}(\tilde{z})=\frac{\prod_{k=1}^{p} N_{i k}\left(\tilde{z}_{k}\right)}{\sum_{j=1}^{R} \prod_{k=1}^{p} N_{j k}\left(\tilde{z}_{k}\right)}
$$

denota a função de pertinência normalizada, $\operatorname{com} N_{i k}\left(\tilde{z}_{k}\right) \in[0,1]$ o grau de pertinência 
de $\tilde{z}_{k}$ no conjunto fuzzy $N_{i k}$. Considerando o fato de que em $(2.12), N_{i k}\left(\tilde{z}_{k}\right) \geq 0$, $i=1,2, \ldots, R$, tem-se

$$
n_{i}(\tilde{z}) \geq 0 ; i=1,2, \ldots, R
$$

$\mathrm{e}$

$$
\sum_{i=1}^{R} n_{i}(\tilde{z})=1
$$

Em geral, um sistema fuzzy TS pode aproximar qualquer função não-linear suave $F$ definida em uma região compacta $\mathbb{X} \subset \mathbb{R}^{n}$, diante das seguintes considerações: (i) $F(0)=0$ e (ii) $F \in C_{1}^{2}$. Assim sendo, $F, \partial F / \partial x, \partial^{2} F / \partial x^{2}$ são contínuas e limitadas em $\mathbb{X}$. Mais detalhes da teoria de sistemas fuzzy TS como aproximadores universais podem ser obtidos em Tanaka e Wang (2001).

\subsubsection{Construção do modelo fuzzy}

Em geral, existem duas abordagens para construção de sistemas fuzzy TS:

- identificação usando dados de entrada-saída de um sistema;

- equações do sistema não-linear.

O processo de identificação usando dados de entrada-saída de um sistema pode ser dividido em duas etapas: (1) estrutural, onde é arbitrada uma certa estrutura (ou modelo) para o método de identificação e (2) obtenção dos parâmetros da estrutura arbitrada. Esta aproximação é adequada para plantas cuja dinâmica é difícil ou até impossível de ser representada por equações analíticas e ou dinâmicas. Entretanto, muitos sistemas dinâmicos não-lineares podem ser caracterizados por equações dinâmicas e ou analíticas, portanto, a segunda abordagem é mais apropriada (Tanaka e Wang, 2001). A construção de sistemas fuzzy a partir de equações que representam a dinâmica do sistema não-linear é a abordagem usada ao longo deste trabalho.

Existem duas aproximações disponíveis na literatura para obtenção de um sistema fuzzy:

- a aproximação setorial;

- a aproximação local. 
A aproximação setorial garante a construção de um sistema fuzzy exato a partir de setores globais, o que pode levar a um elevado número de regras. No entanto, muitas vezes, torna-se difícil encontrar setores globais associados a certos sistemas não-lineares. Nestes casos, setores locais podem ser considerados e a aproximação obtida pode ser razoável quando as variáveis do sistema físico forem limitadas em faixas ou regiões, o que pode reduzir consideravelmente o número de regras do sistema fuzzy (Tanaka e Wang, 2001). No contexto da aproximação local de sistemas não-lineares, existe uma fórmula de linearização que produz uma boa aproximação do sistema não-linear na vizinhança de um ponto de operação escolhido para linearização, mesmo que este ponto de operação não seja um ponto de equilíbrio (Teixeira e Żak, 1999). A seguir, descrevese o procedimento de obtenção de representações lineares usando a fórmula de Teixeira \& Żak.

\section{Obtenção das representações lineares}

Considere o sistema não-linear definido em (2.9) e $\bar{x}$ um ponto de linearização, não necessariamente um ponto de equilíbrio. O objetivo é obter matrizes $A$ e $B$ de dimensões apropriadas, tais que na vizinhança de $\bar{x}$ o sistema (2.9) possa ser descrito por

$$
f(x)+g(x) u \approx A x+B u
$$

e

$$
f(\bar{x})+g(\bar{x}) u \approx A \bar{x}+B u
$$

para $x, u, f(\cdot)$ e $g(\cdot)$ como já definidos. Dado que $u$ é arbitrário, tem-se $g(\bar{x})=B$. Desta forma o procedimento se reduz a encontrar a matriz $A$, tal que na vizinhança de $\bar{x}$

$$
f(x) \approx A x
$$

e

$$
f(\bar{x}) \approx A \bar{x}
$$


A fórmula de linearização de Teixeira \& Żak

$$
a_{i}=\nabla f_{i}(\bar{x})+\frac{f_{i}(\bar{x})-\bar{x}^{T} \nabla f_{i}(\bar{x})}{\|\bar{x}\|^{2}} \bar{x} ; \bar{x} \neq 0
$$

é obtida da solução de um problema de otimização (veja Teixeira e Żak (1999)), com $a_{i}$ as colunas da matriz $A$ e $\nabla f_{i}(\bar{x}): \mathbb{R}^{n} \rightarrow \mathbb{R}^{n}$ o gradiente, um vetor coluna de $f_{i}($. calculado em relação a $x$. Esta fórmula produz aproximações lineares em vez de afins, geralmente obtidas da fórmula de linearização de Taylor. Para verificar esta afirmação, considere a fórmula de linearização de Taylor

$$
A=\nabla f(\bar{x}):=\left.\frac{\partial f(x)}{\partial x}\right|_{x=\bar{x}}
$$

cuja aproximação de $f(\cdot)$ em torno de $\bar{x}$ é dada por

$$
f(x) \approx f(\bar{x})+\nabla f(\bar{x})(x-\bar{x}) .
$$

Desta forma, quando $f(\bar{x}) \neq 0$, ou seja, quando $\bar{x}$ não é ponto de equilíbrio, esta aproximação produz modelos afins em vez de lineares, como mencionado. Utilizando a fórmula de linearização de Teixeira \& Żak, pode-se obter várias representações lineares de um sistema não-linear em pontos de linearização escolhidos e assim construir uma representação fuzzy deste sistema.

Observação 2 A técnica de aproximação local leva à redução do número de regras de inferência para os sistemas fuzzy. Entretanto, a lei de controle baseada no sistema fuzzy aproximado pode não garantir a estabilidade do sistema não-linear original sob tal lei de controle. Uma das abordagens utilizadas para amenizar este problema é a introdução de projetos de controle fuzzy robusto (Tanaka e Wang, 2001).

\subsubsection{Controle fuzzy TS}

O projeto de controle fuzzy TS está baseado no conceito de CDP, que consiste no projeto distributivo de controladores locais para a compensação dos subsistemas que compõem o sistema fuzzy TS. O controlador resultante é uma interpolação dos controladores locais, sendo, portanto, um controlador não-linear. A seguir, detalha-se o procedimento 


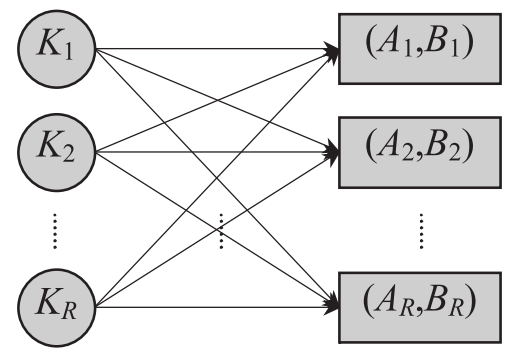

Figura 2.8: Compensação distribuída paralela.

da CDP para a obtenção do controlador fuzzy TS com realimentação de estado. A Figura 2.8 apresenta um diagrama em blocos que ilustra o conceito de CDP.

Usando a idéia do controle com realimentação de estado e o conceito da CDP, um controle natural para o sistema fuzzy TS (2.10) compartilha a mesma estrutura de regras, isto é,

$$
\begin{array}{ll}
\text { Regra } i: & \text { SE } \tilde{z}_{1}(x) \text { é } N_{i 1} \text { e } \tilde{z}_{2}(x) \text { é } N_{i 2} \text { e } \ldots \text { e } \tilde{z}_{p}(x) \text { é } N_{i p} \\
& \text { ENTÃO } u=-K_{i} x \\
& i=1,2, \ldots, R
\end{array}
$$

onde $K_{i} \in \mathbb{R}^{m \times n}$ são os ganhos de realimentação de estado. Assim como o sistema fuzzy TS, o controlador fuzzy resultante também é uma média ponderada dos controladores locais, ou seja,

$$
u=-\sum_{i=1}^{R} n_{i}(\tilde{z}) K_{i} x
$$

com $n_{i}(\cdot)$ definido em (2.12). Substituindo (2.23) em (2.11), obtém-se

$$
\dot{x}=\sum_{i=1}^{R} \sum_{j=1}^{R} n_{i}(\tilde{z}) n_{j}(\tilde{z})\left(A_{i}-B_{i} K_{j}\right) x .
$$

Usando o fato de que

$$
\sum_{i=1}^{R} \sum_{j=1}^{R} n_{i}(\tilde{z}) n_{j}(\tilde{z})=\sum_{i=1}^{R} n_{i}^{2}(\tilde{z})+2 \sum_{i<j}^{R} n_{i}(\tilde{z}) n_{j}(\tilde{z})
$$


pode-se reescrever a equação (2.24) da seguinte forma

$$
\dot{x}=\sum_{i=1}^{R} n_{i}^{2}(\tilde{z}) G_{i i} x+2 \sum_{i<j}^{R} n_{i}(\tilde{z}) n_{j}(\tilde{z})\left[\frac{G_{i j}+G_{j i}}{2}\right] x
$$

onde $G_{i j}:=A_{i}-B_{i} K_{j}$. A equação (2.25) é útil para o projeto de controle fuzzy TS, visto que reduz a quantidade de restrições a serem avaliadas na fase de projeto.

O projeto do controlador fuzzy TS consiste na obtenção dos ganhos de realimentação de estado $K_{i}, i=1,2, \ldots, R$. Usando a CDP, tem-se um procedimento simples e natural para obtenção de um sistema de controle não-linear, enquanto que outras técnicas de controle não-linear necessitam de conhecimentos mais elaborados e especiais (Tanaka e Wang, 2001). Embora o controlador fuzzy TS seja construído usando uma estrutura de projeto local, os ganhos de realimentação de estado devem ser determinados usando condições de projeto globais, que são necessárias para garantir a estabilidade global e, portanto, o desempenho do controlador em malha-fechada. Geralmente, a obtenção destes ganhos é elaborada usando o conceito de estabilidade quadrática e uma função de Lyapunov do tipo $V(x)=x^{T} P x, P=P^{T}, P>0$, que envolve a busca de uma matriz simétrica positiva definida comum $P$ como solução de um conjunto de restrições. No contexto de LMI's, apresenta-se o seguinte projeto básico de controle fuzzy TS estabilizante proposto por Tanaka et al. (1998).

Teorema 2 Se existe uma matriz $X=X^{T}, X>0$ e matrizes $Y_{i}, i=1,2, \ldots, R$ de dimensões apropriadas satisfazendo as seguintes LMI's

$$
\begin{aligned}
X=X^{T} & >0 \\
X A_{i}^{T}+A_{i} X-Y_{i}^{T} B_{i}^{T}-B_{i} Y_{i} & <0 ; \forall i=1,2, \ldots, R \\
X A_{i}^{T}+A_{i} X-Y_{j}^{T} B_{i}^{T}-B_{i} Y_{j} & \\
+X A_{j}^{T}+A_{j} X-Y_{i}^{T} B_{j}^{T}-B_{j} Y_{i} & <0 ; \forall i<j ; i, j=1,2, \ldots, R
\end{aligned}
$$

onde $X=P^{-1}$ e $Y_{i}=K_{i} X$, então o sistema fuzzy TS com realimentação de estado (2.25) é globalmente assintoticamente estável no equilíbrio $x=0$.

Desta forma, se as LMI's (2.26)-(2.28) forem satisfeitas, os ganhos de realimentação de estado do controlador fuzzy TS (2.23) podem ser obtidos como $K_{i}=Y_{i} X^{-1}, i=$ 
$1,2, \ldots, R$.

O projeto de controle usando as LMI's apresentadas no Teorema 2 pode não produzir um resultado factível para sistemas fuzzy TS constituídos por um elevado número de regras. Neste caso, pode-se flexibilizar ou relaxar o projeto de controle fuzzy TS explorando as propriedades (2.13) e (2.14) das funções de pertinência normalizadas. Exemplos deste tipo de projeto podem ser encontrados em Teixeira et al. (2003), Teixeira et al. (2000) e Tanaka et al. (1998). Ainda no contexto de flexibilização do projeto de controle fuzzy TS, visando reduzir o conservadorismo na busca de uma solução factível,

uma função de Lyapunov fuzzy do tipo $V(x)=\sum_{i=1}^{R} n_{i}(\tilde{z}) x^{T} P_{i} x, \operatorname{com} P_{i}=P_{i}^{T}, P_{i}>0$ pode ser considerada, o que resulta em novas condições de estabilidade para os sistemas fuzzy TS e, conseqüentemente, para o projeto de controle. Este tipo de abordagem pode ser encontrado em Tanaka et al. (2003). Além destas, ainda existem outras abordagens de projeto de controle fuzzy TS utilizando a teoria de controle robusto, que vêm sendo aplicadas com sucesso na estabilização de sistemas não-lineares com incertezas na modelagem e distúrbios externos desconhecidos. Exemplos de técnicas de controle fuzzy TS robusto podem ser encontrados em Arrifano e Oliveira (n.d.a), Hong e Langari (2000) e Tanaka et al. (1996).

\subsection{Modelagem e Controle Fuzzy com Saltos Markovianos}

Muitos sistemas dinâmicos podem apresentar mudanças abruptas e aleatórias quando em funcionamento em virtude de mudanças em seus parâmetros ou até mesmo em sua estrutura, que podem ser causadas por falhas ou reparos de seus componentes, de suas interconexões com os subsistemas e até por distúrbios decorrentes de alterações ambientais. Os sistemas com estas características podem ser modelados como operando em diferentes modos, onde cada modo corresponde a alguma combinação destes eventos e são referenciados na literatura como sistemas com saltos Markovianos, seguindo a teoria dos processos de Markov.

A classe de sistemas lineares com saltos Markovianos (SLSM) vem sendo extensivamente estudada para representação de diversos tipos de sistemas dinâmicos, tais como, sistemas de manufatura, sistemas de potência, telecomunicações, dentre outros (Mariton, 1990). Esta diversidade de aplicações gerou grande interesse nesta classe 
de sistemas, originando diversos resultados, tais como, estabilidade, estabilização, observabilidade, controlabilidade, robustez, dentre outros. Particularmente, em aplicações envolvendo a estabilização de SLSM, tanto incertezas na modelagem, quanto rejeição de distúrbios externos vêm sendo consideradas. Usualmente, as incertezas consideradas aparecem na forma estruturada e limitadas por norma, facilitando a adaptação das técnicas de controle robusto do caso determinístico para esta classe de sistemas. Exemplos destas técnicas podem ser encontrados em Boukas et al. (2003), Boukas et al. (1999), Boukas e Yang (1999a) e Costa e Boukas (1998).

Sabe-se que uma representação mais precisa dos sistemas com saltos Markovianos deve considerar as dinâmicas não-lineares, visando caracterizar melhor os sistemas reais. Por este motivo, a representação linear em cada modo de operação pode dificultar o controle, visto que, ele somente atuará em uma região próxima ao ponto de operação escolhido, daí a preocupação em considerar incertezas nos parâmetros, com o intuito de ampliar a faixa de operação dos controladores. Como antes mencionado, ao que parece, apenas um trabalho considerando o problema de controle ótimo para uma classe de SNLSM foi registrado. Sendo assim, introduz-se a seguir uma classe de SNLSM a partir da qual é proposta uma representação fuzzy para aproximação de suas dinâmicas. Posteriormente, esta representação ou modelagem fuzzy é utilizada no projeto de controle fuzzy, assim como no caso da modelagem e controle fuzzy TS apresentados.

Notação $2 \Omega$ corresponde ao espaço amostral (conjunto de possiveis resultados de um experimento aleatório), $\mathcal{F}$ correponde a uma família de eventos $(\mathcal{F} \subset \Omega)$, Pr é uma medida de probabilidade definida em uma $\sigma$-álgebra $\mathcal{F},(\Omega, \mathcal{F}, \operatorname{Pr})$ é o espaço de probabilidade e $\mathcal{L}_{2}[0, T]$ denota o espaço de funções vetoriais quadráticas integráveis no intervalo $[0, T]$.

\subsubsection{Modelagem fuzzy com saltos Markovianos}

A classe de sistemas dinâmicos não-lineares considerada neste trabalho é uma classe de sistemas híbridos definida no produto de um espaço vetorial Euclidiano com um conjunto finito discreto. A parte definida no espaço vetorial Euclidiano consiste de um vetor de estado e de um vetor de entrada do sistema $x \in \mathbb{R}^{n}$ e $u \in \mathbb{R}^{m}$, respectivamente, e a parte definida em um conjunto finito discreto consiste de um processo de Markov no tempo contínuo $r$ que assume valores no espaço discreto $\mathbb{S}=\{1,2, \ldots, N\}$, determinando assim 


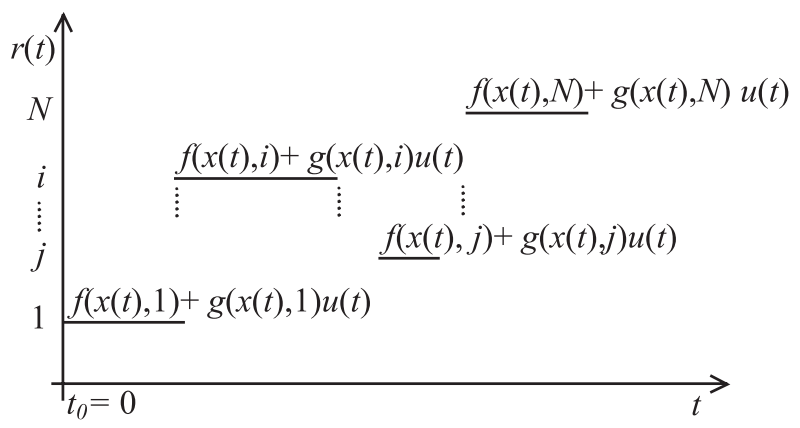

Figura 2.9: Evolução no tempo de um SNLSM seguindo as mudanças de modo do processo de Markov.

os modelos de regime ou modos do sistema.

Seja a classe de SNLSM definida em $((\Omega, \mathcal{F}, \operatorname{Pr}),[0, T])$ e descrita na forma espaço de estado

$$
\dot{x}=f(x, r)+g(x, r) u ; x(0)=x_{0} ; r(0)=r_{0}
$$

onde $f(\cdot, \cdot): \mathbb{R}^{n} \times \mathbb{S} \rightarrow \mathbb{R}^{n}$ e $g(\cdot, \cdot): \mathbb{R}^{n} \times \mathbb{S} \rightarrow \mathbb{R}^{m}, \operatorname{com} f(0, \cdot)=0$ e $g(0, \cdot)=0$ são funções não-lineares suaves em relação ao primeiro argumento, $x_{0} \in \mathbb{R}^{n}$ é o vetor de estado inicial e $r_{0} \in \mathbb{S}$ é o modo inicial em que o sistema se encontra. A evolução do processo estocástico $\{r, t \geq 0\}$ que determina os modos assumidos pelo sistema a cada instante de tempo $t$ é descrita pela seguinte probabilidade de transição de um modo $i$ para um modo $j$, para $i, j \in \mathbb{S}$

$$
\operatorname{Pr}\{r(t+\Delta)=j \mid r(t)=i\}:= \begin{cases}\pi_{i j} \Delta+o(\Delta), & i \neq j \\ 1-\pi_{i} \Delta+o(\Delta), & i=j\end{cases}
$$

onde $\Delta>0, \lim _{\Delta \rightarrow 0} o(\Delta) \Delta^{-1}=0$ e $\pi_{i j} \geq 0$ e $\pi_{i}:=-\pi_{i i}=\sum_{j=1, j \neq i}^{N} \pi_{i j}$. A matriz $\Pi:=\left[\pi_{i j}\right]_{i, j \in \mathbb{S}}$ é chamada matriz de taxas de transição. Desta forma, o sistema (2.29) (2.30) representa uma família de $N$ dinâmicas não-lineares descritas por $f(\cdot, \cdot)$ e $g(\cdot, \cdot)$, que são governadas por $r$. Neste contexto, a variável discreta $r$ é referenciada como processo de Markov de estados ou modos finitos e a solução do sistema (2.29) - (2.30) é então um processo estocástico $x\left(t, x_{0}, r_{0}\right)$ definido em $(\Omega, \mathcal{F}, \operatorname{Pr})$. A Figura 2.9 ilustra a evolução no tempo de um SNLSM de acordo com o processo de Markov $r$.

Visando considerar os saltos na modelagem fuzzy para obter uma representação mais precisa e completa das dinâmicas do sistema (2.29), apresenta-se a seguir o sistema fuzzy 
com saltos Markovianos (SFSM), que foi construído seguindo a idéia de um sistema fuzzy chaveado (SFC) (Tanaka et al., 2000). O SFC possui um nível superior de região para efetuar mudanças abruptas e um nível inferior de regras para fazer interpolações de subsistemas. Assim como o SFC, o SFSM é estruturado em um nível superior para efetuar as transições entre os modos assumidos pelo porcesso de Markov $r$ e um nível inferior para as regras fuzzy em cada modo, que descrevem as não-linearidades no vetor de estado $x$. Por analogia à abordagem dos sistemas fuzzy TS, a parte SE do SFSM é chamada de parte antecedente e a parte ENTÃO é chamada de parte conseqüente. Portanto, o $i$-ésimo modo do SNLSM é representado como segue

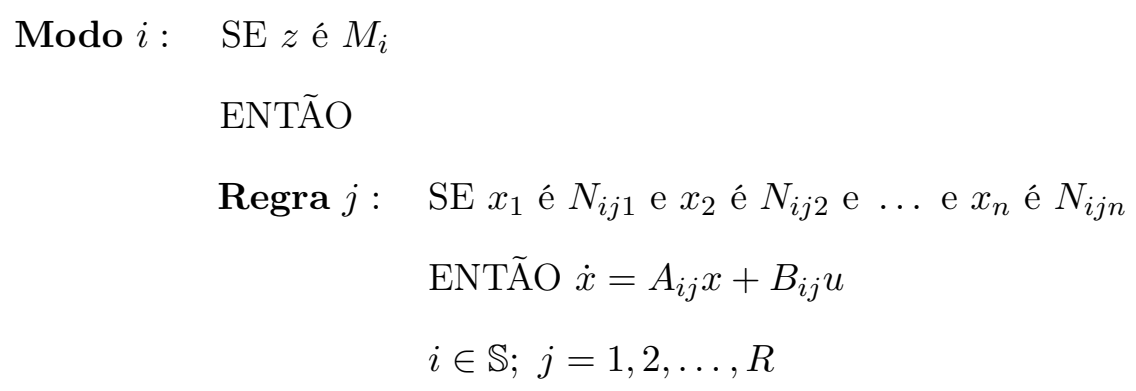

onde $z \in \mathbb{R}^{1}$ é uma variável indicadora de modo, $A_{i j}$ e $B_{i j}$ são matrizes de dimensões apropriadas, que descrevem as representações lineares locais do SNLSM em relação a $x$ na vizinhança de pontos de operação escolhidos, $M_{i}$ e $N_{i j}$ são conjuntos ordinário e fuzzy, respectivamente, e $R$ é o número de regras de inferência em cada modo do sistema.

Dado $(x, u, z)$, o SFSM resultante é inferido por uma interpolação dos modelos lineares locais $\left(A_{i j}, B_{i j}\right), i \in \mathbb{S}, j=1,2, \ldots, R$ como segue

$$
\dot{x}=\sum_{i=1}^{N} \sum_{j=1}^{R} m_{i}(z) n_{i j}(x)\left(A_{i j} x+B_{i j} u\right)
$$

onde $m_{i}(\cdot)$ é a função característica de $z$ no conjunto ordinário $M_{i}$ definida por

$$
m_{i}(z):= \begin{cases}1, & r=i \\ 0, & \text { caso contrário }\end{cases}
$$


e $n_{i j}(\cdot)$ é a função de pertinência normalizada do conjunto fuzzy $N_{i j k}$ definida por

$$
n_{i j}(x)=\frac{\prod_{k=1}^{n} N_{i j k}\left(x_{k}\right)}{\sum_{l=1}^{R} \prod_{k=1}^{n} N_{i l k}\left(x_{k}\right)}
$$

$\operatorname{com} N_{i j k}(x) \in[0,1]$ o grau de pertinência de $x_{k}, k=1,2, \ldots, n$ em $N_{i j k}$. Seguindo a modelagem fuzzy TS e considerando o fato de que em (2.34) $N_{i j k}\left(x_{k}\right) \geq 0$, tem-se

$$
n_{i j}(x) \geq 0 ; j=1,2, \ldots, R
$$

e

$$
\sum_{j=1}^{R} n_{i j}(x)=1 ; \forall i \in S
$$

O universo de discurso $\mathbb{X}: \mathbb{R}^{n} \times \mathbb{S} \rightarrow \mathbb{R}^{n}$ do SFSM é dado por

$$
\mathbb{X}=\bigcup_{i=1}^{N} \text { Modo } i=\text { Modo } 1 \cup \operatorname{Modo} 2 \cup \ldots \cup \operatorname{Modo} N
$$

Modo $i \cap$ Modo $\ell=\phi, i \neq \ell, i, \ell \in \mathbb{S}$.

Observação 3 A obtenção dos modelos lineares locais $\left(A_{i j}, B_{i j}\right), j=1,2, \ldots, R$, em cada modo $i \in \mathbb{S}$ pode ser feita através da fórmula de linearização de Teixeira $\&$ Żak apresentada na Seção 2.2.2.

Observação 4 A escolha das variáveis premissas z e $x$ do SFSM se justifica pelo fato de que na modelagem fuzzy as variáveis premissas podem ser tanto funções das variáveis de estado quanto de distúrbios externos e/ou tempo, como já mencionado. O uso de $z$ em vez de $r$ não significa que $r$ não está disponível para medição. A idéia é a de não limitar o modelo fuzzy ao uso de $r$, sendo que em sistemas reais é comum que $r$ seja definido a partir da medição de uma variável $z$, que, por exemplo, pode ser uma tensão elétrica (Arrifano e Oliveira, n.d.b), a velocidade do vento (Farias et al., 2000), etc.

\subsubsection{Controle fuzzy}

Tendo em vista o conceito de CDP, uma lei de controle natural para um sistema fuzzy compartilha a mesma estrutura de regras deste sistema. Desta forma, estendendo o 


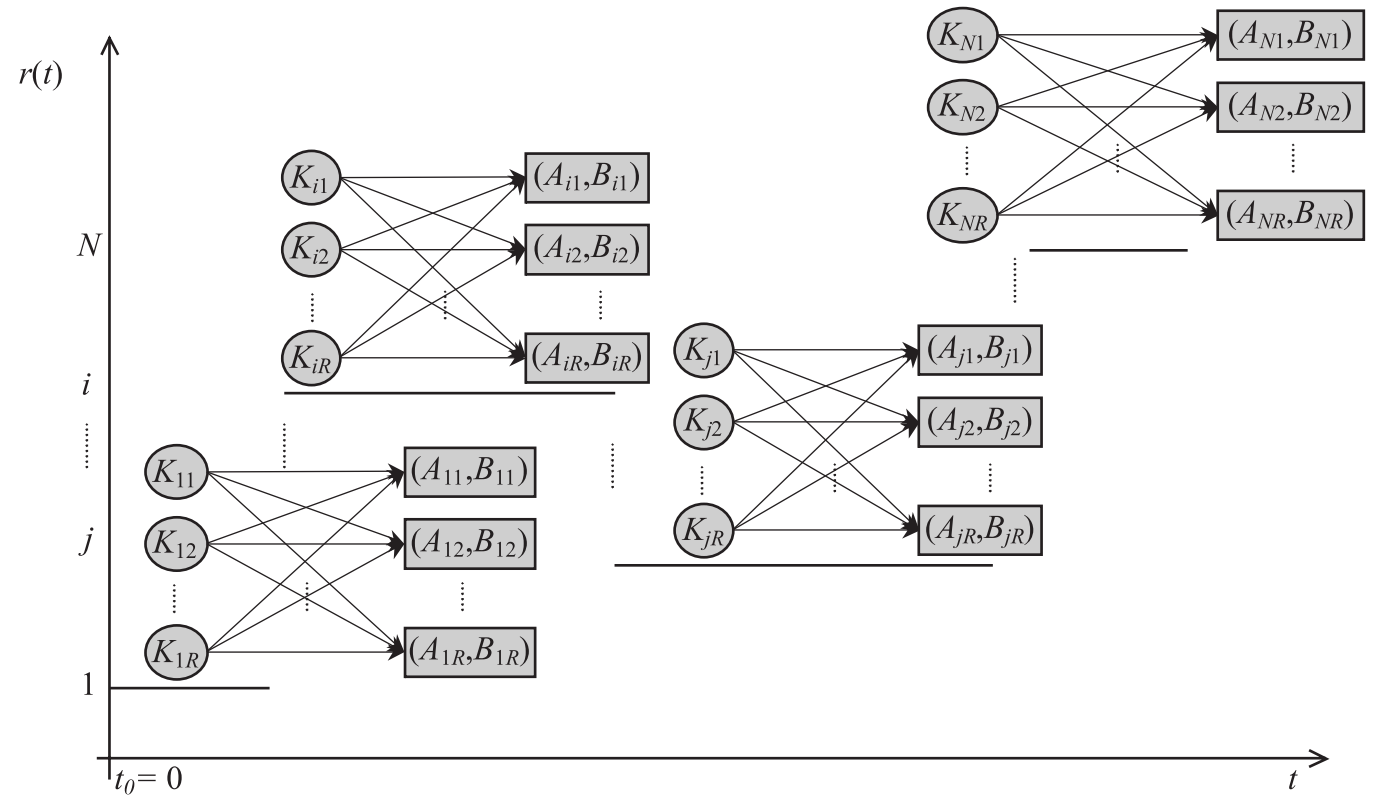

Figura 2.10: Esquema de CDP estendido para o controle do SFSM.

conceito de CDP, o controlador fuzzy para o $i$-ésimo modo do SFSM é dado por

$$
\begin{array}{ll}
\text { Modo } i: \quad & \text { SE } z \text { é } M_{i} \\
& \\
\text { ENTÃO } & \\
& \text { Regra } j: \quad \\
& \text { SE } x_{1} \text { é } N_{i j 1} \text { e } x_{2} \text { é } N_{i j 2} \text { e } \ldots \text { e } x_{n} \text { é } N_{i j n} \\
& \text { ENTÃO } u=-K_{i j} x \\
& i \in \mathbb{S} ; j=1,2, \ldots, R
\end{array}
$$

onde $K_{i j}$ são os ganhos de realimentação de estado de dimensões apropriadas. A Figura 2.10 ilustra o conceito de CDP usado no controle do SFSM.

Desta forma, o controlador fuzzy resultante é inferido por

$$
u=-\sum_{i=1}^{N} \sum_{j=1}^{R} m_{i}(z) n_{i j}(x) K_{i j} x
$$

com $m_{i}(\cdot)$ e $n_{i j}(\cdot)$ definidos em (2.33) e (2.34), respectivamente. Substituindo (2.38) 
em (2.32), resulta

$$
\dot{x}=\sum_{i=1}^{N} \sum_{j=1}^{R} m_{i}(z) n_{i j}(x)\left[A_{i j}-\left(\sum_{k=1}^{N} \sum_{l=1}^{R} m_{k}(z) n_{k l}(x) B_{i j} K_{k l}\right)\right] x .
$$

Tendo em vista que $m_{i}(z) m_{k}(z)=0, i \neq k, i, k \in \mathbb{S},(2.39)$ pode ser escrita na seguinte forma

$$
\dot{x}=\sum_{i=1}^{N} m_{i}(z)\left[\sum_{j=1}^{R} \sum_{k=1}^{R} n_{i j}(x) n_{i k}(x)\left(A_{i j}-B_{i j} K_{i k}\right)\right] x
$$

Agora, usando o fato de que em cada modo $i$

$$
\sum_{j=1}^{R} \sum_{k=1}^{R} n_{i j}(x) n_{i k}(x)=\sum_{j=1}^{R} n_{i j}^{2}(x)+2 \sum_{j<k}^{R} n_{i j}(x) n_{i k}(x)
$$

pode-se reescrever a equação (2.40) como

$$
\dot{x}=\sum_{i=1}^{N} m_{i}(z)\left[\sum_{j=1}^{R} n_{i j}^{2}(x) G_{i j}+2 \sum_{j<k}^{R} n_{i j}(x) n_{i k}(x) H_{i j k}\right] x
$$

$\operatorname{com} G_{i j}:=A_{i j}-B_{i j} K_{i j}$ e $H_{i j k}:=\frac{1}{2}\left(A_{i j}-B_{i j} K_{i k}+A_{i k}-B_{i k} K_{i j}\right), i \in \mathbb{S}, j, k=$ $1,2, \ldots, R$.

Observação 5 Supondo que o SFSM (2.41) no instante $t$ esteja no modo i, isto é, $r=i, i \in \mathbb{S}$, por (2.33) $m_{i}(z)=1$, tem-se

$$
\dot{x}=\sum_{j=1}^{R} n_{i j}^{2}(x) G_{i j}+2 \sum_{j<k}^{R} n_{i j}(x) n_{i k}(x) H_{i j k} x
$$

que recai na representação dos sistemas fuzzy TS com realimentação de estado (2.25).

Assim como no caso do projeto de controle fuzzy TS, o objetivo de projeto aqui é a obtenção dos ganhos de realimentação de estado $K_{i j}, i \in \mathbb{S}, j=1,2, \ldots, R$. Nos capítulos posteriores, apresentam-se técnicas de projeto usando a modelagem e controle fuzzy com saltos Markovianos. 


\section{Capítulo 3}

\section{Estabilidade e Estabilização}

\subsection{Estabilidade Estocástica}

O problema de estabilização de sistemas com saltos Markovianos vem sendo explorado utilizando conceitos de estabilidade de segundo momento, tais como estabilidade estocástica e estabilidade exponencial, formulados em termos de uma classe de funções de Lyapunov acopladas do tipo $V(x, r)=x^{T} P_{r} x$, com $P_{r}=P_{r}^{T}, P_{r}>0$, para $r \in \mathbb{S}$, que envolve a solução simultânea de um sistema de equações de Riccati acopladas (Boukas e Liu, 2001; Boukas e Yang, 1999a; Boukas e Yang, 1999b; Costa e Boukas, 1998; Feng et al., 1992). Tendo em vista a estabilização do SFSM, apresentamse as seguintes definições de estabilidade e estabilização estocástica, seguindo a mesma linha das definições para os SLSM.

Notação $3 \lambda_{\min }[\cdot]$ e $\lambda_{\max }[\cdot]$ denotam os autovalores mínimo e máximo, respectivamente, $\operatorname{tr}(\cdot)$ indica o traço de uma matriz, $\mathbf{I}$ e $\mathbf{0}$ denotam matrizes identidade $e$ zero de dimensões apropriadas, respectivamente, diag $\{\cdot\}$ denota uma matriz diagonal de dimensões apropriadas, $\sum_{\ell=1 ; \ell \neq i}^{N}$ denota, por exemplo para $N=3, \sum_{\ell=1 ; \ell \neq i}^{3} a_{i \ell} \Leftrightarrow$ $a_{12}+a_{13}+a_{21}+a_{23}+a_{31}+a_{32}, E[\cdot]$ denota o valor esperado de uma variável, $\|\cdot\|$ denota a norma Euclidiana para vetores ou a norma de uma matriz.

Definição 3 O estado de equilíbrio $x=0$ do SFSM (2.32) é dito ser 
1. assintoticamente estável na média-quadrática, se

$$
\lim _{t \rightarrow 0} E\left[\|x\|^{2}\right]=0
$$

2. exponencialmente estável na média-quadrática, se existem constantes positivas $c_{1}$ $e c_{2}$, tal que

$$
E\left[\|x\|^{2}\right] \leq c_{1}\left\|x_{0}\right\|^{2} e^{-c_{2} t}
$$

3. estocasticamente estável, se

$$
E\left[\int_{0}^{\infty}\|x\|^{2} d t\right]<\infty
$$

para todas as condições iniciais $\left(x_{0}, r_{0}\right)$.

Seja $x\left(t, x_{0}, r_{0}, u\right)$ a solução do SFSM (2.32) com lei de controle fuzzy (2.38) sob condições iniciais $\left(x_{0}, r_{0}\right)$.

Definição $4 O$ estado de equilíbrio $x=0$ do SFSM (2.32) é dito ser estocasticamente estável se, para todas as condições iniciais $\left(x_{0}, r_{0}\right)$, existe uma lei de controle fuzzy (2.38) satisfazendo

$$
\lim _{T \rightarrow \infty} E\left[\int_{0}^{T} x\left(t, x_{0}, r_{0}, u\right)^{T} x\left(t, x_{0}, r_{0}, u\right) d t \mid x_{0}, r_{0}\right] \leq x_{0}^{T} M x_{0}
$$

para alguma matriz $M=M^{T}, M>0$ de dimensões apropriadas.

As Definições 3 e 4 são similares aquelas de estabilidade e estabilização estocástica de SLSM (Boukas e Liu, 2001). Pela Definição 4, a estabilização estocástica do SFSM baseia-se na existência de uma lei de controle fuzzy $u$ que assintoticamente leva o estado do sistema $x$ de qualquer condição inicial $\left(x_{0}, r_{0}\right)$ à origem no sentido da média quadrática, que implica a estabilidade assintótica do sistema fuzzy em malha-fechada ou realimentado.

Proposição $3 O$ estado de equilíbrio $x=0$ do SFSM (2.32) com $u \equiv 0$ é estocasticamente estável se existe um conjunto de matrizes $P_{i}=P_{i}^{T}, P_{i}>0$ de dimensões 
apropriadas satisfazendo

$$
\left(A_{i j}-\frac{1}{2} \pi_{i} \mathbf{I}\right)^{T} P_{i}+P_{i}\left(A_{i j}-\frac{1}{2} \pi_{i} \mathbf{I}\right)+\sum_{\ell=1 ; \ell \neq i}^{N} \pi_{i \ell} P_{\ell}<0
$$

$\forall i \in \mathbb{S}$ e $j=1,2, \ldots, R$.

Prova: Considere que o SFSM (2.32) com $u \equiv 0$ no instante $t$ esteja no modo $i$, isto é, $r=i, i \in \mathbb{S}$. Também considere uma função de Lyapunov candidata do tipo

$$
V(x, i)=x^{T} P_{i} x
$$

onde $P_{i}:=P_{r=i}, P_{i}=P_{i}^{T}, P_{i}>0, i \in \mathbb{S}$. O operador infinitesimal fraco ${ }^{1}$ de (3.6) é dado por

$$
\mathcal{A} V(x, i):=\lim _{\delta \rightarrow 0} \frac{1}{\delta}\{E[V(x(t+\delta), r(t+\delta)) \mid x, i]-V(x, r=i)\}
$$

Usando Mariton (1990) é possível obter

$$
\begin{aligned}
\mathcal{A} V(x, i) & =\dot{x}^{T} \frac{\partial}{\partial x} V(x, i)+\sum_{\ell=1}^{N} \pi_{i \ell} V(x, \ell) \\
& =\dot{x}^{T} P_{i} x+x^{T} P_{i} \dot{x}+x^{T}\left(\sum_{\ell=1}^{N} \pi_{i \ell} P_{i}\right) x .
\end{aligned}
$$

Substituindo (2.32) com $u \equiv 0$ em (3.8) e tendo em vista o fato de que $m_{i}(z)=1$ quando $r=i$, obtém-se

$$
\mathcal{A} V(x, i)=\left(\sum_{j=1}^{R} n_{i j}(x) A_{i j} x\right)^{T} P_{i} x+x^{T} P_{i}\left(\sum_{j=1}^{R} n_{i j}(x) A_{i j} x\right)+x^{T}\left(\sum_{\ell=1}^{N} \pi_{i \ell} P_{i}\right) x(3.9)
$$

Tendo em vista que $\sum_{j=1}^{R} n_{i j}(x)=1$ e $\pi_{i}=-\pi_{i i}=\sum_{j=1, j \neq i}^{N} \pi_{i j}$, equação (3.9) pode

\footnotetext{
${ }^{1} \mathrm{O}$ operador infinitesimal fraco $\mathcal{A}$ de uma função de um processo estocástico corresponde ao operador de derivadas.
} 
ser reescrita na seguinte forma

$$
\begin{aligned}
\mathcal{A} V(x, i) & =\sum_{j=1}^{R} n_{i j}(x)\left[x^{T}\left(A_{i j}^{T} P_{i}+P_{i} A_{i j}+\sum_{\ell=1}^{N} \pi_{i \ell} P_{i}\right) x\right] \\
& =\sum_{j=1}^{R} n_{i j}(x)\left\{x^{T}\left[\left(A_{i j}-\frac{1}{2} \pi_{i} \mathbf{I}\right)^{T} P_{i}+P_{i}\left(A_{i j}-\frac{1}{2} \pi_{i} \mathbf{I}\right)+\sum_{\ell=1 ; \ell \neq i}^{N} \pi_{i \ell} P_{i}\right] x\right\} .
\end{aligned}
$$

Então, dado que $n_{i j}(x) \geq 0$, se a desigualdade (3.5) é satisfeita, tem-se $\mathcal{A} V(x, i)<0$ para $x \neq 0$.

Agora, objetivando verificar a estabilidade exponencial e conseqüentemente a estabilidade estocástica do SFSM (2.32) com $u \equiv 0$, define-se

$$
\mathcal{L}\left(\bar{A}_{i j}, P_{i}\right):=\bar{A}_{i j}^{T} P_{i}+P_{i} \bar{A}_{i j}+\sum_{\ell=1}^{N} \pi_{i \ell} P_{\ell}
$$

$\operatorname{com} \bar{A}_{i j}=\sum_{j=1}^{R} n_{i j}(x) A_{i j}$. Usando (3.11), pode-se reescrever (3.10) como

$$
\mathcal{A} V(x, i)=x^{T} \mathcal{L}\left(\bar{A}_{i j}, P_{i}\right) x
$$

para todo $i \in \mathbb{S}$. Desta forma, $\forall x \neq 0$, tem-se

$$
\begin{aligned}
\frac{\mathcal{A} V(x, i)}{V(x, i)} & =\frac{x^{T} \mathcal{L}\left(\bar{A}_{i j}, P_{i}\right) x}{x^{T} P_{i} x} \\
& \leq-\rho
\end{aligned}
$$

onde $\rho$ é uma constante positiva definida por $\rho:=\min _{i \in \mathbb{S}}\left\{\lambda_{\min }\left[-\mathcal{L}\left(\bar{A}_{i j}, P_{i}\right)\right] / \lambda_{\max }\left[P_{i}\right]\right\}$. Então, pela fórmula de Dynkin (Kushner, 1967), tem-se

$$
E[V(x(t), r(t))]-V\left(x_{0}, r_{0}\right)=E\left[\int_{0}^{t} \mathcal{A} V(x(s), r(s)) d s\right] .
$$

Substituindo (3.13) em (3.14), obtém-se

$$
\begin{aligned}
E[V(x(t), r(t))]-V\left(x_{0}, r_{0}\right) & \leq E\left[\int_{0}^{t}-\rho V(x(s), r(s)) d s\right] \\
& =-\rho \int_{0}^{t} E[V(x(s), r(s)) d s] .
\end{aligned}
$$


Usando o lema de Gronwall-Bellman (Khalil, 1996) em (3.15), tem-se

$$
\begin{aligned}
E[V(x(t), r(t))] & \leq V\left(x_{0}, r_{0}\right) e^{-\rho t} \\
& =\left(x_{0}^{T} P_{r} x_{0}\right) e^{-\rho t}
\end{aligned}
$$

Agora, integrando ambos os lados da equação (3.16) e tomando o seu limite quando $T \rightarrow \infty$, obtém-se

$$
\begin{aligned}
\lim _{T \rightarrow \infty} E\left[\int_{0}^{T} x^{T} P_{r} x d t \mid x_{0}, r_{0}\right] & \leq \frac{1}{\rho} x_{0}^{T} P_{r} x_{0} \\
& \leq \frac{1}{\rho} \lambda_{\max }\left[P_{r}\right]\left\|x_{0}\right\|^{2}
\end{aligned}
$$

Considerando o fato de que em (3.17) $P_{r}=P_{r}^{T}, P_{r}>0, \forall r \in \mathbb{S}$, o resultado segue pela Definição 3.

O conceito de estabilidade formalizado em termos dos parâmetros de cada modo, é conhecido como conceito "determinístico" ou "desacoplado" (Costa, 2002). Neste contexto, a estabilidade do SFSM em cada modo (ou seja, autovalores com parte real negativa) não é uma condição necessária nem suficiente para a estabilidade estocástica. Pela Proposição 3 a estabilidade estocástica do SFSM é dada em termos dos subsistemas em cada modo de operação e das taxas de transição do processo de Markov $\left(A_{i j}, \Pi\right)$, $i \in \mathbb{S}, j=1,2, \ldots, R$, logo a estabilidade em cada modo de operação é sempre verificada se $\operatorname{Re}\left\{\lambda\left[A_{i j}-\frac{1}{2} \pi_{i} \mathbf{I}\right]\right\}<0, \pi_{i}>0$.

Observação 6 Considerando que os elementos $\pi_{i j}$ da matriz de taxas de transição $\Pi$ podem ser especificados de maneira flexivel de acordo com o processo de Markov, mesmo que as matrizes $A_{i j}$ tenham autovalores instáveis, a estabilidade em cada modo ainda pode ser preservada especificando $\pi_{i}>2 \operatorname{Re}\left\{\lambda_{\max _{j}}\left[A_{i j}\right]\right\}$.

Considera-se agora a estabilização do SFSM (2.32) com lei de controle fuzzy (2.38).

Proposição $4 O$ estado de equilíbrio $x=0$ do SFSM (2.32) com lei de controle fuzzy (2.38) é estocasticamente estável se existe um conjunto de matrizes $P_{i}=P_{i}^{T}, P_{i}>$ 
0 de dimensões apropriadas satisfazendo $\forall i \in \mathbb{S}$

$$
\begin{gathered}
\left(G_{i j}-\frac{1}{4} \pi_{i} \mathbf{I}\right)^{T} P_{i}+P_{i}\left(G_{i j}-\frac{1}{4} \pi_{i} \mathbf{I}\right)+\sum_{\ell=1 ; \ell \neq i}^{N} \pi_{i \ell} P_{\ell}<0 \\
j=1,2, \ldots, R \\
\left(H_{i j k}-\frac{1}{4} \pi_{i} \mathbf{I}\right)^{T} P_{i}+P_{i}\left(H_{i j k}-\frac{1}{4} \pi_{i} \mathbf{I}\right)+\sum_{\ell=1 ; \ell \neq i}^{N} \pi_{i \ell} P_{\ell}<0 \\
j<k ; j, k=1,2, \ldots, R .
\end{gathered}
$$

Prova: Seja $i$ o modo do sistema no instante $t$, que é $r=i, i \in \mathbb{S}$. No que segue, por simplicidade de notação $x$ denota a solução $x\left(t, x_{0}, r_{0}, u\right)$ do SFSM $(2.32)$ sob condições iniciais $\left(x_{0}, r_{0}\right)$ com lei de controle fuzzy $(2.38)$. Considere a função de Lyapunov candidata (3.6) e seu operador infinitesimal fraco (3.8). Então, substituindo (2.41) em (3.8) e novamente tendo em vista o fato de que $m_{i}(z)=1$ quando $r=i$, obtém-se

$$
\begin{aligned}
\mathcal{A} V(x, i)= & \left(\sum_{j=1}^{R} n_{i j}^{2}(x) G_{i j} x+2 \sum_{j<k}^{R} n_{i j}(x) n_{i k}(x) H_{i j k} x\right)^{T} P_{i} x \\
& +x^{T} P_{i}\left(\sum_{j=1}^{R} n_{i j}^{2}(x) G_{i j} x+2 \sum_{j<k}^{R} n_{i j}(x) n_{i k}(x) H_{i j k} x\right)+x^{T}\left(\sum_{\ell=1}^{N} \pi_{i \ell} P_{i}\right) x
\end{aligned}
$$

Tendo em vista que $\sum_{j=1}^{R} \sum_{k=1}^{R} n_{i j}(x) n_{i k}(x)=\sum_{j=1}^{R} n_{i j}^{2}(x)+2 \sum_{j<k}^{R} n_{i j}(x) n_{i k}(x)=1$ e $\pi_{i}=-\pi_{i i}=\sum_{j=1, j \neq i}^{N} \pi_{i j},(3.20)$ pode ser reescrita como

$$
\begin{aligned}
\mathcal{A} V(x, i)= & x^{T}\left[\sum_{j=1}^{R} n_{i j}^{2}(x)\left(G_{i j}^{T} P_{i}+P_{i} G_{i j}\right)+2 \sum_{j<k}^{R} n_{i j}(x) n_{i k}(x)\left(H_{i j k}^{T} P_{i}+P_{i} H_{i j k}\right)\right. \\
& \left.+\left(\sum_{j=1}^{R} n_{i j}^{2}(x)+2 \sum_{j<k}^{R} n_{i j}(x) n_{i k}(x)\right)\left(\sum_{\ell=1}^{N} \pi_{i \ell} P_{\ell}\right)\right] x \\
= & x^{T}\left\{\sum_{j=1}^{R} n_{i j}^{2}(x)\left[\left(G_{i j}-\frac{1}{4} \pi_{i} \mathbf{I}\right)^{T} P_{i}+P_{i}\left(G_{i j}-\frac{1}{4} \pi_{i} \mathbf{I}\right)\right]\right. \\
& +2 \sum_{j<k}^{R} n_{i j}(x) n_{i k}(x)\left[\left(H_{i j k}-\frac{1}{4} \pi_{i} \mathbf{I}\right)^{T} P_{i}+P_{i}\left(H_{i j k}-\frac{1}{4} \pi_{i} \mathbf{I}\right)\right] \\
& \left.+\sum_{\ell=1 ; \ell \neq i}^{N} \pi_{i \ell} P_{\ell}\right\} x .
\end{aligned}
$$


Então, dado que $n_{i j}(x) \geq 0$, se as desigualdades (3.18) e (3.19) são satisfeitas, obtém-se $\mathcal{A} V(x, i)<0$ para $x \neq 0$. Agora, define-se

$$
\mathcal{L}\left(\bar{G}_{i j}, \bar{H}_{i j k}, P_{i}\right):=\left(\bar{G}_{i j}^{T} P_{i}+P_{i} \bar{G}_{i j}\right)+2\left(\bar{H}_{i j k}^{T} P_{i}+P_{i} \bar{H}_{i j k}\right)+\sum_{\ell=1}^{N} \pi_{i \ell} P_{\ell}
$$

onde $\bar{G}_{i j}=\sum_{j=1}^{R} n_{i j}^{2}(x) G_{i j}$ e $\bar{H}_{i j k}=\sum_{j<k}^{R} n_{i j}(x) n_{i k}(x) H_{i j k}$. Procedendo de forma similar à prova da Proposição 3, substitui-se (3.22) em (3.21) para obter

$$
\mathcal{A} V(x, i)=x^{T} \mathcal{L}\left(\bar{G}_{i j}, \bar{H}_{i j k}, P_{i}\right) x .
$$

Desta forma, tem-se para todo $x \neq 0$ e $i \in \mathbb{S}$

$$
\begin{aligned}
\frac{\mathcal{A} V(x, i)}{V(x, i)} & =\frac{x^{T} \mathcal{L}\left(\bar{G}_{i j}, \bar{H}_{i j k}, P_{i}\right) x}{x^{T} P_{i} x} \\
& \leq-\gamma
\end{aligned}
$$

onde $\gamma$ é uma constante positiva definida por $\gamma:=\min _{i \in \mathbb{S}}\left\{\lambda_{\min }\left[-\mathcal{L}\left(\bar{G}_{i j}, \bar{H}_{i j k}, P_{i}\right)\right] / \lambda_{\max }\left[P_{i}\right]\right\}$. Então, substituindo (3.24) em (3.14), obtém-se

$$
\begin{aligned}
E[V(x(t), r(t))]-V\left(x_{0}, r_{0}\right) & =E\left[\int_{0}^{t} \mathcal{A} V(x(s), r(s)) d s\right] \\
& \leq-\gamma \int_{0}^{t} E[V(x(s), r(s)) d s]
\end{aligned}
$$

Usando novamente o lema de Gronwall-Bellman, chega-se à seguinte desigualdade

$$
\begin{aligned}
E[V(x(t), r(t))] & \leq V\left(x_{0}, r_{0}\right) e^{-\gamma t} \\
& =\left(x_{0}^{T} P_{r} x_{0}\right) e^{-\gamma t}
\end{aligned}
$$

Integrando ambos os lados de (3.25) e tomando o limite quando $T \rightarrow \infty$, resulta

$$
\begin{aligned}
\lim _{T \rightarrow \infty} E\left[\int_{0}^{T} x^{T} P_{r} x d t \mid x_{0}, r_{0}\right] & \leq \frac{1}{\gamma} x_{0}^{T} P_{r} x_{0} \\
& \leq \frac{1}{\gamma} \lambda_{\max }\left[P_{r}\right] x_{0}^{T} x_{0}
\end{aligned}
$$

Novamente, considerando o fato que em (3.26) $P_{r}=P_{r}^{T}, P_{r}>0, \forall r \in \mathbb{S}$, o resultado segue pela Definição 4 . 


\subsection{Estabilização Fuzzy Estocástica}

Nesta seção, apresentam-se projetos de controladores fuzzy usando os resultados de estabilidade apresentados na Seção 3.1, que são formulados no contexto de análise convexa usando LMI's. Dado que $G_{i j}=A_{i j}-B_{i j} K_{i j}$ e $H_{i j k}=\frac{1}{2}\left(A_{i j}-B_{i j} K_{i k}+A_{i k}-B_{i k} K_{i j}\right)$, as desigualdades (3.18) e (3.19), podem ser escritas na seguinte forma

$$
\begin{array}{r}
A_{i j}^{T} P_{i}+P_{i} A_{i j}-K_{i j}^{T} B_{i j}^{T} P_{i}-P_{i} B_{i j} K_{i j}-\frac{1}{2} \pi_{i} \mathbf{I}+\sum_{\substack{\ell=1 ; \ell \neq i \\
j=1,2, \ldots, R}}^{N} \pi_{i \ell} P_{\ell}<0 \\
j=1,2,1
\end{array}
$$

$\mathrm{e}$

$$
\begin{array}{r}
A_{i j}^{T} P_{i}+P_{i} A_{i j}-K_{i k}^{T} B_{i j}^{T} P_{i}-P_{i} B_{i j} K_{i k} \\
+A_{i k}^{T} P_{i}+P_{i} A_{i k}-K_{i j}^{T} B_{i k}^{T} P_{i}-P_{i} B_{i k} K_{i j}-\frac{1}{2} \pi_{i} \mathbf{I}+\sum_{\substack{\ell=1 ; \ell \neq i \\
j<k}}^{N} \pi_{i \ell} P_{\ell}<0 ; \\
j, k=1,2, \ldots, R
\end{array}
$$

Note que as desigualdades (3.27) e (3.28) não são LMI's, pois aparecem produtos entre as variáveis $K_{i j}$ e $P_{i}$. No entanto, pré e pós-multiplicando as desigualdades (3.27) e (3.28) por $P_{i}^{-1}$ e definindo as seguintes variáveis

$$
\begin{gathered}
X_{i}:=P_{i}^{-1} \\
Y_{i j}:=K_{i j} X_{i}
\end{gathered}
$$

pode-se obter

$$
\begin{array}{r}
X_{i} A_{i j}^{T}+A_{i j} X_{i}-Y_{i j}^{T} B_{i j}^{T}-B_{i j} Y_{i j}-\frac{1}{2} \pi_{i} X_{i}+\sum_{\ell=1 ; \ell \neq i}^{N} \pi_{i \ell}\left(X_{i} X_{\ell}^{-1} X_{i}\right)<0 \\
j=1,2, \ldots, R
\end{array}
$$


e

$$
\begin{array}{r}
X_{i} A_{i j}^{T}+A_{i j} X_{i}-Y_{i k}^{T} B_{i j}^{T}-B_{i j} Y_{i k} \\
+X_{i} A_{i k}^{T}+A_{i k} X_{i}-Y_{i j}^{T} B_{i k}^{T}-B_{i k} Y_{i j}-\frac{1}{2} \pi_{i} X_{i}+\sum_{\substack{\ell=1 ; \ell \neq i \\
j<k ; j, k=1,2, \ldots, R .}}^{N} \pi_{i \ell}\left(X_{i} X_{\ell}^{-1} X_{i}\right)<0 \\
j<k, j=1, k
\end{array}
$$

que são LMI's nas variáveis $X_{i}$ e $Y_{i j}$. Agora, definindo como variáveis

$$
\begin{aligned}
& T_{i j}:=X_{i} A_{i j}^{T}+A_{i j} X_{i}-Y_{i j}^{T} B_{i j}^{T}-B_{i j} Y_{i j}-\frac{1}{2} \pi_{i} X_{i} \\
& \begin{aligned}
U_{i j k}:=\quad & X_{i} A_{i j}^{T}+A_{i j} X_{i}-Y_{i k}^{T} B_{i j}^{T}-B_{i j} Y_{i k} \\
& +X_{i} A_{i k}^{T}+A_{i k} X_{i}-Y_{i j}^{T} B_{i k}^{T}-B_{i k} Y_{i j}-\frac{1}{2} \pi_{i} X_{i}
\end{aligned} \\
& Z_{i}:=\left[\begin{array}{llllll}
\pi_{i 1}^{1 / 2} X_{i} & \ldots & \pi_{i i-1}^{1 / 2} X_{i} & \pi_{i i+1}^{1 / 2} X_{i} & \ldots & \pi_{i N}^{1 / 2} X_{i}
\end{array}\right] \\
& W_{i}:=\operatorname{diag}\left\{\begin{array}{llllll}
X_{1} & \ldots & X_{i-1} & X_{i+1} & \ldots & X_{N}
\end{array}\right\}
\end{aligned}
$$

e usando os complementos de Schur (Lema 1) em (3.31) e (3.32), chega-se às seguintes desigualdades matriciais

$$
\left[\begin{array}{ll}
T_{i j} & Z_{i} \\
Z_{i}^{T} & -W_{i}
\end{array}\right]<0 ; j=1,2, \ldots, R
$$

e

$$
\left[\begin{array}{ll}
U_{i j k} & Z_{i} \\
Z_{i}^{T} & -W_{i}
\end{array}\right]<0 ; j<k ; j, k=1,2, \ldots, R .
$$

Agora, usando (3.37) e (3.38), apresenta-se a seguinte proposição para a estabilização estocástica do SFSM.

Proposição 5 O estado de equilíbrio $x=0$ do SFSM (2.32) é estocasticamente estabilizável com lei de controle fuzzy (2.38) se existem um conjunto de matrizes $X_{i}=$ $X_{i}^{T}, X_{i}>0$ e um conjunto de matrizes $Y_{i j}$ de dimensões apropriadas satisfazendo as LMI's (3.37) e (3.38) $\forall i \in \mathbb{S}$, com $K_{i j}=Y_{i j} X_{i}^{-1}, j=1,2, \ldots, R$ os vetores de ganhos de realimentação de estado de dimensões apropriadas.

Prova: Segue diretamente da Proposição 4. 


\subsection{1 Índices de desempenho no projeto de controle}

Assim como a estabilidade, os índices de desempenho, tais como taxa de decaimento da resposta do sistema e restrição na entrada de controle têm um papel fundamental no projeto de controladores fuzzy. A velocidade de resposta de um sistema controlado, por exemplo, está relacionada com a taxa de decaimento, que é o maior expoente de Lyapunov. Em adição, existem aplicações práticas, nas quais o sinal de controle tem que ser limitado para garantir as condições de operação. No que segue, formula-se o projeto de controle fuzzy estabilizante usando os índices de desempenho mencionados.

\section{Taxa de decaimento}

Seja $\alpha_{i}:=\alpha_{r=i}, i \in \mathbb{S}$ uma constante positiva. Para incluir a taxa de decaimento no projeto de controle estabilizante é necessário que a seguinte condição seja satisfeita

$$
\mathcal{A} V(x, i) \leq-2 \alpha_{i} V(x, i)
$$

para cada modo $i \in \mathbb{S}$. No sentido determinístico, é possível verificar que a ação da restrição de taxa de decaimento (3.39) no projeto de controle fuzzy estabilizante produz um deslocamento para a esquerda nos autovalores das matrizes das representações lineares locais do SFSM, de forma que o tempo de resposta do sistema seja alterado, implicando maior velocidade de resposta.

Proposição 6 Considere que a taxa de decaimento $\alpha_{i}>0, i \in \mathbb{S}$ seja conhecida. A condição (3.39) é imposta para todas as trajetórias do SFSM (2.32) com lei de controle fuzzy (2.38), se existem um conjunto de matrizes $X_{i}=X_{i}^{T}, X_{i}>0$ e um conjunto de matrizes $Y_{i j}$ de dimensões apropriadas satisfazendo as seguintes LMI's $\forall i \in \mathbb{S}$

$$
\begin{aligned}
& {\left[\begin{array}{cc}
T_{i j} & Z_{i} \\
Z_{i}^{T} & -W_{i}
\end{array}\right]<-2 \alpha_{i}\left[\begin{array}{cc}
X_{i} & 0 \\
0 & 0
\end{array}\right] ; j=1,2, \ldots, R} \\
& {\left[\begin{array}{cc}
U_{i j k} & Z_{i} \\
Z_{i}^{T} & -W_{i}
\end{array}\right]<-2 \alpha_{i}\left[\begin{array}{cc}
X_{i} & 0 \\
0 & 0
\end{array}\right] ; j<k ; j, k=1,2, \ldots, R .}
\end{aligned}
$$

Prova: Considere novamente a função de Lyapunov candidata (3.6) e seu operador infinitesimal fraco (3.8). Substituindo (2.41) em (3.8) (veja Proposição 4) e usando 
(3.39), obtém-se

$$
\begin{array}{r}
x^{T}\left[\sum_{j=1}^{R} n_{i j}^{2}(x)\left(G_{i j}^{T} P_{i}+P_{i} G_{i j}\right)+2 \sum_{j<k}^{R} n_{i j}(x) n_{i k}(x)\left(H_{i j k}^{T} P_{i}+P_{i} H_{i j k}\right)+\sum_{\ell=1}^{N} \pi_{i \ell} P_{\ell}\right] x \\
\leq-2 \alpha_{i}\left(x^{T} P_{i} x\right) .
\end{array}
$$

Agora, usando os complementos de Schur nas LMI's (3.40) e (3.41) e substituindo (3.29), (3.30) e (3.33) - (3.36), chega-se às seguintes desigualdades

$$
G_{i j}^{T} P_{i}+P_{i} G_{i j}+\sum_{\ell=1}^{N} \pi_{i \ell} P_{\ell}<-2 \alpha_{i} P_{i} ; j=1,2, \ldots, R
$$

e

$$
H_{i j k}^{T} P_{i}+P_{i} H_{i j k}+\sum_{\ell=1}^{N} \pi_{i \ell} P_{\ell}<-2 \alpha_{i} P_{i} ; j<k ; j, k=1,2, \ldots, R .
$$

Multiplicando (3.43) por $n_{i j}^{2}(x)$ e (3.44) por $2 n_{i j}(x) n_{i k}(x)$, tem-se

$$
\sum_{j=1}^{R} n_{i j}^{2}(x)\left(G_{i j}^{T} P_{i}+P_{i} G_{i j}\right)+\sum_{j=1}^{R} n_{i j}^{2}(x)\left(\sum_{\ell=1}^{N} \pi_{i \ell} P_{\ell}\right)<-\sum_{j=1}^{R} n_{i j}^{2}(x)\left(2 \alpha_{i} P_{i}\right)
$$

$\mathrm{e}$

$$
\begin{array}{r}
2 \sum_{j<k}^{R} n_{i j}(x) n_{i k}(x)\left(H_{i j k}^{T} P_{i}+P_{i} H_{i j k}\right)+2 \sum_{j<k}^{R} n_{i j}(x) n_{i k}(x)\left(\sum_{\ell=1}^{N} \pi_{i \ell} P_{\ell}\right) \\
<-2 \sum_{j<k}^{R} n_{i j}(x) n_{i k}(x)\left(2 \alpha_{i} P_{i}\right) .
\end{array}
$$

Agora, adicionando (3.45) e (3.46) e usando o fato de que

$$
\sum_{j=1}^{R} \sum_{k=1}^{R} n_{i j}(x) n_{i k}(x)=\sum_{j=1}^{R} n_{i j}^{2}(x)+2 \sum_{j<k}^{R} n_{i j}(x) n_{i k}(x)=1
$$

chega-se a (3.42), o que completa a prova. 


\section{Restrição na entrada de controle}

Seja $\eta_{i}:=\eta_{r=i}, i \in \mathbb{S}$ uma constante positiva. No que segue, formula-se o projeto de controle fuzzy estabilizante adicionando a seguinte restrição no sinal de controle $\forall i \in \mathbb{S}$

$$
E\left[u^{T} u \mid x, r=i\right] \leq \eta_{i}^{2} .
$$

Proposição 7 Considere que a condição inicial $x_{0}$ seja conhecida. A restrição (3.47) é imposta para todas as trajetórias do SFSM (2.32) com lei de controle fuzzy (2.38), se as seguintes LMI's são asseguradas $\forall i \in \mathbb{S}$

$$
\begin{aligned}
& {\left[\begin{array}{cc}
1 & x_{0}^{T} \\
x_{0} & X_{i}
\end{array}\right] \geq 0 ;} \\
& {\left[\begin{array}{cc}
X_{i} & Y_{i j}^{T} \\
Y_{i j} & \eta_{i} \mathbf{I}
\end{array}\right] \geq 0 ; j=1,2, \ldots, R .}
\end{aligned}
$$

Prova: Considere que $V(x, i)$ em (3.6) é uma função de Lyapunov ao longo da trajetória do SFSM (2.41) em cada modo $i$. Substituindo (2.38) em (3.47), tem-se

$$
E\left[\left(\sum_{i=1}^{N} \sum_{j=1}^{R} m_{i}(z) n_{i j}(x) K_{i j} x\right)^{T}\left(\sum_{i=1}^{N} \sum_{j=1}^{R} m_{i}(z) n_{i j}(x) K_{i j} x\right) \mid x, r=i\right] \leq \eta_{i}^{2}
$$

Tendo em vista o fato de que $m_{i}(z) m_{k}(z)=0, i \neq k, i, k \in \mathbb{S}$ em (3.50) quando $r=i$, obtém-se

$$
E\left[\sum_{i=1}^{N} m_{i}^{2}(z)\left(\sum_{j=1}^{R} \sum_{k=1}^{R} n_{i j}(x) n_{i k}(x) x^{T} K_{i j}^{T} K_{i k} x\right) \mid x, r=i\right] \leq \eta_{i}^{2} .
$$

Visto que $m_{i}(z)=1$, quando $r=i$ e $m_{i}(z)=0$, caso contrário, (3.51) pode ainda ser escrita como

$$
\sum_{j=1}^{R} \sum_{k=1}^{R} n_{i j}(x) n_{i k}(x) x^{T}\left(\frac{1}{\eta_{i}^{2}} K_{i j}^{T} K_{i k}\right) x \leq 1
$$

Usando os complementos de Schur nas LMI's (3.48) e (3.49), chega-se às seguintes desigualdades

$$
x_{0}^{T} P_{i} x_{0} \leq 1
$$


$\mathrm{e}$

$$
\frac{1}{\eta_{i}^{2}} K_{i j}^{T} K_{i j}-P_{i} \leq 0 ; j=1,2, \ldots, R
$$

Agora, usa-se (3.54) para obter (3.52). Multiplicando ambos os lados de (3.54) por $n_{i j}(x)$ e tendo em vista o fato de que $\sum_{j=1}^{R} n_{i j}(x)=1$, obtém-se

$$
\sum_{j=1}^{R} n_{i j}(x) x^{T}\left(\frac{1}{\eta_{i}^{2}} K_{i j}^{T} K_{i j}-P_{i}\right) x \leq 0 .
$$

Pode ser mostrado que (Tanaka e Wang, 2001)

$$
\sum_{j=1}^{R} \sum_{k=1}^{R} n_{i j}(x) n_{i k}(x) x^{T}\left(\frac{1}{\eta_{i}^{2}} K_{i j}^{T} K_{i k}-P_{i}\right) x \leq \sum_{j=1}^{R} n_{i j}(x) x^{T}\left(\frac{1}{\eta_{i}^{2}} K_{i j}^{T} K_{i j}-P_{i}\right) x .
$$

Deste modo, usando (3.55) em (3.56), obtém-se

$$
\sum_{j=1}^{R} \sum_{k=1}^{R} n_{i j}(x) n_{i k}(x) x^{T}\left(\frac{1}{\eta_{i}^{2}} K_{i j}^{T} K_{i k}-P_{i}\right) x \leq 0
$$

que é o mesmo que

$$
\sum_{j=1}^{R} \sum_{k=1}^{R} n_{i j}(x) n_{i k}(x) x^{T}\left(\frac{1}{\eta_{i}^{2}} K_{i j}^{T} K_{i k}\right) x \leq x^{T} P_{i} x
$$

Finalmente, pelo fato de que $V(x, i) \leq x_{0}^{T} P_{i} x_{0}$ e usando (3.53), obtém-se (3.52) de (3.58) e o resultado segue.

O problema de projeto de controle fuzzy estabilizante com taxa de decaimento e restrição na entrada de controle pode ser formulado como a seguir.

Problema 2 Dados $\alpha_{i}>0$ e $\eta_{i}>0$, encontrar um conjunto de matrizes $X_{i}=$ $X_{i}^{T}, X_{i}>0$ e um conjunto de matrizes $Y_{i j}$ de dimensões apropriadas satisfazendo as LMI's (3.40), (3.41), (3.48) e (3.49) $\forall i \in \mathbb{S}$.

Se o resultado do Problema 2 for factível, os vetores de ganhos de realimentação de estado podem ser obtidos como $K_{i j}=Y_{i j} X_{i}^{-1}, j=1,2, \ldots, R$. 
Resultados preliminares com a utilização desta abordagem de estabilização fuzzy aplicada a um SEP sujeito a variações abruptas e estocásticas no carregamento modeladas como saltos Markovianos podem ser encontrados em Arrifano e Oliveira (n.d.b) e Arrifano et al. (2004).

\subsection{Controle Fuzzy de Custo Garantido}

O controle de custo garantido quadrático tem sido bastante considerado para a estabilização estocástica de SLSM. Com o uso desta técnica é possível obter um desempenho desejado para o sistema controlado mesmo considerando incertezas nos parâmetros do SLSM. Alguns exemplos de trabalhos envolvendo esta técnica podem ser encontrados em Boukas e Liu (2001), Boukas et al. (1999) e Costa e Boukas (1998). Nesta seção, formula-se um projeto de controle fuzzy estabilizante em termos do problema de custo garantido quadrático usando LMI's, seguindo o resultado para SLSM apresentado em Costa e Boukas (1998), bem como resultados de estabilidade estocástica apresentados na Seção 3.1.

Definição 5 O estado de equilíbrio $x=0$ do SFSM (2.32) é dito ser estocasticamente estável, se existe uma lei de controle fuzzy (2.38), tal que, um limitante sobre o indice de desempenho quadrático

$$
J\left(x_{0}, r_{0}, u\right)=E\left[\int_{0}^{\infty}\left(x^{T} Q_{r} x+u^{T} R_{r} u\right) d t\right]
$$

ao longo das trajetórias do sistema seja minimizado $\forall\left(x_{0}, r_{0}\right)$, com $Q_{r}=Q_{r}^{T}, Q_{r}>0$ e $R_{r}=R_{r}^{T}, R_{r}>0$ conjuntos de matrizes de ponderação de dimensões apropriadas escolhidas para produzir um desempenho desejado no sistema.

Definição 6 Se existe uma lei de controle fuzzy (2.38) e uma constante positiva definida $\delta$, tal que $J\left(x_{0}, r_{0}, u\right)<\delta$ ao longo das trajetórias do SFSM (2.41), então $\delta$ é um custo garantido e (2.38) é uma lei de controle de custo garantido.

Proposição 8 Se existe um conjunto de matrizes $P_{i}=P_{i}^{T}, P_{i}>0$ de dimensões 
apropriadas satisfazendo

$$
\begin{gathered}
\left(G_{i j}-\frac{1}{4} \pi_{i} \mathbf{I}\right)^{T} P_{i}+P_{i}\left(G_{i j}-\frac{1}{4} \pi_{i} \mathbf{I}\right)+\sum_{\ell=1 ; \ell \neq i}^{N} \pi_{i \ell} P_{\ell}+Q_{i}+\sum_{\nu=1}^{R} K_{i \nu}^{T} R_{i} K_{i \nu}<0 \\
j=1,2, \ldots, R \\
\left(H_{i j k}-\frac{1}{4} \pi_{i} \mathbf{I}\right)^{T} P_{i}+P_{i}\left(H_{i j k}-\frac{1}{4} \pi_{i} \mathbf{I}\right)+\sum_{\ell=1 ; \ell \neq i}^{N} \pi_{i \ell} P_{\ell}+Q_{i}+\sum_{\nu=1}^{R} K_{i \nu}^{T} R_{i} K_{i \nu}<0 ; \\
j<k ; j, k=1,2, \ldots, R
\end{gathered}
$$

$\forall i \in \mathbb{S}$, onde $Q_{r}=Q_{r}^{T}, Q_{r}>0$ e $R_{r}=R_{r}^{T}, R_{r}>0$ são conjuntos de matrizes de ponderação de dimensões apropriadas, então (2.38) é uma lei de controle fuzzy de custo garantido que estocasticamente estabiliza o SFSM (2.32) em torno do estado de equilíbrio $x=0$ e, se $E\left[\left\|x_{0}\right\|^{2}\right] \leq 1$, o funcional em (3.59) satisfaz a seguinte desigualdade

$$
J\left(x_{0}, r_{0}, u\right) \leq \delta
$$

onde $\delta=\sum_{i=1}^{N} \operatorname{tr}\left(P_{i}\right)$.

Prova: Inicialmente, prova-se que, se (3.60) e (3.61) são satisfeitas, o SFSM em malha-fechada é estocasticamente estável. Multiplicando (3.60) por $n_{i j}^{2}(x)$ e (3.61) por $2 n_{i j}(x) n_{i k}(x)$, obtém-se

$$
\begin{aligned}
& \sum_{j=1}^{R} n_{i j}^{2}(x)\left[\left(G_{i j}-\right.\right.\left.\left.\frac{1}{4} \pi_{i} \mathbf{I}\right)^{T} P_{i}+P_{i}\left(G_{i j}-\frac{1}{4} \pi_{i} \mathbf{I}\right)\right]+\sum_{j=1}^{R} n_{i j}^{2}(x) \sum_{\ell=1 ; \ell \neq i}^{N} \pi_{i \ell} P_{\ell} \\
&<-\sum_{j=1}^{R} n_{i j}^{2}(x)\left(Q_{i}+\sum_{\nu=1}^{R} K_{i \nu}^{T} R_{i} K_{i \nu}\right)
\end{aligned}
$$

e

$$
\begin{gathered}
\sum_{j<k}^{R} n_{i j}(x) n_{i k}(x)\left[\left(H_{i j k}-\frac{1}{4} \pi_{i} \mathbf{I}\right)^{T} P_{i}+P_{i}\left(H_{i j k}-\frac{1}{4} \pi_{i} \mathbf{I}\right)\right] \\
+\sum_{j<k}^{R} n_{i j}(x) n_{i k}(x) \sum_{\ell=1 ; \ell \neq i}^{N} \pi_{i \ell} P_{\ell}<-\sum_{j<k}^{R} n_{i j}(x) n_{i k}(x)\left(Q_{i}+\sum_{\nu=1}^{R} K_{i \nu}^{T} R_{i} K_{i \nu}\right) .
\end{gathered}
$$

Adicionando (3.63) a (3.64) e, novamente, tendo em vista que $\sum_{j=1}^{R} \sum_{k=1}^{R} n_{i j}(x) n_{i k}(x)=$ 


$$
\begin{aligned}
& \sum_{j=1}^{R} n_{i j}^{2}(x)+2 \sum_{j<k}^{R} n_{i j}(x) n_{i k}(x)=1 \text {, resulta } \\
& \quad \sum_{j=1}^{R} n_{i j}^{2}(x)\left[\left(G_{i j}-\frac{1}{4} \pi_{i} \mathbf{I}\right)^{T} P_{i}+P_{i}\left(G_{i j}-\frac{1}{4} \pi_{i} \mathbf{I}\right)\right] \\
& \quad+2 \sum_{j<k}^{R} n_{i j}(x) n_{i k}(x)\left[\left(H_{i j k}-\frac{1}{4} \pi_{i} \mathbf{I}\right)^{T} P_{i}+P_{i}\left(H_{i j k}-\frac{1}{4} \pi_{i} \mathbf{I}\right)\right]+\sum_{\ell=1 ; \ell \neq i}^{N} \pi_{i \ell} P_{\ell} \\
& \quad<-\left(Q_{i}+\sum_{\nu=1}^{R} K_{i \nu}^{T} R_{i} K_{i \nu}\right) .
\end{aligned}
$$

Então, pré-multiplicando (3.65) por $x^{T}$ e pós-multiplicando por $x$, pela Proposição 4 , pode-se verificar que

$$
\mathcal{A} V(x, i)<-x^{T}\left(Q_{i}+\sum_{\nu=1}^{R} K_{i \nu}^{T} R_{i} K_{i \nu}\right) x
$$

Logo, dado que $Q_{i}>0$ e $R_{i}>0$, tem-se $Q_{i}+\sum_{\nu=1}^{R} K_{i \nu}^{T} R_{i} K_{i \nu}>0$ e pela Proposição 4 , verifica-se que o SFSM em malha-fechada é estocasticamente estável.

Agora, considere que o SFSM em malha-fechada (2.41) no instante $t$ esteja no modo $i$, isto é, $r=i, i \in \mathbb{S}$, então, o funcional $J\left(x_{0}, r_{0}, u\right)$ formulado em termos da lei de controle fuzzy (2.38) é obtido substituindo (2.38) em (3.59), que resulta

$$
J\left(x_{0}, r_{0}, u\right)=E\left[\int_{0}^{\infty}\left[x^{T} Q_{i} x+\left(\sum_{\nu=1}^{R} n_{i \nu}(x) K_{i \nu} x\right)^{T} R_{i}\left(\sum_{\nu=1}^{R} n_{i \nu}(x) K_{i \nu} x\right)\right] d t\right]
$$

Pode ser mostrado que (Jadbabaie et al., 1998)

$$
\left(\sum_{\nu=1}^{R} n_{i \nu}(x) K_{i \nu}\right)^{T} R_{i}\left(\sum_{\nu=1}^{R} n_{i \nu}(x) K_{i \nu}\right)<\sum_{\nu=1}^{R} K_{i \nu}^{T} R_{i} K_{i \nu}
$$

Desta forma, usando (3.68) em (3.67), obtém-se

$$
J\left(x_{0}, r_{0}, u\right)<E\left[\int_{0}^{\infty} x^{T}\left[Q_{i}+\left(\sum_{\nu=1}^{R} K_{i \nu}^{T} R_{i} K_{i \nu}\right)\right] x d t\right] .
$$


Integrando ambos os lados de (3.66), obtém-se

$$
E\left[\int_{0}^{t} \mathcal{A} V(x(s), r(s)) d s\right]<-E\left[\int_{0}^{t} x(s)^{T}\left(Q_{r}+\sum_{\nu=1}^{R} K_{r \nu}^{T} R_{r} K_{r \nu}\right) x(s) d s\right]
$$

Novamente, pela fórmula de Dynkin, tem-se

$$
E[V(x(t), r(t))]-V\left(x_{0}, r_{0}\right)=E\left[\int_{0}^{t} \mathcal{A} V(x(s), r(s)) d s\right]
$$

Assim, substituindo (3.71) em (3.70), obtém-se

$$
E[V(x(t), r(t))]-V\left(x_{0}, r_{0}\right)<-E\left[\int_{0}^{t} x(s)^{T}\left(Q_{r}+\sum_{\nu=1}^{R} K_{r \nu}^{T} R_{r} K_{r \nu}\right) x(s) d s\right]
$$

Então, dado que $V\left(x_{0}, r_{0}=i\right)=x_{0}^{T} P_{i} x_{0}=\operatorname{tr}\left(P_{i} X_{i}(0)\right), P_{i}=P_{i}^{T}, P_{i}>0$ e $X_{i}(0):=$ $x_{0} x_{0}^{T}$, tomando o limite de (3.72) quando $t \rightarrow \infty$ e tendo em vista o funcional $J\left(x_{0}, r_{0}, u\right)$ em (3.69), tem-se

$$
\begin{aligned}
J\left(x_{0}, r_{0}, u\right) & <\sum_{i=1}^{N} \operatorname{tr}\left(P_{i} X_{i}(0)\right) \\
& \leq \sum_{i=1}^{N} \operatorname{tr}\left(P_{i}\right) \\
& =\delta
\end{aligned}
$$

para $x_{0}$, tal que, $E\left[\left\|x_{0}\right\|^{2}\right]=\sum_{i=1}^{N} \operatorname{tr}\left(X_{i}(0)\right) \leq 1$, o que completa esta prova.

No que segue, as desigualdades (3.60) e (3.61) são escritas como LMI's. Dados $G_{i j}=A_{i j}-B_{i j} K_{i j}$ e $H_{i j k}=\frac{1}{2}\left(A_{i j}-B_{i j} K_{i k}+A_{i k}-B_{i k} K_{i j}\right)$, as desigualdades (3.60) e (3.61) podem ser reescritas na seguinte forma

$$
\begin{array}{r}
A_{i j}^{T} P_{i}+P_{i} A_{i j}-K_{i j}^{T} B_{i j}^{T} P_{i}-P_{i} B_{i j} K_{i j}-\frac{1}{2} \pi_{i} \mathbf{I}+\sum_{\ell=1 ; \ell \neq i}^{N} \pi_{i \ell} P_{\ell} \\
+Q_{i}+\sum_{\nu=1}^{R} K_{i \nu}^{T} R_{i} K_{i \nu}<0 ; j=1,2, \ldots, R
\end{array}
$$


e

$$
\begin{array}{r}
A_{i j}^{T} P_{i}+P_{i} A_{i j}-K_{i k}^{T} B_{i j}^{T} P_{i}-P_{i} B_{i j} K_{i k} \\
+A_{i k}^{T} P_{i}+P_{i} A_{i k}-K_{i j}^{T} B_{i k}^{T} P_{i}-P_{i} B_{i k} K_{i j}-\frac{1}{2} \pi_{i} \mathbf{I}+\sum_{\ell=1 ; \ell \neq i}^{N} \pi_{i \ell} P_{\ell} \\
+Q_{i}+\sum_{\nu=1}^{R} K_{i \nu}^{T} R_{i} K_{i \nu}<0 ; j<k ; j, k=1,2, \ldots, R .
\end{array}
$$

Novamente, pode-se notar que as desigualdades (3.74) e (3.75) não são LMI's, pois aparecem produtos entre as variáveis $K_{i j}$ e $P_{i}$. No entanto, pré e pós-multiplicando estas desigualdades por $P_{i}^{-1}$ e substituindo (3.29) e (3.30), tem-se

$$
\begin{array}{r}
X_{i} A_{i j}^{T}+A_{i j} X_{i}-Y_{i j}^{T} B_{i j}^{T}-B_{i j} Y_{i j}-\frac{1}{2} \pi_{i} X_{i}+\sum_{\ell=1 ; \ell \neq i}^{N} \pi_{i \ell}\left(X_{i} X_{\ell}^{-1} X_{i}\right) \\
+X_{i} Q_{i} X_{i}+\sum_{\nu=1}^{R} Y_{i \nu}^{T} R_{i} Y_{i \nu}<0 ; j=1,2, \ldots, R
\end{array}
$$

e

$$
\begin{array}{r}
X_{i} A_{i j}^{T}+A_{i j} X_{i}-Y_{i k}^{T} B_{i j}^{T}-B_{i j} Y_{i k} \\
+X_{i} A_{i k}^{T}+A_{i k} X_{i}-Y_{i j}^{T} B_{i k}^{T}-B_{i k} Y_{i j}-\frac{1}{2} \pi_{i} X_{i}+\sum_{\ell=1 ; \ell \neq i}^{N} \pi_{i \ell}\left(X_{i} X_{\ell}^{-1} X_{i}\right) \\
+X_{i} Q_{i} X_{i}+\sum_{\nu=1}^{R} Y_{i \nu}^{T} R_{i} Y_{i \nu}<0 ; j<k ; j, k=1,2, \ldots, R .
\end{array}
$$

Agora, definem-se

$$
\bar{T}_{i j}:=\left[\begin{array}{ccccc}
T_{i j} & X_{i} Q_{i}^{1 / 2} & Y_{i 1}^{T} R_{i}^{1 / 2} & \ldots & Y_{i R}^{T} R_{i}^{1 / 2} \\
Q_{i}^{1 / 2} X_{i} & -\mathbf{I} & \mathbf{0} & \ldots & \mathbf{0} \\
R_{i}^{1 / 2} Y_{i 1} & \mathbf{0} & -\mathbf{I} & \ldots & \mathbf{0} \\
\vdots & \vdots & \vdots & \vdots & \vdots \\
R_{i}^{1 / 2} Y_{i R} & \mathbf{0} & \mathbf{0} & \ldots & -\mathbf{I}
\end{array}\right]
$$


e

$$
\bar{U}_{i j k}:=\left[\begin{array}{ccccc}
U_{i j k} & X_{i} Q_{i}^{1 / 2} & Y_{i 1}^{T} R_{i}^{1 / 2} & \cdots & Y_{i R}^{T} R_{i}^{1 / 2} \\
Q_{i}^{1 / 2} X_{i} & -\mathbf{I} & \mathbf{0} & \ldots & \mathbf{0} \\
R_{i}^{1 / 2} Y_{i 1} & \mathbf{0} & -\mathbf{I} & \ldots & \mathbf{0} \\
\vdots & \vdots & \vdots & \vdots & \vdots \\
R_{i}^{1 / 2} Y_{i R} & \mathbf{0} & \mathbf{0} & \ldots & -\mathbf{I}
\end{array}\right]
$$

para $T_{i j}, U_{i j k}$ como em (3.33) e (3.34), respectivamente. Então, usando (3.78), (3.79), (3.35) e (3.36) e os complementos de Schur, chega-se às seguintes desigualdades matriciais

$$
\left[\begin{array}{ll}
\bar{T}_{i j} & Z_{i} \\
Z_{i}^{T} & -W_{i}
\end{array}\right]<0 ; j=1,2, \ldots, R
$$

$\mathrm{e}$

$$
\left[\begin{array}{ll}
\bar{U}_{i j k} & Z_{i} \\
Z_{i}^{T} & -W_{i}
\end{array}\right]<0 ; j<k ; j, k=1,2, \ldots, R .
$$

Considerando a Proposição 8 e usando as LMI's (3.80) e (3.81), formula-se o seguinte problema de otimização para o projeto de controle de controle fuzzy de custo garantido.

Problema 3 Encontrar um conjunto de matrizes $X_{i}=X_{i}^{T}, X_{i}>0$ e um conjunto de matrizes $Y_{i j}$ de dimensões apropriadas minimizando $\delta=\sum_{\ell=1}^{N} \operatorname{tr}\left(S_{i}\right)$ sujeito a

$$
\text { (3.80), (3.81), } X_{i}>0, S_{i}>0 e\left[\begin{array}{ll}
S_{i} & \mathbf{I} \\
\mathbf{I} & X_{i}
\end{array}\right] \geq 0 \text {. }
$$

Se o Problema 3 for factível, pode-se obter os ganhos de realimentação de estado usando $K_{i j}=Y_{i j} X_{i}^{-1}$. Note que a solução do problema de minimização implica $S_{i}=X_{i}^{-1}=P_{i}$. 


\section{Capítulo 4}

\section{Estabilização Fuzzy Robusta}

\subsection{Formulação Preliminar do Problema}

No capítulo anterior foram apresentados resultados de estabilidade e estabilização para o SFSM. Em geral, as técnicas de projeto de controle fuzzy ignoram o erro de aproximação entre o sistema não-linear e o sistema fuzzy. Entretanto, na prática, o efeito do erro de aproximação pode deteriorar a estabilidade e o desempenho da lei de controle no sistema não-linear. Por este motivo, uma técnica de projeto de controle fuzzy que garanta estabilidade e desempenho robusto é a técnica mais indicada para sistemas não-lineares. Recentemente, algumas técnicas de estabilização que englobam o erro de aproximação entre o sistema não-linear e o sistema fuzzy no projeto de controle fuzzy apareceram (Machado et al., 2003; Taniguchi et al., 2001; Chen et al., 1999). Em Machado et al. (2003) e em Taniguchi et al. (2001) são apresentadas técnicas de projeto de controle fuzzy que consideram uma redução no erro de aproximação. Nestas técnicas, o efeito do aumento do número de regras de inferência ocasionado pela aproximação setorial tem sido tratado, explorando tanto a escolha das funções de pertinência normalizadas, bem como suas propriedades. Em Chen et al. (1999), em vez de uma técnica de projeto de controle fuzzy baseada em uma aproximação setorial, é apresentada uma técnica de projeto de controle fuzzy com desempenho $\mathcal{H}_{\infty}$ que estabiliza o sistema não-linear e ao mesmo tempo elimina os efeitos de um distúrbio externo desconhecido abaixo de um nível prescrito pelo projeto, tal que, o desempenho do controle possa ser garantido. Neste capítulo, formula-se um projeto de controle fuzzy com desempenho $\mathcal{H}_{\infty}$ seguindo Chen et al. (1999) no contexto de LMI's para estabilização robusta da classe de SFSM 
sujeita a um distúrbio externo desconhecido. Para facilitar a apresentação da técnica, algumas equações do Capítulo 2 são reapresentadas a seguir.

Notação $4\|\cdot\|_{2}$ denota a norma no espaço $\mathcal{L}_{2}[0, T]$ e $E\|\cdot\|_{2}$ denota a norma no espaço $\mathcal{L}_{2}((\Omega, \mathcal{F}, \operatorname{Pr}),[0, T])$.

Seja $w \in \mathbb{R}^{n}$ um distúrbio externo desconhecido, porém limitado, pertencente ao espaço $\mathcal{L}_{2}[0, \infty]$. Considere as seguintes representações na forma espaço de estado do SNLSM (2.29) - (2.30) e do SFSM (2.32) com o distúrbio $w$

$$
\dot{x}=f(x, r)+g(x, r) u+w
$$

e

$$
\dot{x}=\sum_{i=1}^{N} \sum_{j=1}^{R} m_{i}(z) n_{i j}(x)\left(A_{i j} x+B_{i j} u\right)+w
$$

respectivamente. Agora, suponha que a lei de controle fuzzy com realimentação de estado

$$
u=-\sum_{i=1}^{N} \sum_{j=1}^{R} m_{i}(z) n_{i j}(x) K_{i j} x
$$

seja utilizada para controlar o sistema (4.2). Então, substituindo (4.3) em (4.2) e seguindo um procedimento similar ao da obtenção do SFSM em malha-fechada (2.41), obtém-se

$$
\dot{x}=\sum_{i=1}^{N} m_{i}(z)\left[\sum_{j=1}^{R} n_{i j}^{2}(x) G_{i j}+2 \sum_{j<k}^{R} n_{i j}(x) n_{i k}(x) H_{i j k}\right] x+w
$$

onde $G_{i j}=A_{i j}-B_{i j} K_{i j}$ e $H_{i j k}=\frac{1}{2}\left(A_{i j}-B_{i j} K_{i k}+A_{i k}-B_{i k} K_{i j}\right)$. Assim sendo, o sistema (4.1) pode ser descrito na seguinte representação com incertezas

$$
\dot{x}=\sum_{i=1}^{N} m_{i}(z)\left[\sum_{j=1}^{R} n_{i j}^{2}(x) G_{i j}+2 \sum_{j<k}^{R} n_{i j}(x) n_{i k}(x) H_{i j k}\right] x+\Delta f+\Delta g+w(4
$$


onde $\Delta f$ e $\Delta g$ são os erros de aproximação definidos por

$$
\Delta f:=\sum_{i=1}^{N} m_{i}(z)\left[\sum_{j=1}^{R} n_{i j}(x)\left(f(x, r)-A_{i j} x\right)\right]
$$

e

$$
\Delta g:=-\sum_{i=1}^{N} m_{i}(z)\left[\sum_{j=1}^{R} \sum_{k=1}^{R} n_{i j}(x) n_{i k}(x)\left(g(x, r)-B_{i j}\right) K_{i k} x\right]
$$

Para cada modo $i, i \in \mathbb{S}$, suponha que existem matrizes $\Delta A_{i j}$ e $\Delta B_{i j}, j=1,2, \ldots, R$ , tais que os erros de aproximação (4.6) e (4.7) satisfazem

$$
\|\Delta f\| \leq\left\|\sum_{j=1}^{R} n_{i j}(x) \Delta A_{i j} x\right\|
$$

e

$$
\|\Delta g\| \leq\left\|\sum_{j=1}^{R} \sum_{k=1}^{R} n_{i j}(x) n_{i k}(x) \Delta B_{i j} K_{i k} x\right\|
$$

ao longo da trajetória de $x$. Note que as matrizes $\Delta A_{i j}$ e $\Delta B_{i j}$ dependem das funções $f(x, r)$ e $g(x, r)$ e das matrizes das representações lineares locais $A_{i j}$ e $B_{i j}$, respectivamente. Em (4.8) e (4.9, as matrizes $\Delta A_{i j}$ e $\Delta B_{i j}$ podem ser descritas por

$$
\Delta A_{i j}=\delta_{i j} A_{\mathbf{p} i}
$$

$\mathrm{e}$

$$
\Delta B_{i j}=\eta_{i j} B_{\mathbf{p} i}
$$

onde $\left\|\delta_{i j}\right\| \leq 1$ e $\left\|\eta_{i j}\right\| \leq 1, j=1,2, \ldots, R$ e $A_{\mathbf{p} i}$ e $B_{\mathbf{p} i}$ são matrizes limitantes para $\Delta A_{i j}$ e $\Delta B_{i j}$ que podem ser escolhidas de acordo com a não-linearidade a ser aproxi- 
mada (Boyd et al., 1994). Deste modo, tem-se

$$
\begin{aligned}
\Delta f^{T} \Delta f & =\left[\sum_{j=1}^{R} n_{i j}(x)\left(f(x, i)-A_{i j} x\right)\right]^{T}\left[\sum_{j=1}^{R} n_{i j}(x)\left(f(x, i)-A_{i j} x\right)\right] \\
& \leq\left(\sum_{j=1}^{R} n_{i j}(x) \Delta A_{i j} x\right)^{T}\left(\sum_{j=1}^{R} n_{i j}(x) \Delta A_{i j} x\right) \\
& =\left(\sum_{j=1}^{R} n_{i j}(x) \delta_{i j} A_{\mathbf{p} i} x\right)^{T}\left(\sum_{j=1}^{R} n_{i j}(x) \delta_{i j} A_{\mathbf{p} i} x\right) \\
& \leq x^{T}\left(A_{\mathbf{p} i}^{T} A_{\mathbf{p} i}\right) x
\end{aligned}
$$

e

$$
\begin{aligned}
\Delta g^{T} \Delta g= & {\left[\sum_{j=1}^{R} \sum_{k=1}^{R} n_{i j}(x) n_{i k}(x)\left(g(x, i)-B_{i j}\right) K_{i k} x\right]^{T} } \\
& {\left[\sum_{j=1}^{R} \sum_{k=1}^{R} n_{i j}(x) n_{i k}(x)\left(g(x, i)-B_{i j}\right) K_{i k} x\right] } \\
\leq & {\left[\sum_{j=1}^{R} \sum_{k=1}^{R} n_{i j}(x) n_{i k}(x) \Delta B_{i j} K_{i k} x\right]^{T}\left[\sum_{j=1}^{R} \sum_{k=1}^{R} n_{i j}(x) n_{i k}(x) \Delta B_{i j} K_{i k} x\right] } \\
= & {\left[\sum_{j=1}^{R} \sum_{k=1}^{R} n_{i j}(x) n_{i k}(x) \eta_{i j} B_{\mathbf{p} i} K_{i k} x\right]^{T}\left[\sum_{j=1}^{R} \sum_{k=1}^{R} n_{i j}(x) n_{i k}(x) \eta_{i j} B_{\mathbf{p} i} K_{i k} x\right] } \\
\leq & x^{T}\left(\sum_{k=1}^{R} n_{i k}(x) B_{\mathbf{p} i} K_{i k}\right)^{T}\left(\sum_{k=1}^{R} n_{i k}(x) B_{\mathbf{p} i} K_{i k}\right) x .
\end{aligned}
$$

Tendo em vista que

$$
\sum_{k=1}^{R}\left(B_{\mathbf{p} i} K_{i k}\right)^{T}\left(B_{\mathbf{p} i} K_{i k}\right)>\left(\sum_{k=1}^{R} n_{i k}(x) B_{\mathbf{p} i} K_{i k}\right)^{T}\left(\sum_{k=1}^{R} n_{i k}(x) B_{\mathbf{p} i} K_{i k}\right)
$$

a desigualdade (4.13) pode ser reescrita como

$$
\Delta g^{T} \Delta g<x^{T}\left[\sum_{k=1}^{R}\left(B_{\mathbf{p} i} K_{i k}\right)^{T}\left(B_{\mathbf{p} i} K_{i k}\right)\right] x
$$




\subsection{Controle Fuzzy de Desempenho $\mathcal{H}_{\infty}$ Robusto}

Um importante problema em sistemas de controle é eliminar o efeito de $w$ para garantir o desempenho do controlador. Neste contexto, a técnica de projeto de controle $\mathcal{H}_{\infty}$ é a técnica mais indicada para atenuação do efeito de $w$ no sistema em malha-fechada ou controlado, por isso será utilizada para garantir o desempenho robusto do controlador fuzzy.

Seja $x\left(t, x_{0}, r_{0}, u\right)$ a solução do SFSM (4.2) com lei de controle fuzzy (4.3) sob condições iniciais $\left(x_{0}, r_{0}\right)$.

Definição 7 O estado de equilíbrio $x=0$ do SFSM (4.2) com $w \equiv 0$ é dito ser estocasticamente estável, se para todas as condições iniciais $\left(x_{0}, r_{0}\right)$ e incertezas na modelagem admissiveis, existe uma lei de controle fuzzy (4.3) satisfazendo

$$
E\left[\int_{0}^{\infty} x\left(t, x_{0}, r_{0}, u\right)^{T} x\left(t, x_{0}, r_{0}, u\right) d t \mid x_{0}, r_{0}\right] \leq x_{0}^{T} M x_{0}
$$

para alguma matriz $M=M^{T}, M>0$ de dimensões apropriadas.

Esta definição é similar àquela de estabilização estocástica de SLSM com nãolinearidades desconhecidas (Boukas et al., 2003; Mahmoud e Shi, 2002). Por esta definição, a estabilidade estocástica do SFSM (4.2) com $w \equiv 0$ considera a existência da lei de controle fuzzy (4.3) que assintoticamente leva o estado do sistema $x\left(t, x_{0}, r_{0}, u\right)$ de uma dada condição inicial $\left(x_{0}, r_{0}\right)$ à origem no sentido da média quadrática, implicando a estabilidade assintótica do sistema em malha-fechada. O problema de controle tratado aqui consiste na obtenção dos ganhos de realimentação de estado $K_{i j}, i \in \mathbb{S}, j=$ $1,2, \ldots, R$ para o controlador fuzzy (4.3), que estocasticamente estabiliza o SFSM (4.2) e atenua o efeito do distúrbio $w$. A atenuação do efeito de um distúrbio externo é então formulada em termos da norma $\mathcal{H}_{\infty}$ do sistema.

Definição 8 Considere o SFSM (4.5). Seja $\gamma$ uma constante positiva, então o mapemanto de $w$ a $x$ é dito ter ganho $\mathcal{L}_{2}((\Omega, \mathcal{F}, \operatorname{Pr}),[0, T])$ menor ou igual a $\gamma$, se para todo estado inicial $x_{0}, x_{0}<\infty$, modo inicial $r_{0}$ e lei de controle fuzzy (4.3), a seguinte 
restrição

$$
E\left[\int_{0}^{T} x^{T} S_{r} x d t\right]-\gamma^{2} \int_{0}^{T} w^{T} w d t<V\left(x_{0}, r_{0}\right)-E[V(x(T), r(T))]
$$

é assegurada para todas as incertezas na modelagem admissiveis, onde $S_{r}=S_{r}^{T}, S_{r}>0$ é um conjunto de matrizes de ponderação de dimensões apropriadas escolhidas para produzir um desempenho desejado no sistema.

Seja $T_{\zeta w}$ a matriz de transferência do sistema da entrada $w$ à saída regulada $\zeta:=$ $S_{r}^{1 / 2} x$. Então, a norma $\mathcal{H}_{\infty}$ de $T_{\zeta w}$ é dada por

$$
\left\|T_{\zeta w}\right\|_{\infty}=\sup _{w \in \mathcal{L}_{2}[0, T]} \frac{E\|\zeta\|_{2}}{\|w\|_{2}}
$$

Desta forma, (4.16) implica $\left\|T_{\zeta w}\right\|_{\infty}<\gamma$ e o problema de atenuação de distúrbio $\gamma$ corresponde ao problema de controle $\mathcal{H}_{\infty}$ sub-ótimo.

No que segue, apresentam-se condições suficientes para a estabilização estocástica do SFSM (4.2) usando a função de Lyapunov acoplada (3.6) e seu operador infinitesimal fraco $(3.8)$

$$
V(x, i)=x^{T} P_{i} x
$$

e

$$
\mathcal{A} V(x, i)=\dot{x}^{T} P_{i} x+x^{T} P_{i} \dot{x}+x^{T}\left(\sum_{\ell=1}^{N} \pi_{i \ell} P_{i}\right) x
$$

respectivamente, repetidos aqui para fácil referência, com $P_{i}:=P_{r=i}, P_{i}=P_{i}^{T}, P_{i}>0$, $i \in \mathbb{S}$.

Proposição 9 O estado de equilíbrio $x=0$ do SFSM (4.2) com $w \equiv 0$ é estocasticamente estável com lei de controle fuzzy (4.3) se, para todas as condições iniciais $\left(x_{0}, r_{0}\right)$ e todas as incertezas na modelagem admissiveis, existe um conjunto de matrizes 
$P_{i}=P_{i}^{T}, P_{i}>0$ de dimensões apropriadas satisfazendo $\forall i \in \mathbb{S}$

$$
\begin{gathered}
\left(G_{i j}-\frac{1}{4} \pi_{i} \mathbf{I}\right)^{T} P_{i}+P_{i}\left(G_{i j}-\frac{1}{4} \pi_{i} \mathbf{I}\right)+\sum_{\ell=1 ; \ell \neq i}^{N} \pi_{i \ell} P_{\ell} \\
+A_{\mathbf{p} i}^{T} A_{\mathbf{p} i}+\sum_{\nu=1}^{R}\left(B_{\mathbf{p} i} K_{i \nu}\right)^{T}\left(B_{\mathbf{p} i} K_{i \nu}\right)+2 P_{i} P_{i}<0 ; j=1,2, \ldots, R \\
\left(H_{i j k}-\frac{1}{4} \pi_{i} \mathbf{I}\right)^{T} P_{i}+P_{i}\left(H_{i j k}-\frac{1}{4} \pi_{i} \mathbf{I}\right)+\sum_{\ell=1 ; \ell \neq i}^{N} \pi_{i \ell} P_{\ell} \\
+A_{\mathbf{p} i}^{T} A_{\mathbf{p} i}+\sum_{\nu=1}^{R}\left(B_{\mathbf{p} i} K_{i \nu}\right)^{T}\left(B_{\mathbf{p} i} K_{i \nu}\right)+2 P_{i} P_{i}<0 ; j<k ; j, k=1,2, \ldots, R .
\end{gathered}
$$

Prova: Considere que o SFSM (4.5) com $w \equiv 0$ no instante $t$ esteja no modo $i$, isto é, $r=i, i \in \mathbb{S}$. Também considere a função de Lyapunov candidata (4.18) e seu operador infinitesimal fraco ao longo da trajetória do sistema (4.19). Substituindo (4.5) com $w \equiv 0$ em (4.19) e usando o fato de que $m_{i}(z)=1$, quando $r=i$, resulta

$$
\begin{aligned}
\mathcal{A} V(x, i)= & x^{T}\left[\sum_{j=1}^{R} n_{i j}^{2}(x)\left(G_{i j}^{T} P_{i}+P_{i} G_{i j}\right)+2 \sum_{j<k}^{R} n_{i j}(x) n_{i k}(x)\left(H_{i j k}^{T} P_{i}+P_{i} H_{i j k}\right)\right. \\
& \left.+\sum_{\ell=1}^{N} \pi_{i \ell} P_{i}\right] x+\Delta f^{T} P_{i} x+x^{T} P_{i} \Delta f+\Delta g^{T} P_{i} x+x^{T} P_{i} \Delta g
\end{aligned}
$$

Usando $\pi_{i}=-\pi_{i i}=\sum_{j=1, j \neq i}^{N} \pi_{i j}$, pode-se escrever (4.22) como segue

$$
\begin{aligned}
\mathcal{A} V(x, i)= & x^{T}\left\{\sum_{j=1}^{R} n_{i j}^{2}(x)\left[\left(G_{i j}^{T}-\frac{1}{4} \pi_{i} \mathbf{I}\right) P_{i}+P_{i}\left(G_{i j}-\frac{1}{4} \pi_{i} \mathbf{I}\right)\right]\right. \\
& +2 \sum_{j<k}^{R} n_{i j}(x) n_{i k}(x)\left[\left(H_{i j k}-\frac{1}{4} \pi_{i} \mathbf{I}\right)^{T} P_{i}+P_{i}\left(H_{i j k}-\frac{1}{4} \pi_{i} \mathbf{I}\right)\right] \\
& \left.+\sum_{\ell=1 ; \ell \neq i}^{N} \pi_{i \ell} P_{i}\right\} x+\Delta f^{T} P_{i} x+x^{T} P_{i} \Delta f+\Delta g^{T} P_{i} x+x^{T} P_{i} \Delta g .
\end{aligned}
$$

Tendo em vista que

$$
\begin{gathered}
\Delta f^{T} P_{i} x+x^{T} P_{i} \Delta f \leq \Delta f^{T} \Delta f+x^{T} P_{i} P_{i} x \\
\Delta g^{T} P_{i} x+x^{T} P_{i} \Delta g \leq \Delta g^{T} \Delta g+x^{T} P_{i} P_{i} x
\end{gathered}
$$


o operador infinitesimal fraco dado por (4.23) satisfaz

$$
\begin{aligned}
\mathcal{A} V(x, i) \leq & x^{T}\left\{\sum_{j=1}^{R} n_{i j}^{2}(x)\left[\left(G_{i j}^{T}-\frac{1}{4} \pi_{i} \mathbf{I}\right) P_{i}+P_{i}\left(G_{i j}-\frac{1}{4} \pi_{i} \mathbf{I}\right)\right]\right. \\
& +2 \sum_{j<k}^{R} n_{i j}(x) n_{i k}(x)\left[\left(H_{i j k}-\frac{1}{4} \pi_{i} \mathbf{I}\right)^{T} P_{i}+P_{i}\left(H_{i j k}-\frac{1}{4} \pi_{i} \mathbf{I}\right)\right] \\
& \left.+\sum_{\ell=1 ; \ell \neq i}^{N} \pi_{i \ell} P_{i}\right\} x+\Delta f^{T} \Delta f+\Delta g^{T} \Delta g+2 x^{T} P_{i} P_{i} x .
\end{aligned}
$$

Agora, substituindo (4.12) e (4.14) em (4.24), obtém-se

$$
\begin{aligned}
\mathcal{A} V(x, i)< & x^{T}\left\{\sum_{j=1}^{R} n_{i j}^{2}(x)\left[\left(G_{i j}-\frac{1}{4} \pi_{i} \mathbf{I}\right)^{T} P_{i}+P_{i}\left(G_{i j}-\frac{1}{4} \pi_{i} \mathbf{I}\right)\right]\right. \\
& +2 \sum_{j<k}^{R} n_{i j}(x) n_{i k}(x)\left[\left(H_{i j k}-\frac{1}{4} \pi_{i} \mathbf{I}\right)^{T} P_{i}+P_{i}\left(H_{i j k}-\frac{1}{4} \pi_{i} \mathbf{I}\right)\right] \\
& \left.+\sum_{\ell=1 ; \ell \neq i}^{N} \pi_{i \ell} P_{\ell}+A_{\mathbf{p} i}^{T} A_{\mathbf{p} i}+\sum_{\nu=1}^{R}\left(B_{\mathbf{p} i} K_{i \nu}\right)^{T}\left(B_{\mathbf{p} i} K_{i \nu}\right)+2 P_{i} P_{i}\right\} x .
\end{aligned}
$$

Se as desigualdades (4.20) e (4.21) são satisfeitas, tem-se $\mathcal{A} V(x, i)<0$ for $x \neq 0$.

Agora, definindo

$$
\begin{gathered}
\mathcal{L}\left(\bar{G}_{i j}, \bar{H}_{i j k}, P_{i}\right):=\left(\bar{G}_{i j}^{T} P_{i}+P_{i} \bar{G}_{i j}\right)+2\left(\bar{H}_{i j k}^{T} P_{i}+P_{i} \bar{H}_{i j k}\right)+\sum_{\ell=1}^{N} \pi_{i \ell} P_{\ell} \\
+A_{\mathbf{p} i}^{T} A_{\mathbf{p} i}+\sum_{\nu=1}^{R}\left(B_{\mathbf{p} i} K_{i \nu}\right)^{T}\left(B_{\mathbf{p} i} K_{i \nu}\right)+2 P_{i} P_{i}
\end{gathered}
$$

com $\bar{G}_{i j}=\sum_{j=1}^{R} n_{i j}^{2}(x) G_{i j}$ e $\bar{H}_{i j k}=\sum_{j<k}^{R} n_{i j}(x) n_{i k}(x) H_{i j k}$ e substituindo (4.26) em (4.25), pode-se escrever

$$
\mathcal{A} V(x, i)<x^{T} \mathcal{L}\left(\bar{G}_{i j}, \bar{H}_{i j k}, P_{i}\right) x .
$$

Desta forma, para todo $x \neq 0$ e $i \in \mathbb{S}$, tem-se

$$
\begin{aligned}
\frac{\mathcal{A} V(x, i)}{V(x, i)} & <\frac{x^{T} \mathcal{L}\left(\bar{G}_{i j}, \bar{H}_{i j k}, P_{i}\right) x}{x^{T} P_{i} x} \\
& \leq-\rho
\end{aligned}
$$


onde $\rho$ é uma constante positiva definida por

$$
\rho=\min _{i \in \mathbb{S}}\left\{\frac{\lambda_{\min }\left[-\mathcal{L}\left(\bar{G}_{i j}, \bar{H}_{i j k}, P_{i}\right)\right]}{\lambda_{\max }\left[P_{i}\right]}\right\} .
$$

Novamente, pela fórmula de Dynkin (Kushner, 1967), tem-se

$$
E[V(x(t), r(t))]-V\left(x_{0}, r_{0}\right)=E\left[\int_{0}^{t} \mathcal{A} V(x(s), r(s)) d s\right]
$$

Então, substitutindo (4.28) em (4.30), obtém-se

$$
\begin{aligned}
E[V(x(t), r(t))]-V\left(x_{0}, r_{0}\right) & \leq E\left[\int_{0}^{t}-\rho V(x(s), r(s)) d s\right] \\
& =-\rho \int_{0}^{t} E[V(x(s), r(s)) d s]
\end{aligned}
$$

Usando o lema de Gronwall-Bellman in (4.31), tem-se

$$
E[V(x(t), r(t))] \leq V\left(x_{0}, r_{0}\right) e^{-\rho t} .
$$

Integrando ambos os lados de (4.32) e tomando o limite quando $T \rightarrow \infty$, resulta

$$
\begin{aligned}
\lim _{T \rightarrow \infty} E\left[\int_{0}^{T} x^{T} P_{i} x d t \mid x_{0}, r_{0}\right] & \leq \frac{1}{\rho} x_{0}^{T} P_{i} x_{0} \\
& \leq \frac{1}{\rho} \lambda_{\max }\left[P_{i}\right] x_{0}^{T} x_{0}
\end{aligned}
$$

Considerando o fato de que em (4.33) $P_{i}=P_{i}^{T}, P_{i}>0$ para todo $i \in \mathbb{S}$, o resultado segue pela Definição 7 .

Observação 7 As condições de estabilidade estocástica da Proposição 9 correspondem àquelas da Proposição 4 aumentadas para incluir os erros de modelagem descritos em forma de (4.10) e (4.11).

Proposição 10 Sejam $x_{0}$ e $r_{0}$ o vetor de condições iniciais e o modo inicial do sistema, respectivamente. O SFSM (4.2) com lei de controlefuzzy (4.3) tem desempenho $\mathcal{H}_{\infty}$ (4.16) para todas as incertezas na modelagem admissiveis, se existe um conjunto 
de matrizes $P_{i}=P_{i}^{T}, P_{i}>0$ de dimensões apropriadas satisfazendo $\forall i \in \mathbb{S}$

$$
\begin{gathered}
\left(G_{i j}-\frac{1}{4} \pi_{i} \mathbf{I}\right)^{T} P_{i}+P_{i}\left(G_{i j}-\frac{1}{4} \pi_{i} \mathbf{I}\right)+\sum_{\ell=1 ; \ell \neq i}^{N} \pi_{i \ell} P_{\ell}+A_{\mathbf{p} i}^{T} A_{\mathbf{p} i} \\
+\sum_{\nu=1}^{R}\left(B_{\mathbf{p} i} K_{i \nu}\right)^{T}\left(B_{\mathbf{p} i} K_{i \nu}\right)+\left(2+\frac{1}{\gamma^{2}}\right) P_{i} P_{i}+S_{i}<0 ; j=1,2, \ldots, R \\
\left(H_{i j k}-\frac{1}{4} \pi_{i} \mathbf{I}\right)^{T} P_{i}+P_{i}\left(H_{i j k}-\frac{1}{4} \pi_{i} \mathbf{I}\right)+\sum_{\ell=1 ; \ell \neq i}^{N} \pi_{i \ell} P_{\ell}+A_{\mathbf{p} i}^{T} A_{\mathbf{p} i} \\
+\sum_{\nu=1}^{R}\left(B_{\mathbf{p} i} K_{i \nu}\right)^{T}\left(B_{\mathbf{p} i} K_{i \nu}\right)+\left(2+\frac{1}{\gamma^{2}}\right) P_{i} P_{i}+S_{i}<0 ; j<k ; j, k=1,2, \ldots, R(
\end{gathered}
$$

Prova: Seja $i$ o modo do sistema no instante $t$, que é, $r=i, i \in \mathbb{S}$. Considere o SFSM em malha-fechada (4.5). Escolhendo a função de Lyapunov candidata como em (4.18), substituindo (4.5) in (4.19) e procedendo de maneira similar como na prova da Proposição 9, obtém-se

$$
\begin{aligned}
\mathcal{A} V(x, i)< & x^{T}\left\{\sum_{j=1}^{R} n_{i j}^{2}(x)\left[\left(G_{i j}-\frac{1}{4} \pi_{i} \mathbf{I}\right)^{T} P_{i}+P_{i}\left(G_{i j}-\frac{1}{4} \pi_{i} \mathbf{I}\right)\right]\right. \\
& +2 \sum_{j<k}^{R} n_{i j}(x) n_{i k}(x)\left[\left(H_{i j k}-\frac{1}{4} \pi_{i} \mathbf{I}\right)^{T} P_{i}+P_{i}\left(H_{i j k}-\frac{1}{4} \pi_{i} \mathbf{I}\right)\right] \\
& \left.+\sum_{\ell=1 ; \ell \neq i}^{N} \pi_{i \ell} P_{\ell}+A_{\mathbf{p} i}^{T} A_{\mathbf{p} i}+\sum_{\nu=1}^{R}\left(B_{\mathbf{p} i} K_{i \nu}\right)^{T}\left(B_{\mathbf{p} i} K_{i \nu}\right)+2 P_{i} P_{i}\right\} x \\
& +x^{T} P_{i} w+w^{T} P_{i} x .
\end{aligned}
$$

A desigualdade (4.36) pode ser escrita como

$$
\begin{aligned}
\mathcal{A} V(x, i)< & x^{T}\left\{\sum_{j=1}^{R} n_{i j}^{2}(x)\left[\left(G_{i j}-\frac{1}{4} \pi_{i} \mathbf{I}\right)^{T} P_{i}+P_{i}\left(G_{i j}-\frac{1}{4} \pi_{i} \mathbf{I}\right)\right]\right. \\
& +2 \sum_{j<k}^{R} n_{i j}(x) n_{i k}(x)\left[\left(H_{i j k}-\frac{1}{4} \pi_{i} \mathbf{I}\right)^{T} P_{i}+P_{i}\left(H_{i j k}-\frac{1}{4} \pi_{i} \mathbf{I}\right)\right] \\
& \left.+\sum_{\ell=1 ; \ell \neq i}^{N} \pi_{i \ell} P_{\ell}+A_{\mathbf{p} i}^{T} A_{\mathbf{p} i}+\sum_{\nu=1}^{R}\left(B_{\mathbf{p} i} K_{i \nu}\right)^{T}\left(B_{\mathbf{p} i} K_{i \nu}\right)+2 P_{i} P_{i}\right\}
\end{aligned}
$$




$$
\begin{aligned}
& +\left(x^{T} P_{i} w+w^{T} P_{i} x-\gamma^{2} w^{T} w-\frac{1}{\gamma^{2}} x^{T} P_{i} P_{i} x\right)+\gamma^{2} w^{T} w+\frac{1}{\gamma^{2}} x^{T} P_{i} P_{i} x \\
= & x^{T}\left\{\sum_{j=1}^{R} n_{i j}^{2}(x)\left[\left(G_{i j}-\frac{1}{4} \pi_{i} \mathbf{I}\right)^{T} P_{i}+P_{i}\left(G_{i j}-\frac{1}{4} \pi_{i} \mathbf{I}\right)\right]\right. \\
& +2 \sum_{j<k}^{R} n_{i j}(x) n_{i k}(x)\left[\left(H_{i j k}-\frac{1}{4} \pi_{i} \mathbf{I}\right)^{T} P_{i}+P_{i}\left(H_{i j k}-\frac{1}{4} \pi_{i} \mathbf{I}\right)\right] \\
& \left.+\sum_{\ell=1 ; \ell \neq i}^{N} \pi_{i \ell} P_{\ell}+A_{\mathbf{p} i}^{T} A_{\mathbf{p} i}+\sum_{\nu=1}^{R}\left(B_{\mathbf{p} i} K_{i \nu}\right)^{T}\left(B_{\mathbf{p} i} K_{i \nu}\right)+2 P_{i} P_{i}\right\} x \\
& -\left(\frac{1}{\gamma} P_{i} x-\gamma w\right)^{T}\left(\frac{1}{\gamma} P_{i} x-\gamma w\right)+\gamma^{2} w^{T} w+\frac{1}{\gamma^{2}} x^{T} P_{i} P_{i} x \\
< & x^{T}\left\{\sum_{j=1}^{R} n_{i j}^{2}(x)\left[\left(G_{i j}-\frac{1}{4} \pi_{i} \mathbf{I}\right)^{T} P_{i}+P_{i}\left(G_{i j}-\frac{1}{4} \pi_{i} \mathbf{I}\right)\right]\right. \\
& +2 \sum_{j<k}^{R} n_{i j}(x) n_{i k}(x)\left[\left(H_{i j k}-\frac{1}{4} \pi_{i} \mathbf{I}\right)^{T} P_{i}+P_{i}\left(H_{i j k}-\frac{1}{4} \pi_{i} \mathbf{I}\right)\right] \\
& \left.+\sum_{\ell=1 ; \ell \neq i}^{N} \pi_{i \ell} P_{\ell}+A_{\mathbf{p} i}^{T} A_{\mathbf{p} i}+\sum_{\nu=1}^{R}\left(B_{\mathbf{p} i} K_{i \nu}\right)^{T}\left(B_{\mathbf{p} i} K_{i \nu}\right)+2 P_{i} P_{i}\right\} x \\
+ & \gamma^{2} w^{T} w+\frac{1}{\gamma^{2}} x^{T} P_{i} P_{i} x .
\end{aligned}
$$

Agora, considere que exista um conjunto de matrizes $P_{i}=P_{i}^{T}, P_{i}>0, i \in \mathbb{S}$ satisfazendo (4.34) e (4.35). Multiplicando (4.34) por $n_{i j}^{2}(x)$ e (4.35) por $2 n_{i j}(x) n_{i k}(x)$, obtém-se

$$
\begin{gathered}
\sum_{j=1}^{R} n_{i j}^{2}(x)\left[\left(G_{i j}-\frac{1}{4} \pi_{i} \mathbf{I}\right)^{T} P_{i}+P_{i}\left(G_{i j}-\frac{1}{4} \pi_{i} \mathbf{I}\right)\right]+\sum_{j=1}^{R} n_{i j}^{2}(x)\left(\sum_{\ell=1 ; \ell \neq i}^{N} \pi_{i \ell} P_{\ell}+A_{\mathbf{p} i}^{T} A_{\mathbf{p} i}\right) \\
+\sum_{j=1}^{R} n_{i j}^{2}(x)\left[\sum_{\nu=1}^{R}\left(B_{\mathbf{p} i} K_{i \nu}\right)^{T}\left(B_{\mathbf{p} i} K_{i \nu}\right)+2 P_{i} P_{i}\right]<-\sum_{j=1}^{R} n_{i j}^{2}(x)\left(S_{i}+\frac{1}{\gamma^{2}} P_{i} P_{i}\right)
\end{gathered}
$$


$\mathrm{e}$

$$
\begin{gathered}
2 \sum_{j<k}^{R} n_{i j}(x) n_{i k}(x)\left[\left(H_{i j k}-\frac{1}{4} \pi_{i} \mathbf{I}\right)^{T} P_{i}+P_{i}\left(H_{i j k}-\frac{1}{4} \pi_{i} \mathbf{I}\right)\right] \\
+2 \sum_{j<k}^{R} n_{i j}(x) n_{i k}(x)\left(\sum_{\ell=1 ; \ell \neq i}^{N} \pi_{i \ell} P_{\ell}+A_{\mathbf{p} i}^{T} A_{\mathbf{p} i}\right) \\
+2 \sum_{j<k}^{R} n_{i j}(x) n_{i k}(x)\left[\sum_{\nu=1}^{R}\left(B_{\mathbf{p} i} K_{i \nu}\right)^{T}\left(B_{\mathbf{p} i} K_{i \nu}\right)+2 P_{i} P_{i}\right] \\
<-2 \sum_{j<k}^{R} n_{i j}(x) n_{i k}(x)\left(S_{i}+\frac{1}{\gamma^{2}} P_{i} P_{i}\right) .
\end{gathered}
$$

Então, adicionando (4.39) a (4.40) e, novamente, usando $\sum_{j=1}^{R} \sum_{k=1}^{R} n_{i j}(x) n_{i k}(x)=$ $\sum_{j=1}^{R} n_{i j}^{2}(x)+2 \sum_{j<k}^{R} n_{i j}(x) n_{i k}(x)=1$, tem-se

$$
\begin{gathered}
\sum_{j=1}^{R} n_{i j}^{2}(x)\left[\left(G_{i j}-\frac{1}{4} \pi_{i} \mathbf{I}\right)^{T} P_{i}+P_{i}\left(G_{i j}-\frac{1}{4} \pi_{i} \mathbf{I}\right)\right] \\
+2 \sum_{j<k}^{R} n_{i j}(x) n_{i k}(x)\left[\left(H_{i j k}-\frac{1}{4} \pi_{i} \mathbf{I}\right)^{T} P_{i}+P_{i}\left(H_{i j k}-\frac{1}{4} \pi_{i} \mathbf{I}\right)\right] \\
+\sum_{\ell=1 ; \ell \neq i}^{N} \pi_{i \ell} P_{\ell}+A_{\mathbf{p} i}^{T} A_{\mathbf{p} i}+\sum_{\nu=1}^{R}\left(B_{\mathbf{p} i} K_{i \nu}\right)^{T}\left(B_{\mathbf{p} i} K_{i \nu}\right)+2 P_{i} P_{i}<-\left(S_{i}+\frac{1}{\gamma^{2}} P_{i} P_{i}\right) .
\end{gathered}
$$

Agora, pré-multiplicando (4.41) por $x^{T}$ e pós-multiplicando $x$, pela Proposição 9, podese verificar que

$$
\begin{aligned}
\mathcal{A} V(x, i) & <-x^{T}\left(S_{i}+\frac{1}{\gamma^{2}} P_{i} P_{i}\right) x+\gamma^{2} w^{T} w+\frac{1}{\gamma^{2}} x^{T} P_{i} P_{i} x \\
& =-x^{T} S_{i} x+\gamma^{2} w^{T} w .
\end{aligned}
$$

Note que, quando o distúrbio $w \equiv 0$ em (4.42), tem-se $\mathcal{A} V(x, i)<0$, que assegura a estabilidade assintótica do SFSM em malha-fechada de acordo com a Proposição 9. Integrando ambos os lados de (4.42), obtém-se

$$
E\left[\int_{0}^{T} \mathcal{A} V(x(t), r(t)) d t\right]+E\left[\int_{0}^{T} x(t)^{T} S_{i} x(t) d t\right]-\gamma^{2} \int_{0}^{T} w(t)^{T} w(t) d t<0
$$


Finalmente, substitutindo (4.30) em (4.43), resulta

$$
E[V(x(T), r(T))]-V\left(x_{0}, r_{0}\right)<-E\left[\int_{0}^{T} x(t)^{T} S_{i} x(t) d t\right]+\gamma^{2} \int_{0}^{T} w(t)^{T} w(t) d t
$$

e o resultado segue pela Definição 8.

Observação 8 Para o caso do distúrbio $w$ limitado por $\|w\| \leq w_{b d}$, usando (4.42), obtém-se $\mathcal{A} V(x, i)<0$, sempre que $\|x\| \geq \gamma w_{b d} / \sqrt{c_{1}}$, com $c_{1}=\lambda_{\min }\left(S_{i}\right), \quad i \in \mathbb{S}$, assim pode ser mostrado que usando a extensão de Lyapunov padrão (Khalil, 1996) a solução do SFSM em malha-fechada (4.5) é ultimamente limitado (ultimately bounded) no sentido da média-quadrática e que o desempenho $\mathcal{H}_{\infty}$ do controlador fuzzy dado por (4.16) é garantido.

Visando obter um procedimento de projeto mais sistemático, reescrevem-se as desigualdades (4.34) e (4.35) como LMI's. Então, dados $G_{i j}=A_{i j}-B_{i j} K_{i j}$ e $H_{i j k}=$ $\frac{1}{2}\left(A_{i j}-B_{i j} K_{i k}+A_{i k}-B_{i k} K_{i j}\right)$, pode-se escrever (4.34) e (4.35) na seguinte forma

$$
\begin{gathered}
A_{i j}^{T} P_{i}+P_{i} A_{i j}-K_{i j}^{T} B_{i j}^{T} P_{i}-P_{i} B_{i j} K_{i j}-\frac{1}{2} \pi_{i} \mathbf{I}+\sum_{\ell=1 ; \ell \neq i}^{N} \pi_{i \ell} P_{\ell}+A_{\mathbf{p} i}^{T} A_{\mathbf{p} i} \\
+\sum_{\nu=1}^{R}\left(B_{\mathbf{p} i} K_{i \nu}\right)^{T}\left(B_{\mathbf{p} i} K_{i \nu}\right)+\left(2+\frac{1}{\gamma^{2}}\right) P_{i} P_{i}+S_{i}<0 ; j=1,2, \ldots, R
\end{gathered}
$$

$\mathrm{e}$

$$
\begin{gathered}
A_{i j}^{T} P_{i}+P_{i} A_{i j}-K_{i k}^{T} B_{i j}^{T} P_{i}-P_{i} B_{i j} K_{i k} \\
+A_{i k}^{T} P_{i}+P_{i} A_{i k}-K_{i j}^{T} B_{i k}^{T} P_{i}-P_{i} B_{i k} K_{i j}-\frac{1}{2} \pi_{i} \mathbf{I}+\sum_{\ell=1 ; \ell \neq i}^{N} \pi_{i \ell} P_{\ell}+A_{\mathbf{p} i}^{T} A_{\mathbf{p} i} \\
+\sum_{\nu=1}^{R}\left(B_{\mathbf{p} i} K_{i \nu}\right)^{T}\left(B_{\mathbf{p} i} K_{i \nu}\right)+\left(2+\frac{1}{\gamma^{2}}\right) P_{i} P_{i}+S_{i}<0 ; j<k ; j, k=1,2, \ldots, R .(4)
\end{gathered}
$$

Novamente, pode ser notado que as desigualdades (4.45) e (4.46) não são LMI's, pois aparecem produtos entre as variáveis $K_{i j}$ e $P_{i}$. No entanto, pré e pós-multiplicando estas desigualdades por $P_{i}^{-1}$ e substituindo (3.29) e (3.30), pode-se obter

$$
\begin{aligned}
X_{i} A_{i j}^{T}+ & A_{i j} X_{i}-Y_{i j}^{T} B_{i j}^{T}-B_{i j} Y_{i j}-\frac{1}{2} \pi_{i} X_{i}+\sum_{\ell=1 ; \ell \neq i}^{N} \pi_{i \ell}\left(X_{i} X_{\ell}^{-1} X_{i}\right)+\left(2+\frac{1}{\gamma^{2}}\right) \mathbf{I} \\
& +X_{i}\left(S_{i}+A_{\mathbf{p} i}^{T} A_{\mathbf{p} i}\right) X_{i}+\sum_{\nu=1}^{R} Y_{i \nu}^{T} B_{\mathbf{p} i}^{T} B_{\mathbf{p} i} Y_{i \nu}<0 ; j=1,2, \ldots, R
\end{aligned}
$$


e

$$
\begin{gathered}
X_{i} A_{i j}^{T}+A_{i j} X_{i}-Y_{i k}^{T} B_{i j}^{T}-B_{i j} Y_{i k} \\
+X_{i} A_{i k}^{T}+A_{i k} X_{i}-Y_{i j}^{T} B_{i k}^{T}-B_{i k} Y_{i j}-\frac{1}{2} \pi_{i} X_{i}+\sum_{\ell=1 ; \ell \neq i}^{N} \pi_{i \ell}\left(X_{i} X_{\ell}^{-1} X_{i}\right)+\left(2+\frac{1}{\gamma^{2}}\right) \mathbf{I} \\
+X_{i}\left(S_{i}+A_{\mathbf{p} i}^{T} A_{\mathbf{p} i}\right) X_{i}+\sum_{\nu=1}^{R} Y_{i \nu}^{T} B_{\mathbf{p} i}^{T} B_{\mathbf{p} i} Y_{i \nu}<0 ; j<k ; j, k=1,2, \ldots, R
\end{gathered}
$$

onde $X_{i}:=P_{i}^{-1}$ e $Y_{i j}:=K_{i j} X_{i}$. Agora, definindo

$$
\begin{aligned}
& \hat{T}_{i j}:=\left[\begin{array}{cccccc}
T_{i j}+2 \mathbf{I} & 1 & X_{i} & B_{\mathbf{p} i} Y_{i 1} & \cdots & B_{\mathbf{p} i} Y_{i R} \\
1 & -\gamma^{2} \mathbf{I} & \mathbf{0} & \mathbf{0} & \cdots & \mathbf{0} \\
X_{i} & \mathbf{0} & -\left(S_{i}+A_{\mathbf{p} i}^{T} A_{\mathbf{p} i}\right)^{-1} & \mathbf{0} & \cdots & \mathbf{0} \\
\left(B_{\mathbf{p} i} Y_{i 1}\right)^{T} & \mathbf{0} & \mathbf{0} & -\mathbf{I} & \cdots & \mathbf{0} \\
\vdots & \vdots & \vdots & \vdots & \vdots & \vdots \\
\left(B_{\mathbf{p} i} Y_{i R}\right)^{T} & \mathbf{0} & \mathbf{0} & \mathbf{0} & \cdots & -\mathbf{I}
\end{array}\right] \\
& \hat{U}_{i j k}:=\left[\begin{array}{cccccc}
U_{i j k}+2 \mathbf{I} & 1 & X_{i} & B_{\mathbf{p} i} Y_{i 1} & \cdots & B_{\mathbf{p} i} Y_{i R} \\
1 & -\gamma^{2} \mathbf{I} & \mathbf{0} & \mathbf{0} & \cdots & \mathbf{0} \\
X_{i} & \mathbf{0} & -\left(S_{i}+A_{\mathbf{p} i}^{T} A_{\mathbf{p} i}\right)^{-1} & \mathbf{0} & \cdots & \mathbf{0} \\
\left(B_{\mathbf{p} i} Y_{i 1}\right)^{T} & \mathbf{0} & \mathbf{0} & -\mathbf{I} & \cdots & \mathbf{0} \\
\vdots & \vdots & \vdots & \vdots & \vdots & \vdots \\
\left(B_{\mathbf{p} i} Y_{i R}\right)^{T} & \mathbf{0} & \mathbf{0} & \mathbf{0} & \cdots & -\mathbf{I}
\end{array}\right] \\
& Z_{i}:=\left[\begin{array}{llllll}
\pi_{i 1}^{1 / 2} X_{i} & \ldots & \pi_{i i-1}^{1 / 2} X_{i} & \pi_{i i+1}^{1 / 2} X_{i} & \ldots & \pi_{i N}^{1 / 2} X_{i}
\end{array}\right] \\
& W_{i}:=\operatorname{diag}\left\{\begin{array}{llllll}
X_{1} & \ldots & X_{i-1} & X_{i+1} & \ldots & X_{N}
\end{array}\right\}
\end{aligned}
$$

com

$$
\begin{aligned}
T_{i j}:= & X_{i} A_{i j}^{T}+A_{i j} X_{i}-Y_{i j}^{T} B_{i j}^{T}-B_{i j} Y_{i j}-\frac{1}{2} \pi_{i} X_{i} \\
U_{i j k}:= & X_{i} A_{i j}^{T}+A_{i j} X_{i}-Y_{i k}^{T} B_{i j}^{T}-B_{i j} Y_{i k} \\
& +X_{i} A_{i k}^{T}+A_{i k} X_{i}-Y_{i j}^{T} B_{i k}^{T}-B_{i k} Y_{i j}-\frac{1}{2} \pi_{i} X_{i}
\end{aligned}
$$


e usando os complementos de Schur, chega-se às seguintes desigualdades matriciais

$$
\left[\begin{array}{ll}
\hat{T}_{i j} & Z_{i} \\
Z_{i}^{T} & -W_{i}
\end{array}\right]<0 ; j=1,2, \ldots, R
$$

e

$$
\left[\begin{array}{ll}
\hat{U}_{i j k} & Z_{i} \\
Z_{i}^{T} & -W_{i}
\end{array}\right]<0 ; j<k ; j, k=1,2, \ldots, R
$$

Usando a Proposição 10 e as LMI's (4.53) e (4.54), formula-se o seguinte problema de otimização para o projeto de controle fuzzy estabilizante com desempenho $\mathcal{H}_{\infty}$.

Problema 4 Encontrar um conjunto de matrizes $X_{i}=X_{i}^{T}, X_{i}>0$ e um conjunto de matrizes $Y_{i j}$ de dimensões apropriadas minimizando $\gamma^{2}$ sujeito a

$$
X_{i}>0,(4.53) \text { e (4.54) } \forall i \in \mathbb{S} .
$$

Se o Problema 4 for factível, pode-se obter os ganhos de realimentação de estado usando $K_{i j}=Y_{i j} X_{i}^{-1}, i \in \mathbb{S}, j=1,2, \ldots, R$.

Observação 9 Nas abordagens de estabilização estocástica apresentadas, os projetos de controle fuzzy formulados em LMI's são baseados nas matrizes $\left(A_{i j}, B_{i j}, \Pi\right), i \in \mathbb{S}$, $j=1,2, \ldots, R$. Desta forma, o grau de complexidade de busca por uma solução está fortemente relacionado ao número de regras de inferência e de modos assumidos pelo processo de Markov. Uma alternativa para flexibilizar ou relaxar o projeto é explorar as propriedades das funções de pertinência normalizadas, assim como no caso do projeto de controle fuzzy TS (Teixeira et al., 2003; Teixeira et al., 2000; Tanaka et al., 1998).

Observação 10 Tendo em vista o projeto de controle fuzzy para o caso em que o estado de equilíbrio não é a origem, i.e., $(x, u) \neq 0$, deve-se fazer uma mudança de coordenadas, tornando a origem um estado de equilíbrio, antes de projetar o controlador. 


\section{Capítulo 5}

\section{Representação de um Sistema}

\section{Elétrico de Potência por um}

\section{Sistema com Saltos Markovianos}

\subsection{Considerações Iniciais}

A energia elétrica por apresentar maior facilidade de manipulação, tanto para transmissão quanto para processamento, é a forma de energia mais utilizada atualmente e está dificilmente disponível na forma elétrica, sendo convertida para esta forma na entrada de um sistema e posteriormente revertida para forma não elétrica na saída do mesmo. Deste modo, a energia elétrica desde a sua captação até sua entrega aos consumidores sofre manipulações em diversos equipamentos. Denomina-se SEP ao conjunto responsável pela geração, transmissão e distribuição da energia elétrica. A geração de energia elétrica pode ser realizada via a energia potencial da água (geração hidrelétrica), via a energia potencial dos combustíveis (geração termoelétrica), etc. A parte correspondente à transmissão compreende o transporte da energia elétrica gerada até os centros consumidores, onde os níveis de tensão são padronizados em valores elevados em função da potência a ser transmitida e das distâncias aos centros de consumo a fim de proporcionar a sua viabilidade econômica. A parte final na transferência de potência aos pequenos e médios consumidores é o que se chama de distribuição. A distribuição é a parte do SEP interna aos centros de utilização, iniciando nas subestações abaixadoras, onde a 
tensão da linha de transmissão é rebaixada a valores padronizados, também em alta tensão, para atender as redes de distribuição primária. Portanto, a função primordial de um SEP é converter a energia de uma das formas naturalmente disponíveis para a forma elétrica, transportando-a até os pontos de consumo (Creder, 1995; Fitzgerald e Kingsley, 1975).

O projeto e a operação de SEP's deve satisfazer a demanda da carga (tanto ativa, quanto reativa), que é continuamente variável, diferindo assim das demais formas de energia, visto que a eletricidade não pode ser convencionalmente armazenada em quantidades suficientes. Em geral, uma reserva girante de potência deve ser mantida e controlada apropriadamente a todo instante e o fornecimento da energia deve ser projetado de forma a minimizar tanto os custos, quanto o impacto ecológico, e, principalmente, oferecer qualidade no fornecimento, atendendo padrões de requisitos mínimos relacionados aos fatores de tensões e freqüências constantes e bom nível de confiabilidade. Sendo assim, a potência elétrica produzida nas centrais geradoras é transmitida aos consumidores por uma rede muito complexa de elementos individuais, tais como, linhas de transmissão, transformadores e dispositivos de chaveamento, dentre outros, ficando o sistema como um todo constituído por múltiplas unidades geradoras e diversas unidades de transformadores, que introduzem redundâncias na estrutura do SEP, visando garantir uma certa robustez diante de situações de contingência não usuais, sem que haja a interrupção do fornecimento de energia elétrica ao consumidor.

\subsubsection{Tipos de SEP's}

Existem basicamente dois tipos de SEP's: sistemas de potência DC (corrente direta) e sistemas de potência AC (corrente alternada). O primeiro sistema de potência DC surgiu em 1882, consecutivamente ao seu desenvolvimento, surgiram as primeiras limitações, tais como, elevadas perdas em transmissões, grandes quedas de tensões, dentre outras. Logo, fez-se necessário a transformação de tensões. Daí surgiram os sistemas de potência AC monofásicos, que mais tarde deram origem aos sistemas AC polifásicos.

Na virada do século XX, os sistemas de potência AC prevaleceram sobre os DC, visto que neste tipo de sistema os níveis de tensão podem ser facilmente transformados, tornando flexível o uso de diferentes tensões para geração, transmissão e consumo. Outra vantagem encontrada foi a utilização das máquinas AC, tais como, motores e ge- 
radores, que são mais simples e financeiramente mais econômicas que as máquinas DC. Atualmente, os SEP's possuem diversas estruturas e tamanhos, apresentando porém características básicas, tais como, o fato de serem constituídos de sistemas AC trifásicos, operando essencialmente com tensão constante, também utilizando máquinas síncronas para geração de eletricidade, bem como fazendo a transmissão com potências significativas para atender consumidores espalhados em grandes áreas geográficas. Estas características fazem com que o sistema de transmissão seja composto por vários subsistemas operando em níveis de tensões diferentes.

\subsubsection{Operação de SEP's}

A operação de SEP's está sujeita a uma grande variedade de distúrbios ocasionados por fatores externos ou internos, tais como, quedas de linhas de transmissão, variações de carga, falhas em equipamentos, fenômenos da natureza, dentre outros. Tais fatores tornam muito complexa a operação dos SEP's, principalmente nas ações de controle, além de provocarem mudanças repentinas na sua estrutura. O grau de confiabilidade na operação de um SEP é considerado uma importante especificação de projeto que deve ser atendida mesmo na presença de grandes distúrbios (Wang et al., 1993; Wang et al., 1992).

Nas últimas décadas, o interesse por problemas relacionados à qualidade de energia de SEP's vem aumentando, principalmente entre concessionárias e consumidores. O termo qualidade de energia pode apresentar várias definições completamente diferentes, dependendo da ênfase adotada. Por exemplo, as concessionárias de energia elétrica podem associar a qualidade de energia à confiabilidade e às estatísticas de continuidade de operação do sistema. Os fabricantes de equipamentos podem definir qualidade de energia em relação aos requisitos de suprimento de energia, de modo que os equipamentos trabalhem corretamente, podendo este conceito ser diferente de fabricante para fabricante. Porém, em última instância, devem prevalecer os interesses de qualidade de energia dos consumidores. No entanto, o conceito de qualidade de energia é bem mais amplo e se refere a qualquer problema ocorrido em um SEP que se manifeste na tensão, na corrente, ou na freqüência e que resulte em falha de operação de equipamentos (Dugan et al., 1996).

A manutenção do índice de qualidade de tensão, por exemplo, é muito importante 


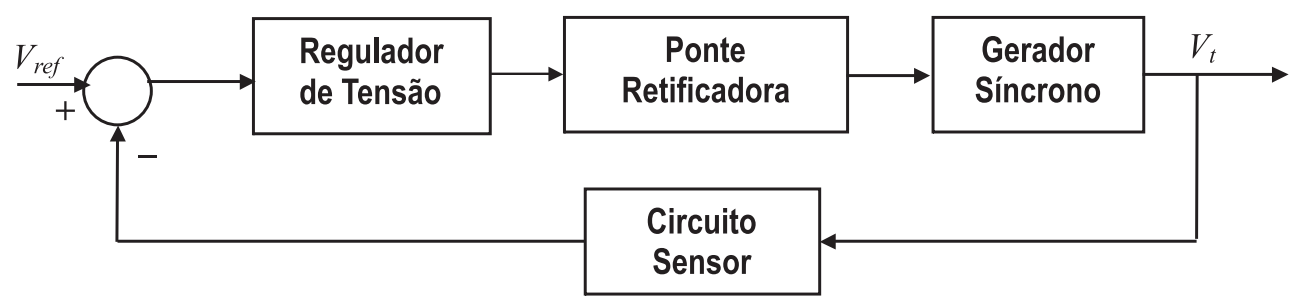

Figura 5.1: Estrutura básica de um controle do sistema de excitação.

para fontes de potência na operação de um SEP, visto que, tanto os equipamentos das concessionárias de energia elétrica, quanto os equipamentos dos consumidores são projetados para operar dentro de uma certa faixa de tensão. A operação prolongada do equipamento em tensões fora da faixa permitida pode afetar adversamente seu desempenho e, possivelmente, causar-lhe danos. O sistema de excitação do gerador síncrono é a parte mais importante de um SEP para o controle de tensão. Em geral, o sistema de controle da excitação de um gerador consiste de um regulador de tensão e de circuitos de sensores, limitadores e proteção. Sendo a principal função deste sistema, regular, tão rápido quanto possível, a excitação do gerador na ocorrência de faltas e assegurar que seus limites de capacidade não sejam ultrapassados (Zhu et al., 1997). A Figura 5.1 mostra a estrutura básica de controle do sistema de excitação de um gerador síncrono.

Outro problema de qualidade de energia é a qualidade na freqüência da rede elétrica. Mudanças na freqüência da rede elétrica são ocasionadas pela perda de sincronismo no SEP depois de uma falta repentina. As seguintes medidas de prevenção contra perdas de sincronismo em um SEP podem ser adotadas durante o projeto de controle (Wang et al., 1993):

- utilizar uma resistência nos terminais do gerador, com a intenção de corrigir o desbalanceamento entre a potência gerada e a potência entreque pelo gerador;

- utilizar capacitâncias em série, visando aumentar a capacidade de transferência de potência de uma linha de transmissão através de uma redução na reatância da rede nos terminais da linha;

- aumentar rapidamente a excitação do gerador;

- introduzir disjuntores e dispositivos de acionamento rápidos, para minimizar o efeito de faltas pela operação de disjuntores independentes; 
- diminuir rapidamente a potência mecânica entregue pela turbina.

A perda de sincronismo em um SEP, dentre outros fatores, é a principal responsável pelo problema de perda de estabilidade transitória. Entende-se como estabilidade transitória, a manutenção de sincronismo entre os geradores síncronos que compõem um SEP após um distúrbio severo. Assim como a regulação da tensão terminal de um gerador síncrono, o controle no sistema de excitação também permite a manutenção de estabilidade transitória em um SEP (Guo et al., 2001). Em síntese, o sistema de excitação de um gerador síncrono é o principal meio de atuação, tanto para regulação de tensão, quanto para manutenção de estabilidade transitória de um SEP.

\subsection{Modelagem de um SEP em Esquema de Co-Geração}

Considere o sistema de potência máquina-barramento-infinito (MBI) em esquema de co-geração, como mostrado na Figura 5.2, composto por um gerador síncrono, cujo barramento terminal é acoplado por uma linha de transmissão a um equivalente do restante do sistema, o chamado barramento-infinito e, também, ao barramento de cargas do sistema. Considere também que o sistema de excitação do gerador é controlado por um regulador automático de tensão coordenado com um estabilizador de potência. As equações da dinâmica do sistema de potência MBI em esquema de co-geração podem ser obtidas do modelo de Park clássico de uma máquina síncrona (Demello e Concordia, 1969), adotando condições balanceadas e desprezando as resistências dos enrolamento do estator e dos amortecedores, as tensões devidas às derivações do fluxo magnético, os efeitos de saturação e os desvios de freqüência nos termos de tensão e velocidade da máquina síncrona. Em adição, a resistência da linha de transmissão é desprezada. Tendo em vista as simplificações mencionadas, o sistema fica descrito por um conjunto de equações diferenciais e por um conjunto de equações algébricas, que são descritas a seguir.

Equações diferenciais do gerador:

$$
\begin{aligned}
\dot{\delta} & =\omega_{0} \omega \\
\dot{\omega} & =\frac{1}{2 H}\left[P_{m}-E_{q}^{\prime} I_{q}\right] \\
\dot{E}_{q}^{\prime} & =\frac{1}{\tau_{d o}^{\prime}}\left[E_{f d}-E_{q}^{\prime}+\left(x_{d}-x_{d}^{\prime}\right) I_{d}\right]
\end{aligned}
$$




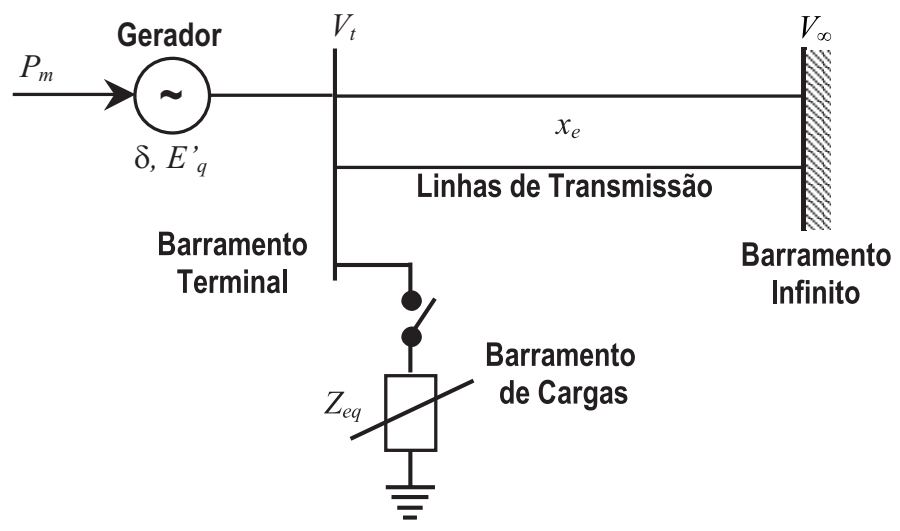

Figura 5.2: Diagrama unifilar do sistema de potência MBI em esquema de co-geração.

Equação diferencial do regulador de tensão:

$$
\dot{E}_{f d}=\frac{1}{T_{e}}\left[K_{e}\left(V_{r e f}-\left|V_{t}\right|+V_{s}\right)-E_{f d}\right]
$$

Equações algébricas do estator do gerador:

$$
\begin{aligned}
V_{q} & =E_{q}^{\prime}+x_{d}^{\prime} I_{d} \\
V_{d} & =-x_{d}^{\prime} I_{q}
\end{aligned}
$$

Outras relações importantes para descrição do sistema são:

$$
\begin{aligned}
\left|V_{t}\right| & =\left(V_{q}^{2}+V_{d}^{2}\right)^{1 / 2} \\
P & =E_{q}^{\prime} I_{q} \\
Q & =-E_{q}^{\prime} I_{d}-x_{d}^{\prime}\left(I_{q}^{2}+I_{d}^{2}\right)
\end{aligned}
$$

Em (5.1) - (5.9), as variáveis e parâmetros envolvidos são listados abaixo:

$\delta$ é o ângulo de potência do gerador [rad];

$\omega$ é a velocidade do rotor do gerador $[\mathrm{rad} / \mathrm{s}]$;

$\omega_{0}$ é a velocidade da máquina síncrona $[\mathrm{rad} / \mathrm{s}] ;$

$P_{m}$ é a potência mecânica de entrada [p.u.];

$P$ é a potência elétrica ativa entregue pelo gerador [p.u.];

$Q$ é a potência elétrica reativa entregue pelo gerador [p.u.]; 
$E_{q}^{\prime}$ é a FEM transitória no eixo em quadratura do gerador [p.u.];

$E_{f d}$ é a FEM equivalente no núcleo de excitação [p.u.];

$I_{q}$ é a corrente no eixo em quadratura [p.u.];

$I_{d}$ é a corrente no eixo direto [p.u.];

$V_{q}$ é a tensão no eixo em quadratura [p.u.];

$V_{d}$ é a tensão no eixo direto [p.u.];

$H$ é a constante de inércia [p.u.];

$\tau_{d o}^{\prime}$ é a constante de tempo do transitório no eixo direto do circuito aberto [s];

$T_{e}$ é a constante de tempo do regulador de tensão [s];

$K_{e}$ é o ganho do regulador de tensão;

$V_{\text {ref }}$ é a tensão de referência do regulador de tensão [p.u.];

$V_{t}$ é a tensão terminal do gerador [p.u.];

$V_{s}$ é a tensão estabilizante [p.u.];

$x_{d}^{\prime}$ é a reatância transitória no eixo direto [p.u.];

$x_{d}$ é a reatância no eixo direto [p.u.];

$x_{e}$ é a reatância equivalente do circuito externo [p.u.].

O sistema de potência MBI vem sendo muito utilizado para investigação de técnicas de controle para regulação da tensão e manutenção de estabilidade transitória. Este sistema exibe aspectos qualitativos importantes do comportamento de um sistema multimáquinas e é relativamente simples e extremamente apropriado para a descrição de conceitos gerais de estabilidade de SEP's (Guo et al., 2001; Zhu et al., 1997; Wang e Hill, 1996; Wang et al., 1993; Wang et al., 1993).

Suponha que diferentes cargas acopladas ao barramento de cargas podem estar ativas ou não durante um período de operação do sistema de potência MBI. As variações neste barramento afetam os modos (condições) de operação do sistema. Nestas condições, a freqüência de ocorrência de cada carga no barramento pode ser determinada e, então, os modos de operação do sistema podem ser divididos de acordo com a carga que este supre. A Figura 5.3 ilustra a configuração adotada para o barramento de cargas do sistema. Esta descrição do barramento de cargas é bastante adequada para representação, por exemplo, de plantas de potência industriais em esquema de co-geração, onde a potência excedente pode ser enviada ao sistema global. No caso do sistema de potência MBI, supõe-se que o gerador fornece para a rede de transmissão uma potência cons- 


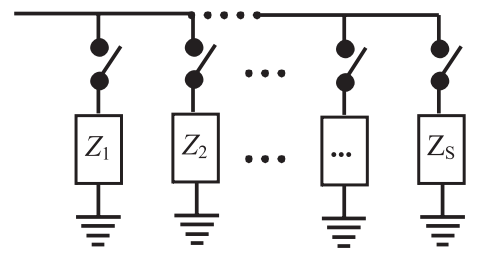

Figura 5.3: Configuração do barramento de cargas do sistema.

tante em cada modo de operação, ou seja, em cada um destes modos, a potência que não for utilizada na carga interna, será entregue ao sistema global, e isso é realizado considerando um modo de operação pré-determinado.

Agora, as variações abruptas e aleatórias de modos de operação são incluídas nas dinâmicas do sistema de potência MBI em esquema de co-geração (5.1) - (5.4). Para isto, considera-se que o sistema supre duas cargas diferentes: $Z_{1}=R_{1}+j X_{1}$ e $Z_{2}=R_{2}+j X_{2}$, com $R_{1}$ e $R_{2}$ as resistências [p.u.] e $X_{1}$ e $X_{2}$ as reatâncias [p.u.] Desta forma, pode-se determinar as correntes $I_{q}$ e $I_{d}$ como

$$
\begin{aligned}
& I_{q}=K_{1 q}(r) \cos \delta+K_{2 q}(r) \sin \delta+K_{3 q}(r) E_{q}^{\prime} \\
& I_{d}=K_{1 d}(r) \cos \delta+K_{2 d}(r) \sin \delta+K_{3 d}(r) E_{q}^{\prime}
\end{aligned}
$$

onde

$$
\begin{aligned}
& K_{1 q}(r):=-\frac{\left[a_{2}\left(1+k_{1}\right)-a_{1} k_{2}\right] V_{\infty}}{a_{1}^{2}+a_{2}^{2}} \\
& K_{2 q}(r):=\frac{\left[a_{1}\left(1+k_{1}\right)+a_{2} k_{2}\right] V_{\infty}}{a_{1}^{2}+a_{2}^{2}} \\
& K_{3 q}(r):=\frac{a_{2}\left[\left(1+k_{1}\right)^{2}+k_{2}^{2}\right]}{a_{1}^{2}+a_{2}^{2}} \\
& K_{1 d}(r):=K_{2 q} \\
& K_{2 d}(r):=-K_{1 q} \\
& K_{3 d}(r):=-\frac{a_{1}\left[\left(1+k_{1}\right)^{2}+k_{2}^{2}\right]}{a_{1}^{2}+a_{2}^{2}}
\end{aligned}
$$

são parâmetros que variam de acordo com a carga equivalente do barramento de cargas do sistema, com $k_{1}=\frac{X_{e q} x_{e}}{R_{e q}^{2}+X_{e q}^{2}}, k_{2}=\frac{R_{e q} x_{e}}{R_{e q}^{2}+X_{e q}^{2}}, a_{1}=\left[\left(1+k_{1}\right)^{2}+k_{2}^{2}\right] x_{d}^{\prime}+\left(1+k_{1}\right) x_{e} \mathrm{e}$ $a_{2}=k_{2} x_{e}$ e $V_{\infty}$ é a tensão no barramento-infinito [p.u.]. A dinâmica das condições de 


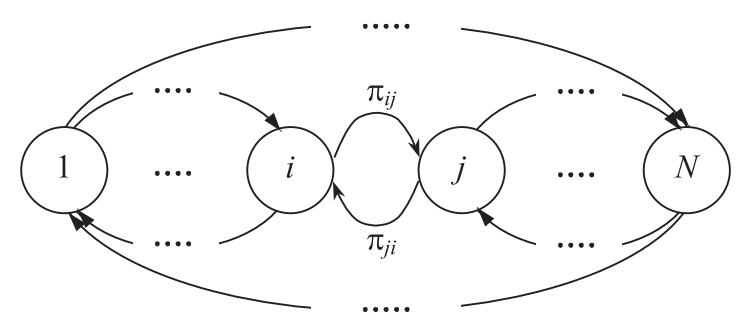

Figura 5.4: Modos de transição do sistema de potência com variações abruptas e aleatórias de cargas.

operação do sistema de potência de acordo com as variações de carga é modelada como uma cadeia de Markov com três diferentes modos $(N=3)$, correspondendo às possíveis combinações entre as cargas $Z_{1}$ e $Z_{2}$ :

- modo 1 - somente carga $Z_{1}, Z_{e q}=Z_{1}$;

- modo 2 - somente carga $Z_{2}, Z_{e q}=Z_{2}$;

- modo 3 - ambas cargas $Z_{1}$ e $Z_{2}, Z_{e q}=\frac{Z_{1} Z_{2}}{Z 1+Z_{2}}$.

Desta forma, o conjunto dos modos de operação do sistema de potência MBI em esquema de co-geração é dado por $\mathbb{S}=\{1,2,3\}$. Agora, suponha que as variações de carga são governadas pelo processo estocástico $r$, que é o processo de Markov de modos-finitos assumindo valores no conjunto $\mathbb{S}$, cujas transições podem ser definidas pela monitoração dos instantes de ativação e desativação de cargas. A matriz de taxas de transição $\Pi=\left[\pi_{i j}\right]_{i, j \in \mathbb{S}}$, que descreve a evolução no tempo do processo de Markov $r$, pode ser determinada de um banco de dados (histórico) da operação do sistema, logo, quanto maior o banco de dados, mais precisa é a definição de П. A Figura 5.4 ilustra a transição entre os modos de operação do sistema de acordo com as variações de carga.

Considerando um histórico da operação do sistema em que a distribuição estacionária do processo é dada por $\mu=(0.30,0.20,0.50)$, ou seja, a percentagem de operação em cada modo do sistema corresponde a $30 \%$ no modo 1, $20 \%$ no modo 2 e $50 \%$ no modo 3 , pode-se determinar os elementos da matriz $\Pi$ usando a seguinte relação (Papoulis, 1984)

$$
\pi_{i i} \mu_{i}=\sum_{j=1 ; j \neq i}^{3} \pi_{i j} \mu_{j}
$$

Através deste mesmo histórico, também pode-se definir o modo de operação nominal do 
sistema como sendo o modo 3 , visto que, é o modo no qual a percentagem de operação é maior.

Então, usando (5.10) e (5.11), as dinâmicas do sistema de potência MBI com variações abruptas e aleatórias de condições de operação são dadas por

$$
\begin{aligned}
\dot{\delta} & =\omega_{0} \omega \\
\dot{\omega} & =\frac{1}{2 H}\left[P_{m}-E_{q}^{\prime}\left(K_{1 q}(r) \cos \delta+K_{2 q}(r) \sin \delta+K_{3 q}(r) E_{q}^{\prime}\right)\right] \\
\dot{E}_{q}^{\prime} & =\frac{1}{\tau_{d o}^{\prime}}\left[E_{f d}-E_{q}^{\prime}+\left(x_{d}-x_{d}^{\prime}\right)\left(K_{1 d}(r) \cos \delta+K_{2 d}(r) \sin \delta+K_{3 d}(r) E_{q}^{\prime}\right)\right](5 . \\
\dot{E}_{f d} & =\frac{1}{T_{e}}\left[K_{e}\left(V_{r e f}(r)-\left|V_{t}(r)\right|+V_{s}\right)-E_{f d}\right]
\end{aligned}
$$

Adotando $x=\left[\delta, \omega, E_{q}^{\prime}, E_{f d}\right]^{T}$ como o vetor das variáveis de estado e $u=V_{s}$ como o vetor de entrada, o sistema de potência MBI pode ser descrito na forma espaço de estado

$$
\dot{x}=f(x, r)+B u
$$

onde

$$
\begin{aligned}
f(x, r):= & {\left[\begin{array}{l}
\omega_{0} x_{2} \\
\frac{1}{2 H}\left[P_{m}-x_{3}\left(K_{1 q}(r) \cos \left(x_{1}\right)+K_{2 q}(r) \sin \left(x_{1}\right)+K_{3 q}(r) x_{3}\right)\right] \\
\frac{1}{\tau_{d o}^{\prime}}\left[x_{4}-x_{3}+\left(x_{d}-x_{d}^{\prime}\right)\left(K_{1 d}(r) \cos \left(x_{1}\right)+K_{2 d}(r) \sin \left(x_{1}\right)+K_{3 d}(r) x_{3}\right)\right] \\
\frac{1}{T_{e}}\left[K_{e}\left(V_{r e f}(r)-\left|V_{t}(x, r)\right|\right)-x_{4}\right]
\end{array}\right] } \\
B:= & {\left[\begin{array}{c}
0 \\
0 \\
0 \\
\frac{K_{e}}{T_{e}}
\end{array}\right] ; } \\
\left|V_{t}(x, r)\right|= & \left\{\left[x_{3}+x_{d}^{\prime}\left(K_{1 d}(r) \cos \left(x_{1}\right)+K_{2 d}(r) \sin \left(x_{1}\right)+K_{3 d}(r) x_{3}\right)\right]^{2}\right. \\
& \left.+\left[x_{d}^{\prime}\left(K_{1 q}(r) \cos \left(x_{1}\right)+K_{2 q}(r) \sin \left(x_{1}\right)+K_{3 q}(r) x_{3}\right)\right]^{2}\right\}^{1 / 2} .
\end{aligned}
$$




\subsubsection{Relações de equilíbrio}

O equilíbrio do sistema de potência MBI (5.23) pode ser obtido quando $\dot{x}=0$ em $x$ e em $u$, o que resulta em

$$
\begin{aligned}
x_{1 e} & =\text { especificado } \\
x_{2 e} & =0 \\
x_{3 e} & =\frac{-\left[K_{1 q} \cos \left(x_{1 e}\right)+K_{2 q} \sin \left(x_{1}\right)\right]+\left[\left(K_{1 q} \cos \left(x_{1 e}\right)+K_{2 q} \sin \left(x_{1 e}\right)\right)^{2}+4 P_{m} K_{3 q}\right]^{1 / 2}}{2 K_{3 q}} \\
x_{4 e} & =\left(x_{d}^{\prime}-x_{d}\right)\left[K_{1 d} \cos \left(x_{1 e}\right)+K_{2 d} \sin \left(x_{1 e}\right)\right]+\left[1+\left(x_{d}^{\prime}-x_{d}\right) K_{3 d}\right] x_{3 e} \\
u_{e} & =0 \\
V_{r e f_{e}} & =\frac{1}{K_{e}} x_{4 e}+\left|V_{t}\right|
\end{aligned}
$$

onde os parâmetros $K_{1 q}, K_{2 q}, K_{3 q}, K_{1 d}, K_{2 d}, K_{3 d}$ e $V_{r e f_{e}}$ devem ser calculados para cada modo de operação. O ângulo $x_{1 e}$ deve ser especificado de acordo com a potência a ser liberada pelo sistema de potência. Neste exemplo, considere que, em condições de operação nominais (modo 3 ), o ângulo de potência é mantido na faixa $x_{1} \cong 0.7328 \pm 10 \%$ rad que assegura uma potência ativa na faixa $P=1.0 \pm 10 \%$ p.u. e a potência reativa de acordo com as condições de operação desejadas (por exemplo, para o sistema sem cargas $Q=0.5)$. Valores de $x_{1}$ maiores que a faixa considerada ocasionarão perda de estabilidade transitória do sistema de potência, visto que, a potência ativa $P$ entregue pelo gerador, neste exemplo, é considerada constante e igual a potência mecânica $P_{m}$ na entrada do sistema. Detalhes sobre o estudo de estabilidade transitória em sistemas de potência podem ser obtidos em Bretas e Alberto (1999).

\subsection{Modelagem e Controle Fuzzy do SEP em Esquema de Co-Geração}

Nesta seção serão apresentados os resultados obtidos com a aplicação da abordagem fuzzy proposta na estabilização do sistema MBI em esquema de co-geração. Detalha-se inicialmente o procedimento para obtenção da representação fuzzy do sistema. Posteriormente são apresentados os critérios utilizados nos diferentes projetos de controladores fuzzy propostos nos Capítulos 3 e 4, bem como os resultados de simulações. Com o uso 


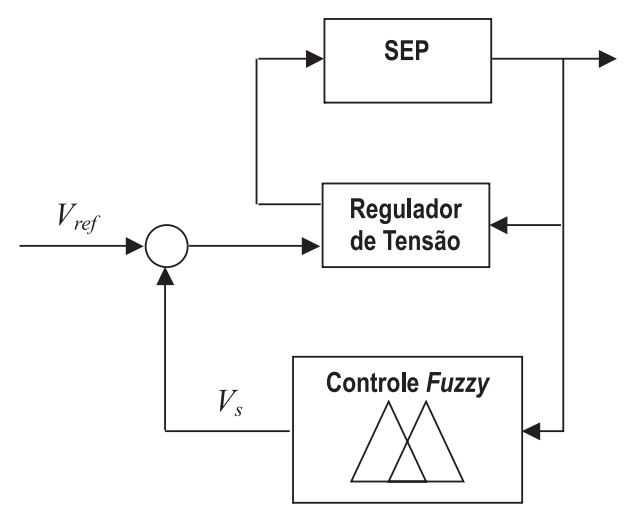

Figura 5.5: Diagrama em blocos do sistema de controle do SEP.

da modelagem fuzzy desenvolvida, representa-se as dinâmicas do sistema de potência MBI em esquema de co-geração, visando a obtenção de um controlador fuzzy coordenado com um regulador automático de tensão. O objetivo do sistema de controle coordenado é manter a estabilidade do sistema durante a ocorrência das variações nas cargas, além de reduzir as oscilações eletromecânicas, para garantir a eficiência da operação. A Figura 5.5 apresenta o diagrama em blocos do sistema de controle usando o controlador fuzzy proposto. Resultados preliminares de estabilização de um sistema de potência MBI com variações na tensão de barramento-infinito, obtidos usando a abordagem fuzzy proposta podem ser encontradas em Arrifano e Oliveira (n.d.b). Resultados de estabilização para o sistema de potência MBI em esquema de co-geração, obtidos usando cargas puramente reativas, ou seja, cargas do tipo $Z_{1}=j X_{1}$ e $Z_{2}=j X_{2}$, podem ser encontrados em Arrifano et al. (2004). Na prática, por questões de projeto, considera-se que a tensão do barramento-infinito $V_{\infty}$ deve ser constante, daí a mudança na formulação do exemplo de projeto, agora descrevendo as variações do barramento de cargas do sistema de potência MBI.

\subsubsection{Modelagem fuzzy}

Tendo em vista o projeto de controle fuzzy, dado que o equilíbrio do sistema $x_{e} \neq 0$, é necessário fazer uma mudança de coordenadas no sistema (5.23), que desloque o seu equilíbrio para a origem (veja Observação 10). Desta forma, seja $\xi:=x-x_{e}$ o estado deslocado do sistema, então o sistema de potência MBI (5.23) pode ser reescrito na 
seguinte forma

$$
\dot{\xi}=f\left(\xi+x_{e}, r\right)+B u
$$

onde $B$ é uma matriz constante,

$$
\begin{aligned}
& f\left(\xi+x_{e}, r\right):=\left[\begin{array}{ll}
\omega_{0} & \left(\xi_{2}+x_{2 e}\right) \\
\frac{1}{2 H} & {\left[P_{m}-\left(\xi_{3}+x_{3 e}\right)\left(K_{1 q}(r) \cos \left(\xi_{1}+x_{1 e}\right)\right.\right.} \\
& \left.+K_{2 q}(r) \sin \left(\xi_{1}+x_{1 e}\right)+K_{3 q}(r)\left(\xi_{3}+x_{3 e}\right)\right] \\
\frac{1}{\tau_{d o}^{\prime}} & {\left[\left(\xi_{4}+x_{4 e}\right)-\left(\xi_{3}+x_{3 e}\right)+\left(x d-x_{d}^{\prime}\right)\left(K_{1 d}(r) \cos \left(\xi_{1}+x_{1 e}\right)\right.\right.} \\
& \left.\left.+K_{2 d}(r) \sin \left(\xi_{1}+x_{1 e}\right)+K_{3 d}(r)\left(\xi_{3}+x_{3 e}\right)\right)\right] \\
\frac{1}{T_{e}} & {\left[K_{e}\left(V_{r e f}(r)-\left|V_{t}\left(\xi+x_{e}, r\right)\right|\right)-\left(\xi_{4}+x_{4 e}\right)\right]}
\end{array}\right] \\
& \left|V_{t}\left(\xi+x_{e}, r\right)\right|:=\left\{\left[\left(\xi_{3}+x_{3 e}\right)+x_{d}^{\prime}\left(K_{1 d}(r) \cos \left(\xi_{1}+x_{1 e}\right)+K_{2 d}(r) \sin \left(\xi_{1}+x_{1 e}\right)\right.\right.\right. \\
& \left.\left.+K_{3 d}(r)\left(\xi_{3}+x_{3 e}\right)\right)\right]^{2}+\left[x _ { d } ^ { \prime } \left(K_{1 q}(r) \cos \left(\xi_{1}+x_{1 e}\right)+K_{2 q}(r) \sin \left(\xi_{1}+x_{1 e}\right)\right.\right. \\
& \left.\left.\left.+K_{3 q}(r)\left(\xi_{3}+x_{3 e}\right)\right)\right]^{2}\right\}^{1 / 2} \text {. }
\end{aligned}
$$

Os valores numéricos dos parâmetros físicos adotados são: $\omega_{0}=377, H=5, P_{m}=1$, $\tau_{d o}^{\prime}=6, T_{e}=0.01, K_{e}=100, V_{\infty}=0.89 \angle 0, x_{d}^{\prime}=0.32, x_{d}=1.60, x_{e}=0.40, r_{1}=-4.5$, $r_{2}=-3.0, x_{1}=6.5$ e $x_{2}=4.3$. A Tabela 5.1 contém os valores dos parâmetros $K_{1 q}$, $K_{2 q}, K_{3 q}, K_{1 d}, K_{2 d}, K_{3 d}$ e $V_{\text {ref }}$ calculados para cada modo de operação.

Tabela 5.1: Parâmetros do sistema de potência para diferentes condições de operação.

\begin{tabular}{c|c|c|c|c|c|c|c}
\hline Modo & $K_{1 q}$ & $K_{2 q}$ & $K_{3 q}$ & $K_{1 d}$ & $K_{2 d}$ & $K_{3 d}$ & $V_{\text {ref }}$ \\
\hline $\mathbf{1}$ & -0.0153 & 1.2195 & -0.0214 & 1.2195 & 0.0153 & -1.4207 & 1.0505 \\
\hline $\mathbf{2}$ & -0.0226 & 1.2083 & -0.0315 & 1.2083 & 0.0226 & -1.4363 & 1.0674 \\
\hline $\mathbf{3}$ & -0.0363 & 1.1863 & -0.0507 & 1.1863 & 0.0363 & -1.4671 & 1.1079 \\
\hline
\end{tabular}

Para $x_{1} \approx 0.7328 \pm 10 \%$, define-se que a representação fuzzy será construída com duas representações lineares locais $(R=2)$ em cada modo em torno dos seguintes pontos de linearização $\bar{x}$

- modo 1

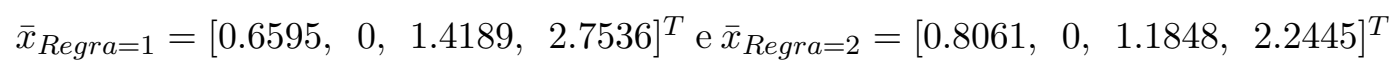

- modo 2 


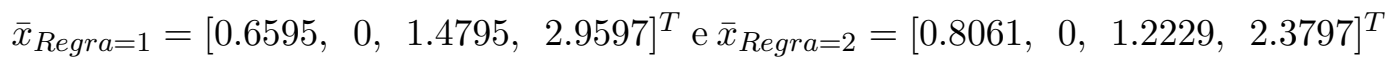

- modo 3

$\bar{x}_{\text {Regra }=1}=\left[\begin{array}{llll}0.6595, & 0, & 1.6237, & 3.4445\end{array}\right]^{T}$ e $\bar{x}_{\text {Regra }=2}=\left[\begin{array}{llll}0.8061, & 0, & 1.3079, & 2.6794\end{array}\right]^{T}$.

Deste forma, pode-se obter as matrizes $A_{i j}, i=1,2,3, j=1,2$ a partir da função $f(\cdot, \cdot)$ do sistema de potência usando a fórmula de linearização de Teixeira \& Żak apresentada na Seção 2.2.2. Esta fórmula pode ser implementada no Matlab usando basicamente os comandos syms, para declaração das variáveis simbólicas, e jacobian( ), para calcular o gradiente de $f(\cdot, \cdot)$, ambos disponíveis no módulo Symbolic Math. As matrizes resultantes são

$$
\begin{aligned}
& \text { - modo } 1 \\
& A_{11}=\left[\begin{array}{rrrr}
0 & 377 & 0 & 0 \\
-0.1378 & 0 & -0.0679 & -0.0011 \\
-0.1569 & 0 & -0.4695 & 0.1672 \\
1670 & 0 & -5326.3 & -101.08
\end{array}\right] ; B_{11}=B=\left[\begin{array}{r}
0 \\
0 \\
0 \\
10^{4}
\end{array}\right] \text {; } \\
& A_{12}=\left[\begin{array}{rrrr}
0 & 377 & 0 & 0 \\
-0.1016 & 0 & -0.0814 & 0.0008 \\
-0.1853 & 0 & -0.4699 & 0.1662 \\
1898.1 & 0 & -5213.4 & -99.38
\end{array}\right] ; B_{12}=B
\end{aligned}
$$

- modo 2

$$
\begin{aligned}
& A_{21}=\left[\begin{array}{rrrr}
0 & 377 & 0 & 0 \\
-0.1431 & 0 & -0.0634 & -0.0012 \\
-0.1542 & 0 & -0.4729 & 0.1672 \\
1676.5 & 0 & -5285 & -100.87
\end{array}\right] ; B_{21}=B ; \\
& 0 \\
& A_{22}=\left[\begin{array}{rrrr}
377 & 0 & 0 \\
-0.1046 & 0 & -0.0775 & 0.0009 \\
-0.1825 & 0 & -0.4733 & 0.1662 \\
1901.9 & 0 & -5175.9 & -99.57
\end{array}\right] ; B_{22}=B ;
\end{aligned}
$$

- modo 3

$$
A_{31}=\left[\begin{array}{rrrr}
0 & 377 & 0 & 0 \\
-0.1556 & 0 & -0.0540 & -0.0016 \\
-0.1490 & 0 & -0.4795 & 0.1672 \\
1692.4 & 0 & -5204.4 & -100.26
\end{array}\right] ; B_{31}=B
$$




$$
A_{32}=\left[\begin{array}{rrrr}
0 & 377 & 0 & 0 \\
-0.1111 & 0 & -0.0693 & 0.0011 \\
-0.1771 & 0 & -0.4798 & 0.1662 \\
1910.9 & 0 & -5102.6 & -100.10
\end{array}\right] ; B_{32}=B
$$

As funções características $m_{i}(\cdot), i=1,2,3$ são funções definidas no conjunto $\{0,1\}$, representando os modos de operação do sistema de potência MBI em esquema de cogeração. Neste exemplo, dado que a variável premissa $z$ é determinada pela monitoração dos instantes de ativação das cargas, $m_{i}(z)=1$, quando $z \in M_{i}$ e $m_{i}(z)=0$, caso contrário. As funções de pertinência normalizadas $n_{i j}(\cdot), j=1,2$ descrevem faixas das variáveis de estado $x_{1}$ e $x_{3}$ em cada modo, como mostrado na Figura 5.6 e são obtidas usando o comando gaussmf ( ) disponível no módulo Fuzzy Logic do Matlab, o qual gera uma função de pertinência gaussiana. Neste exemplo, a faixa de interesse é determinada pela variação de $x_{1}$ no intervalo [0.6595, 0.8061].

O SFSM usado para representar as dinâmicas do sistema MBI (5.24) é dado por

Modo 1: $\quad$ SE $z \in M_{1}$, ENTÃO

Regra 1: $\quad$ SE $\xi_{1}$ é $N_{111}$ e $\xi_{3}$ é $N_{113}$

$$
\text { ENTÃO } \dot{\xi}=A_{11} \xi+B_{11} u
$$

Regra 2: $\quad$ SE $\xi_{1}$ é $N_{121}$ e $\xi_{3}$ é $N_{123}$

$$
\text { ENTÃO } \dot{\xi}=A_{12} \xi+B_{12} u
$$

Modo 2: SE $z \in M_{2}$, ENTÃO

$$
\begin{array}{ll}
\text { Regra 1: } & \text { SE } \xi_{1} \text { é } N_{211} \text { e } \xi_{3} \text { é } N_{213} \\
& \text { ENTÃO } \dot{\xi}=A_{21} \xi+B_{21} u \\
\text { Regra 2: } & \text { SE } \xi_{1} \text { é } N_{221} \text { e } \xi_{3} \text { é } N_{223} \\
& \text { ENTÃO } \dot{\xi}=A_{22} \xi+B_{22} u
\end{array}
$$



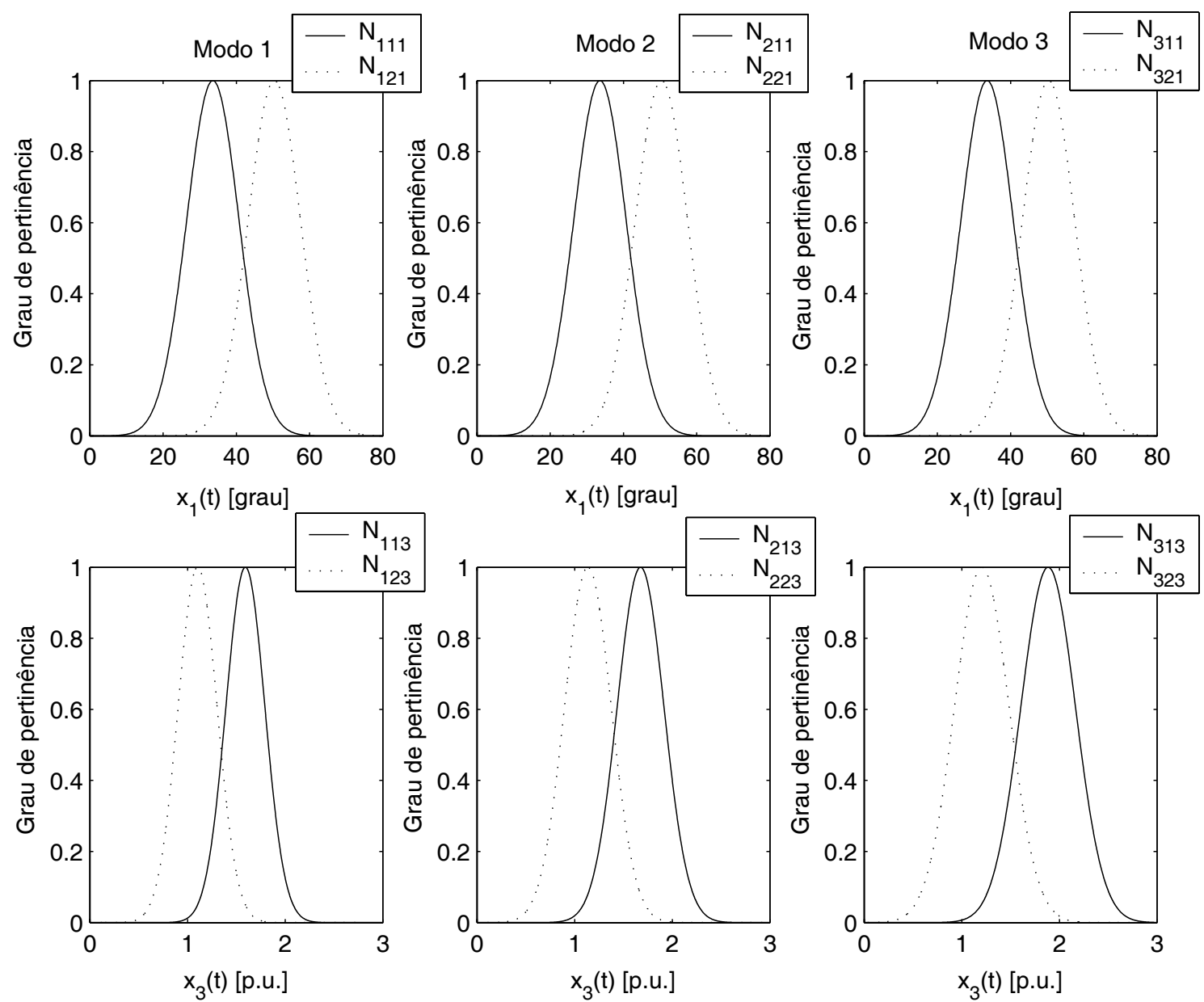

Figura 5.6: Funções de pertinência adotadas.

Modo 3: $\quad$ SE $z \in M_{3}$, ENTÃO

Regra 1: $\quad$ SE $\xi_{1}$ é $N_{311}$ e $\xi_{3}$ é $N_{313}$

$$
\text { ENTÃO } \dot{\xi}=A_{31} \xi+B_{31} u
$$

Regra 2: $\quad$ SE $\xi_{1}$ é $N_{321}$ e $\xi_{3}$ é $N_{323}$

ENTÃO $\dot{\xi}=A_{32} \xi+B_{32} u$

e o SFSM resultante é descrito por

$$
\dot{\xi}=\sum_{i=1}^{3} \sum_{j=1}^{2} m_{i}(z) n_{i j}(\xi)\left(A_{i j} \xi+B_{i j} u\right) .
$$




\subsubsection{Projeto de controle fuzzy estabilizante}

A lei de controle fuzzy apresenta a mesma estrutura do SFSM (5.25), i.e.,

$$
u=-\sum_{i=1}^{3} \sum_{j=1}^{2} m_{i}(z) n_{i j}(\xi) K_{i j} \xi
$$

onde o conhecimento do sistema é repassado através das funções das variáveis premissas $z$ e $\xi$. O objetivo agora é a determinação dos ganhos de realimentação de estado $K_{i j}$, $i=1,2,3, j=1,2$, de forma a garantir as seguintes especificações:

- manter o sistema operando no modo de operação nominal (modo $3-x_{1}=0.7328$ $\operatorname{rad}\left(\approx 42^{\circ}\right), P=1.0$ p.u. e $Q=1.12$ p.u. $)$;

- diminuir as oscilações eletromecânicas na dinâmica do sistema;

- manter a tensão terminal do gerador permitindo variações na faixa de até $10 \%$ do valor nominal ( $V_{t}=1$ p.u.).

Uma característica das representações lineares locais do sistema de potência MBI, matrizes $A_{i j}, i=1,2,3, j=1,2$, é a existência de pólos complexos instáveis, como mostrado na Tabela 5.2. Como antes mencionado (veja Observação 6), a estabilidade de cada modo pode ser preservada especificando $\pi_{i}>2 \operatorname{Re}\left\{\lambda_{\max _{j}}\left[A_{i j}\right]\right\}$. Então, dado que $\pi_{i}=-\pi_{i i}=\sum_{j=1, j \neq i}^{N} \pi_{i j}$, escolhendo $\pi_{1}=1.08, \pi_{2}=1.08, \pi_{3}=1.10$ e usando (5.18), pode-se obter a seguinte matriz de taxas de transição

$$
\Pi=\left[\begin{array}{rrr}
-1.08 & 0.27 & 0.81 \\
0.68 & -1.08 & 0.40 \\
0.24 & 0.86 & -1.10
\end{array}\right]
$$

Adota-se o como vetor de estado inicial $x(0)=\left[\begin{array}{llll}0.7328, & -0.001, & 1.4420, & 2.9903\end{array}\right]^{T}$ e como modo inicial $r(0)=3$. Todos os projetos de controladores fuzzy apresentados nos Capítulos 3 e 4 utilizam as matrizes $\left(A_{i j}, B_{i j}, \Pi\right), i=1,2,3, j=1,2$. No caso do projeto de controle fuzzy estabilizante com índice de desempenho, ainda são necessários os seguintes parâmetros de projeto:

- taxas de decaimento 
Tabela 5.2: Pólos do sistema de potência MBI em malha-aberta.

\begin{tabular}{c|cccc}
\hline Modo & \multicolumn{4}{|c}{ Vetores de Pólos } \\
\hline \multirow{2}{*}{$\mathbf{1}$} & $\lambda_{11}=\left[\begin{array}{llll}-91.36 & 0.0523+j 7.7251 & 0.0523-j 7.7251 & -10.30\end{array}\right]^{T}$ \\
& $\lambda_{12}=\left[\begin{array}{llll}-89.57 & -11.35 & 0.5355+j 6.5217 & 0.5355-j 6.5217\end{array}\right]^{T}$ \\
\hline \multirow{2}{*}{$\mathbf{2}$} & $\lambda_{21}=\left[\begin{array}{llll}-91.22 & 0.0157+j 7.8645 & 0.0157-j 7.8645 & -10.16\end{array}\right]^{T}$ \\
& $\lambda_{22}=\left[\begin{array}{llll}-89.85 & 0.5379+j 6.5596 & 0.5379-j 6.5596 & -11.27\end{array}\right]^{T}$ \\
\hline \multirow{2}{*}{$\mathbf{3}$} & $\lambda_{31}=\left[\begin{array}{llll}-90.73 & -0.0765+j 8.2092 & -0.0765-j 8.2092 & -9.85\end{array}\right]^{T}$ \\
& $\lambda_{32}=\left[\begin{array}{llll}-90.57 & -11.10 & 0.5490+j 6.6391 & 0.5490-j 6.6391\end{array}\right]^{T}$ \\
\hline
\end{tabular}

$$
\alpha_{1}=\alpha_{2}=\alpha_{3}=0.1
$$

- restrições na entrada de controle

$$
\mu_{1}=\mu_{2}=\mu_{3}=2 \text {. }
$$

No caso do projeto de controle fuzzy $\mathcal{H}_{\infty}$ robusto, que utiliza o erro de aproximação $\Delta f$ entre o sistema MBI (5.24) e o sistema fuzzy (5.25), dado que $\Delta g=0$, são necessárias as matrizes limitantes para os erros de aproximação e as matrizes de ponderação escolhidas para cada modo, respectivamente, que são

$$
A_{\mathbf{p} 1}=\left[\begin{array}{rrrr}
0 & 0 & 0 & 0 \\
0.0001 & 0 & 0.0001 & 0 \\
0.0002 & 0 & 0 & 0 \\
0.2782 & 0 & 5.5409 & 0.1158
\end{array}\right] ; S_{1}=\operatorname{diag}\left\{\begin{array}{llll}
20 & 20 & 0.009 & 0.009\}
\end{array}\right.
$$

- modo 2

$$
A_{\mathbf{p} 2}=\left[\begin{array}{rrrr}
0 & 0 & 0 & 0 \\
0.0001 & 0 & 0.0001 & 0 \\
0.0002 & 0 & 0 & 0 \\
0.3051 & 0 & 4.6306 & 0.0972
\end{array}\right] ; S_{2}=\operatorname{diag}\left\{\begin{array}{llll}
20 & 20 & 0.009 & 0.009\}
\end{array}\right.
$$

- modo 3

$$
A_{\mathbf{p} 3}=\left[\begin{array}{rrrr}
0 & 0 & 0 & 0 \\
0.0001 & 0 & 0.0001 & 0 \\
0.0002 & 0 & 0 & 0 \\
0.3086 & 0 & 3.1297 & 0.0663
\end{array}\right] ; S_{3}=\operatorname{diag}\left\{\begin{array}{llll}
20 & 20 & 0.009 & 0.009
\end{array}\right\} \text {. }
$$

O procedimento usado para obtenção do erro de aproximação $\Delta f$ é apresentado no Apêndice A. 


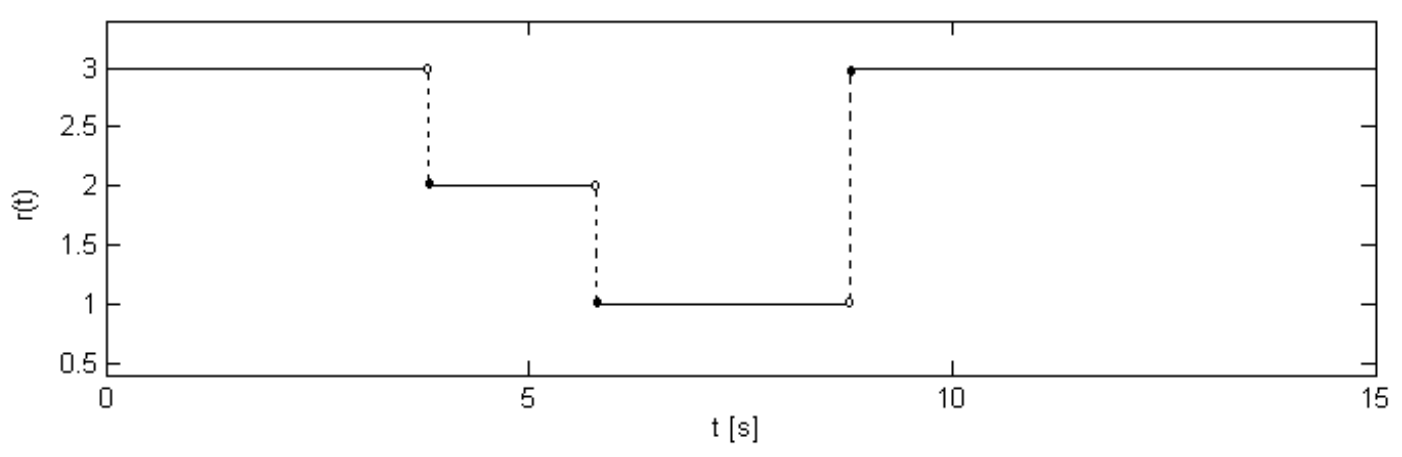

Figura 5.7: Mudanças de condições de operação durante a operação do sistema de potência MBI.

Com a distribuição $\mu$ e a matriz de taxas de transição $\Pi$, previamente definidas, utiliza-se o programa disponível em Waner e Costenoble (1997) para simular as transições entre os modos de operação do sistema de potência MBI descritas pelo processo de saltos Markovianos $r$. A Figura 5.7 mostra evolução no tempo de $r$.

Para testar a eficiência do controlador fuzzy $\mathcal{H}_{\infty}$ robusto, adiciona-se ao sistema de potência MBI (5.24) um distúrbio $w=\left[\begin{array}{lllll}0 & 10^{-2} \times \sin (2 \pi 20 t) & 0 & 0 & 0\end{array}\right]^{T}$, para simular uma perturbação na potência mecânica $P_{m}$ e, conseqüentemente, na velocidade do gerador $x_{2}$, originada de possíveis oscilações torcionais que podem ocorrer no conjunto turbinaeixo-rotor decorrentes das iterações existentes no acoplamento entre cada uma destas partes. A amplitude desta perturbação foi escolhida de forma a produzir uma variação de $\pm 10 \%$ em $P_{m}$ e a frequência $(20 \mathrm{~Hz})$ foi escolhida na faixa das oscilações que ocorrem na prática. Detalhes sobre a frequência das oscilações do conjunto turbina-eixo-rotor podem ser obtidos em Kundur (1994). Este tipo de perturbação é admissível para o sistema de potência MBI, visto que o conjunto turbina-eixo-rotor é considerado como um corpo rígido.

\subsection{Resultados de Simulação}

Os resultados de simulação do sistema de potência MBI em esquema de co-geração para os diferentes projetos de controle foram obtidos a partir de um conjunto de rotinas implementadas no Matlab, que são:

- obtenção das representações lineares locais; 
- projeto de controle fuzzy estabilizante;

- projeto de controle fuzzy estabilizante com índices de desempenho;

- projeto de controle fuzzy de custo garantido;

- projeto de controle fuzzy robusto;

- obtenção dos gráficos das respostas do sistema.

A obtenção das representações lineares locais, como antes mencionado, resume-se na obtenção das matrizes $A_{i j}$ e $B_{i j}, i=1,2,3, j=1,2$ utilizando a fórmula de linearização de Teixeira \& Żak. Estas matrizes, bem com a matriz $\Pi$ são os principais dados de entrada para os projetos de controle. As rotinas de projetos de controle fuzzy consistem na obtenção dos ganhos de realimentação de estado $K_{i j}, i=1,2,3, j=1,2$, solucionando um conjunto de LMI's dadas na Proposição 5 (controle fuzzy estabilizante), Problema 2 (controle fuzzy estabilizante com índice de desempenho), Problema 3 (controle fuzzy de custo garantido) e Problema 4 (controle fuzzy robusto) e foram implementadas usando os comandos setlmis([ ]), lmivar( ), lmiterm( ), getlmis, feasp( ) e $\operatorname{mincx}($ ), disponíveis no módulo LMI Control. Após o projeto de controle, são apresentados então os gráficos das respostas do sistema diante das variações no processo de Markov $r$, que foram implementados usando comandos básicos disponíveis no Matlab, cujos principais são os comandos ode45( ), para solução das equações diferenciais e plot( ), para visualização das curvas. Para facilitar a manipulação destas rotinas, implementou-se um menu de opções, via o comando menu( ), a partir do qual cada rotina é chamada dependendo da opção selecionada. A janela com o menu de opções é mostrada na Figura 5.8.

Para validação da abordagem fuzzy proposta, os resultados obtidos com o uso dos diferentes tipos de projetos de controle fuzzy na estabilização do sistema de potência MBI em esquema de co-geração são comparados com os resultados obtidos com o uso de um PSS clássico. O PSS clássico foi sintonizado usando a técnica de posicionamento de pólos de uma representação linear do sistema de potência MBI, obtida para as condições de operação nominal do sistema (modo 3) (Larsen e Swann, 1981). A estrutura básica deste PSS é mostrada na Figura 5.9 e os valores dos parâmetros utilizados na simulação são: $K_{P S S}=15, T_{W}=10, T_{1}=T_{3}=0.2148$ e $T_{2}=T_{4}=0.0595$. Além desta comparação, também são mostrados os resultados da aplicação do controle fuzzy no 


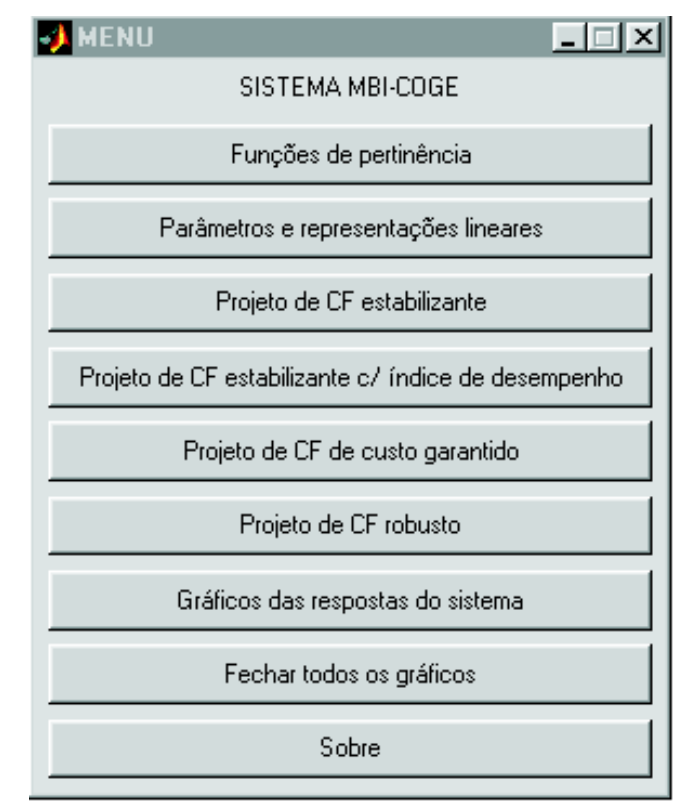

Figura 5.8: Menu de opções para simulação no Matlab.

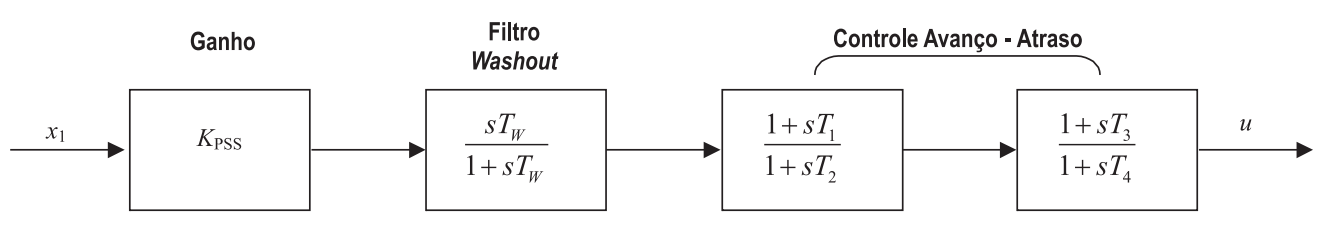

Figura 5.9: Diagrama em blocos do PSS.

sistema fuzzy (5.25), que foi construído para representar as dinâmicas do sistema de potência (5.24). Tendo em vista a eficiência dos resultados, estes são divididos em três casos:

- caso 1 - sistema de potência MBI com controle fuzzy estabilizante;

- caso 2 - sistema de potência MBI com controle fuzzy estabilizante com índice de desempenho;

- caso 3 - sistema de potência MBI com controle fuzzy robusto.

Os resultados dos projetos de controle para os Casos 1, 2 e 3 são mostrados nas Tabelas 5.3, 5.4 e 5.5, respectivamente. As Figuras 5.10 - 5.12, as Figuras $5.13-5.15$ e as Figuras 5.16 - 5.18 mostram as principais respostas do sistema para uma realização do experimento: variáveis de estado $x$, entrada de controle $u$ e tensão terminal $V_{t}$ e as 
dinâmicas das potências ativa $P$ e reativa $Q$ entregues pelo gerador ao sistema para os Casos 1, 2 e 3, respectivamente.

Os resultados obtidos para todos os casos são satisfatórios a medida que todas as especificações de projeto foram alcançadas e a modelagem fuzzy do sistema de potência MBI representa bem as dinâmicas não-lineares. Entretanto, vale ressaltar que, com as soluções de controle que utilizam critérios de desempenho (Casos 2 e 3), há uma redução ainda maior nas oscilações eletromecânicas do sistema, visto que, a velocidade de resposta do sistema em malha-fechada pode ser aumentada, especificando as taxas de decaimento $\alpha_{i}$ e matrizes de ponderação $S_{i}$ para os Caso 2 e 3 , respectivamente, em cada modo, tendo em vista que a abordagem fuzzy proposta é baseada em conceitos de estabilidade estocástica e, por isso, pode-se avaliar o desempenho do sistema em termos da velocidade de resposta, já que o sistema em malha-fechada é assintoticamente estável em torno de seu estado de equilíbrio no sentido da média-quadrática.

A vantagem de representar o sistema de potência MBI por um SFSM pode ser claramente observada: pode-se incluir uma descrição mais detalhada das mudanças do barramento de cargas, além de considerar as dinâmicas não-lineares do sistema no projeto. O uso destas informações aliado à condição de estabilidade estocástica produz condições menos conservadoras para a estabilidade usando controladores que produzem melhor desempenho no sistema. Além disso, usando técnicas de controle convencionais, como, por exemplo, o controle linear de posicionamento de pólos, o sistema em malhafechada deve ser estável em todos os modos de operação, enquanto que no sentido da estabilidade estocástica, a estabilidade para todos os modos não é uma condição necessária. No exemplo apresentado, os subsistemas que compõem o SFSM apresentam pólos complexos instáveis (veja Tabela 5.2) e a estabilidade de cada modo é assegurada através do cálculo da matriz $\Pi$, antes do projeto de controle. Sendo os resultados de estabilização garantidos no sentido da média-quadrática, ou seja, considera-se que a lei de controle fuzzy assintoticamente leva o sistema de uma condição inicial $\left(x_{0}, r_{0}\right)$ ao estado de equilíbrio no sentido da média-quadrática.

Nos resultados apresentados para ambos os Casos 1 e 2, o erro de aproximação descrito por $\Delta f$ é pequeno e pode ser ainda reduzido considerando uma faixa de variação menor da variável de estado $x_{1}$, ou um número maior de pontos de linearização, ou ainda, outros tipos de funções de pertinência. Um exemplo de projeto de controle fuzzy 
Tabela 5.3: Resultados do projeto de controle.

\begin{tabular}{|c|c|c|c|c|c|}
\hline \multicolumn{6}{|c|}{ Caso 1} \\
\hline Modo & & & Matrizes resulta & antes & \\
\hline \multirow{6}{*}{1} & $F_{11}=$ & -0.1697 & -0.22770 .5310 & $\begin{array}{ll}0 & 0.0099\end{array}$ & \\
\hline & $F_{12}=$ & -0.1932 & -0.18450 .518 & 8 0.0096] & \\
\hline & & 134.69 & -2299.5 & 115.91 & 15.987 \\
\hline & & -2299.5 & $1.5688 \times 10^{5}$ & -3029.6 & -340.37 \\
\hline & $P_{1}=$ & 115.91 & -3029.6 & 151.56 & 15.163 \\
\hline & & 15.987 & -340.37 & 15.163 & 2.4464 \\
\hline \multirow{6}{*}{2} & $F_{21}=$ & -0.1704 & $-0.2295 \quad 0.526$ & $\begin{array}{ll}9 & 0.0099]\end{array}$ & \\
\hline & $F_{22}=$ & -0.1935 & -0.19120 .515 & $2 \quad 0.0097]$ & \\
\hline & & 138.18 & -2319.4 & 115.2 & 16.220 \\
\hline & & -2319.4 & $1.5494 \times 10^{5}$ & -2935.3 & -339.20 \\
\hline & $P_{2}=$ & 115.2 & -2935.3 & 147.06 & 14.992 \\
\hline & & 16.220 & -339.20 & 14.992 & 2.4548 \\
\hline \multirow{6}{*}{3} & $F_{31}=$ & -0.1721 & $-0.2240 \quad 0.518$ & \begin{tabular}{|ll}
8 & 0.0098 \\
\end{tabular} & \\
\hline & $F_{32}=$ & -0.1942 & $-0.2019 \quad 0.508$ & 2 [ 0.0097$]$ & \\
\hline & & 143.31 & -2309.2 & 111.4 & 16.469 \\
\hline & & -2309.2 & $1.5377 \times 10^{5}$ & -2730.7 & -334.64 \\
\hline & $P_{3}=$ & 111.4 & -2730.7 & 137.74 & 14.494 \\
\hline & & 16.469 & -334.64 & 14.494 & 2.4797 \\
\hline
\end{tabular}

que considera a redução no erro de aproximação através da definição das funções de pertinência pode ser encontrado em Machado et al. (2003). É importante ressaltar que a utilização de um número maior de pontos de linearização leva a um aumento do número de regras que produz um aumento no número de condições LMI's a serem avaliadas, conseqüentemente, aumentando o conservadorismo da abordagem proposta.

Os resultados obtidos para o Caso 3 mostram a eficiência do desempenho do controle fuzzy robusto frente ao distúrbio e a variações abruptas e aleatórias de cargas. Como antes mencionado, o projeto de controle fuzzy $\mathcal{H}_{\infty}$ robusto apresentado no Capítulo 4 utiliza informações do erro de aproximação entre o SNLSM e o SFSM, o que garante o desempenho do controlador para o sistema não-linear em vez de apenas garantir o desempenho para o sistema fuzzy, como nos casos dos projetos de controladores fuzzy apresentados no Capítulo 3. A evolução no tempo do limitante para o erro de aproximação $\Delta f$ é apresentada na Figura 5.19. 
Tabela 5.4: Resultados do projeto de controle (cont.)

\begin{tabular}{|c|c|c|c|c|c|c|}
\hline \multicolumn{7}{|c|}{ Caso 2} \\
\hline Modo & \multicolumn{6}{|c|}{ Matrizes resultantes } \\
\hline \multirow{6}{*}{1} & \\
\hline & \multicolumn{6}{|c|}{$F_{12}=\left[\begin{array}{llll}-0.1792 & 3.8680 & 0.2356 & 0.0052\end{array}\right]$} \\
\hline & \multirow{4}{*}{$P_{1}=$} & & 0.0171 & $1 \quad 0.1810$ & -0.0072 & $-4.5118 \times 10^{-5}$ \\
\hline & & & 0.1810 & 111.52 & -3.8408 & -0.0601 \\
\hline & & & -0.0072 & -3.8408 & 0.2237 & 0.0036 \\
\hline & & -4 & $5118 \times 10^{-5}$ & -0.0601 & 0.0036 & $7.2777 \times 10^{-5}$ \\
\hline \multirow{6}{*}{2} & \multirow{2}{*}{\multicolumn{6}{|c|}{$\begin{array}{l}F_{21}=\left[\begin{array}{llll}-0.1567 & 5.3830 & 0.2097 & 0.0049\end{array}\right] \\
F_{22}=\left[\begin{array}{llll}-0.1802 & 4.0214 & 0.2237 & 0.0051\end{array}\right]\end{array}$}} \\
\hline & & {$[-0.1$} & & & $0051]$ & \\
\hline & \multirow{4}{*}{$P_{2}=$} & & 0.0173 & $3 \quad 0.1578$ & -0.0059 & $-2.1111 \times 10^{-5}$ \\
\hline & & & 0.1578 & 111.62 & -3.8058 & -0.0594 \\
\hline & & & -0.0059 & -3.8058 & 0.2184 & 0.0035 \\
\hline & & -2 & $1111 \times 10^{-5}$ & $5-0.0594$ & 0.0035 & $7.0936 \times 10^{-5}$ \\
\hline \multirow{6}{*}{3} & \multicolumn{6}{|c|}{$F_{31}=\left[\begin{array}{llll}-0.1734 & 2.0409 & 0.4305 & 0.0086\end{array}\right]$} \\
\hline & \multirow{5}{*}{\multicolumn{2}{|c|}{$P_{3}=$}} & -0.19490 . & $.5386 \quad 0.4530$ & $\begin{array}{ll}0 & 0.0091]\end{array}$ & \\
\hline & & & 0.0172 & -0.0246 & 0.0037 & 0.0002 \\
\hline & & & -0.0246 & 3.124 & 1.7469 & -0.0267 \\
\hline & & & 0.0037 & -1.7469 & 0.0720 & 0.0011 \\
\hline & & & 0.0002 & -0.0267 & 0.0011 & $3.2855 \times 10^{-5}$ \\
\hline
\end{tabular}

Tabela 5.5: Resultados do projeto de controle (cont.)

\begin{tabular}{c|ccrc}
\hline \multicolumn{5}{c}{ Caso 3 } \\
\hline Modo & Matrizes resultantes \\
\hline \multirow{4}{*}{$\mathbf{1}$} & $F_{11}=\left[\begin{array}{lrrr}0.1733 & -12.877 & 0.2703 & 0.0085\end{array}\right]$ \\
& $F_{12}=\left[\begin{array}{lrrr}0.2501 & -5.9279 & 0.2302 & 0.0085\end{array}\right]$ \\
& $P_{1}=\left[\begin{array}{rrrr}4.5790 & 100.14 & 0.2119 & 0.0002 \\
100.14 & 11393 & -54.692 & -0.0561 \\
0.2119 & -54.692 & 1.9375 & 0.0045 \\
0.0002 & -0.0561 & 0.0045 & 0.0001\end{array}\right]$ \\
\hline \multirow{3}{*}{$\mathbf{2}$} & $F_{21}=\left[\begin{array}{lrrr}0.1921 & -0.3563 & -0.2275 & -0.0016\end{array}\right]$ \\
& $F_{22}=\left[\begin{array}{lrrr}0.2040 & -0.1525 & -0.2240 & -0.0016\end{array}\right]$ \\
& $P_{2}=\left[\begin{array}{rrrr}4.5873 & 97.849 & 0.2549 & 0.0002 \\
97.849 & 11296 & -51.700 & -0.0574 \\
0.2549 & -51.70 & 1.8374 & 0.0039 \\
0.0002 & -0.0574 & 0.0039 & 0.0001\end{array}\right]$ \\
\hline \multirow{3}{*}{$\mathbf{3}$} & $F_{31}=\left[\begin{array}{lrrr}0.1887 & -14.886 & 0.0120 & 0.0045\end{array}\right]$ \\
& $F_{32}=\left[\begin{array}{lrrr}0.2966 & -3.8204 & -0.0401 & 0.0043\end{array}\right]$ \\
& $P_{3}=\left[\begin{array}{rrrr}4.5358 & 91.100 & 0.3298 & 0.0004 \\
91.100 & 10861 & -43.783 & -0.0056 \\
0.3298 & -43.783 & 1.5840 & 0.0028 \\
0.0004 & -0.0056 & 0.0028 & 0.0001\end{array}\right]$ \\
\hline \multicolumn{5}{|c}{ Ganho- $L_{2}$} \\
\hline
\end{tabular}



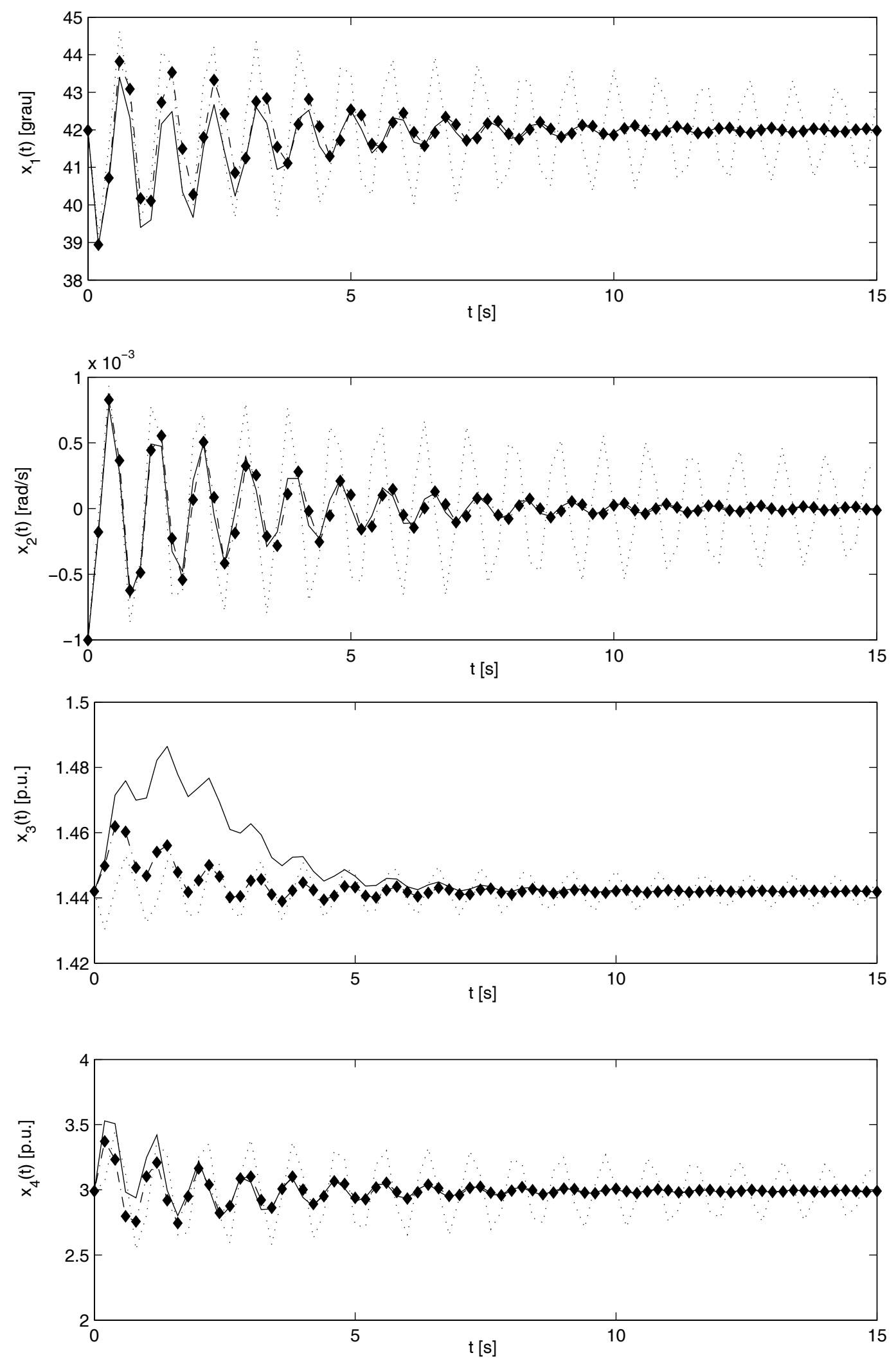

Figura 5.10: Caso 1 - Variáveis de estado: '-' sistema MBI com controle fuzzy, '...' sistema MBI com PSS clássico e '-॰-' SFSM com controle fuzzy. 


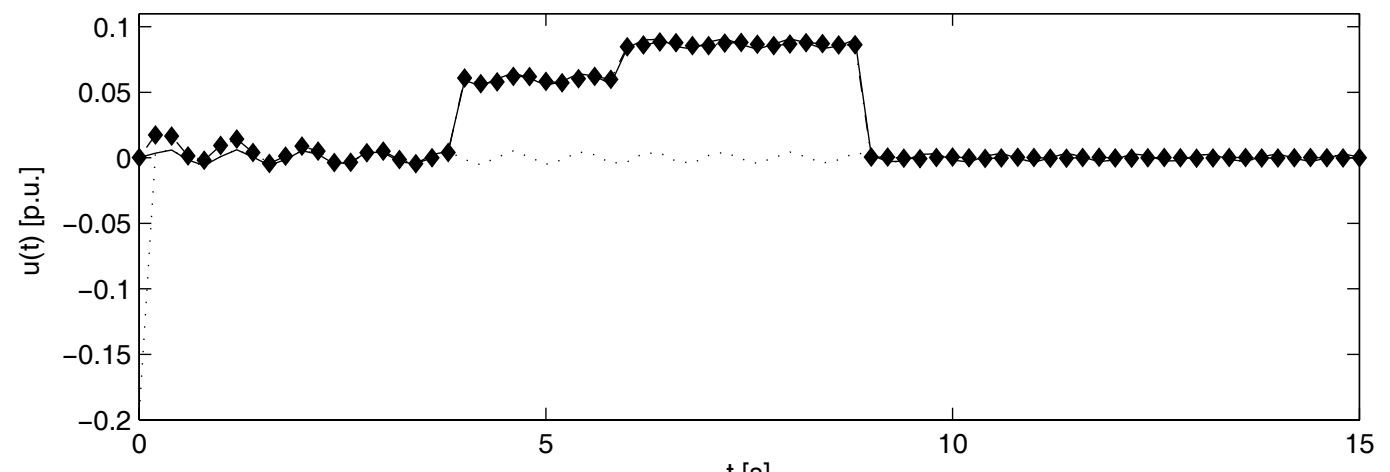

$\mathrm{t}[\mathrm{s}]$

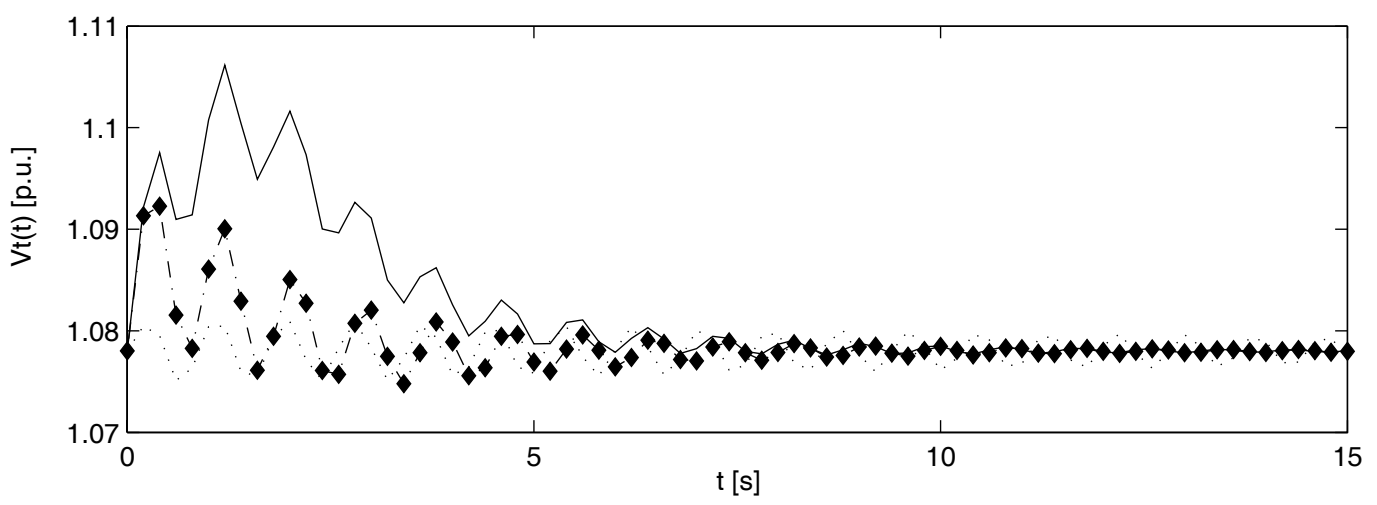

Figura 5.11: Caso 1 - Entrada de controle e tensão terminal: '-' sistema MBI com controle fuzzy, '...' sistema MBI com PSS clássico e '- $\diamond$ ' SFSM com controle fuzzy. 

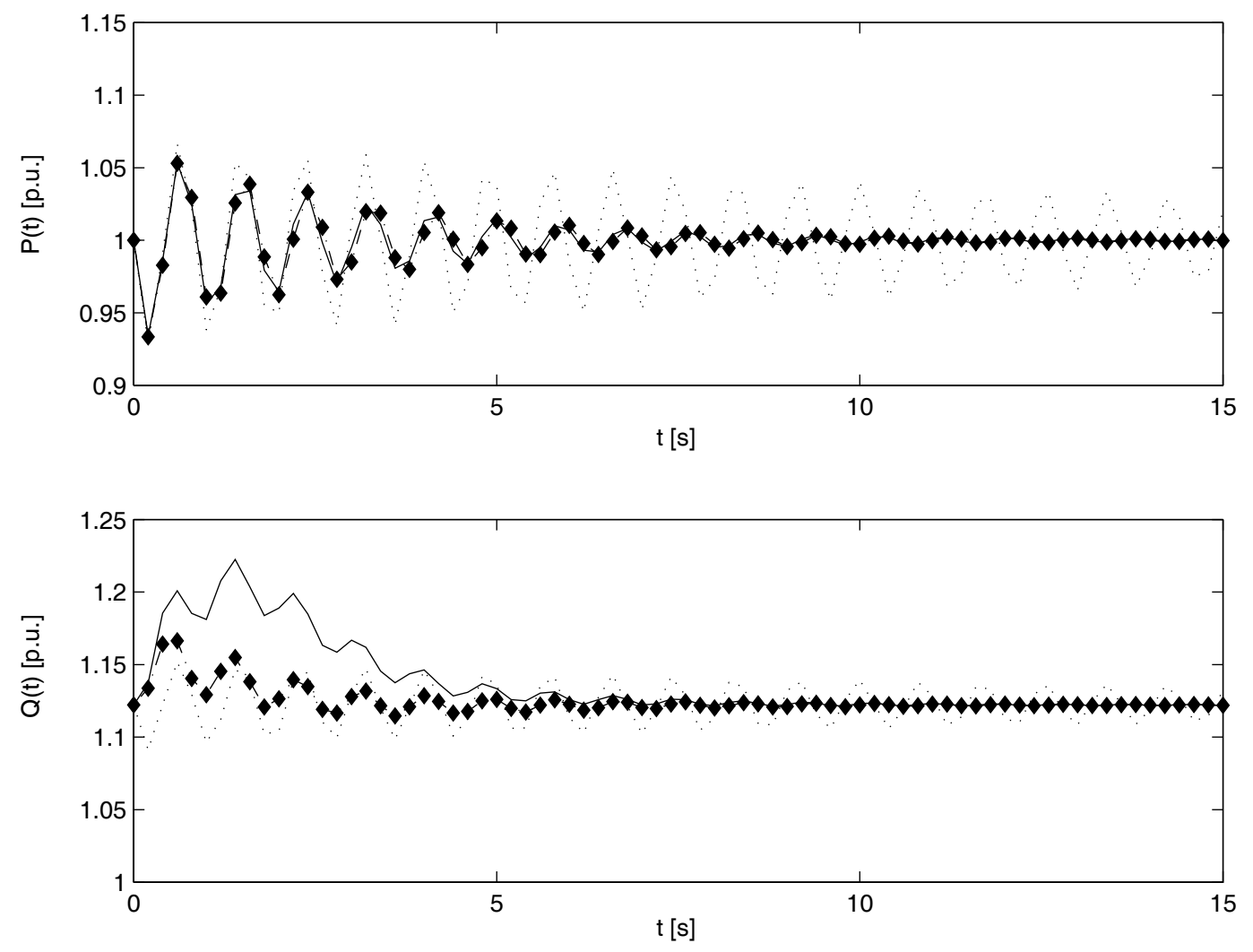

Figura 5.12: Caso 1 - Potências ativa e reativa: '-' sistema MBI com controle fuzzy, '...' sistema MBI com PSS clássico e '- $\diamond$-' SFSM com controle fuzzy. 

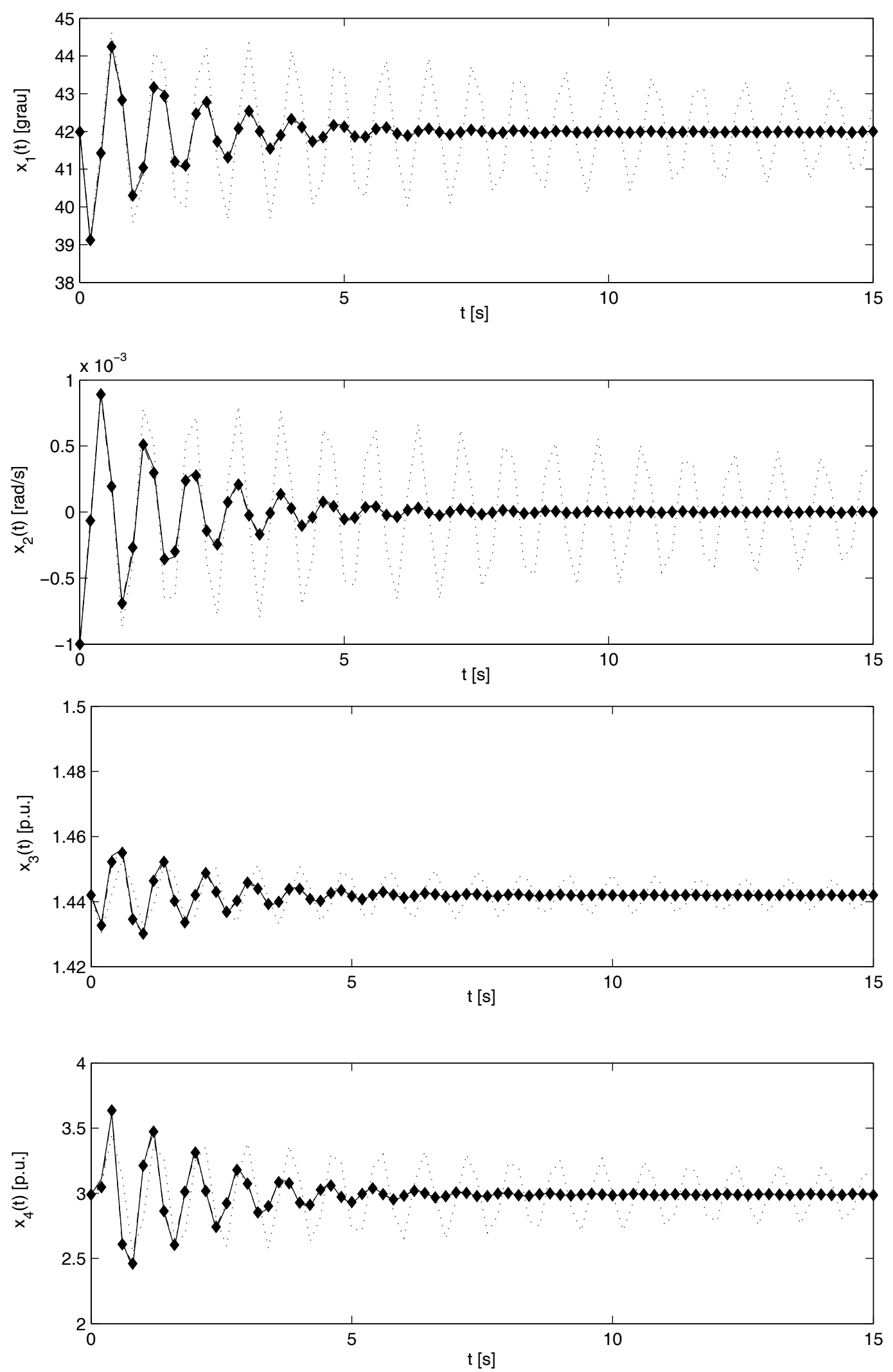

Figura 5.13: Caso 2 - Variáveis de estado: '-' sistema MBI com controle fuzzy, '..' sistema MBI com PSS clássico e '-॰-' SFSM com controle fuzzy. 

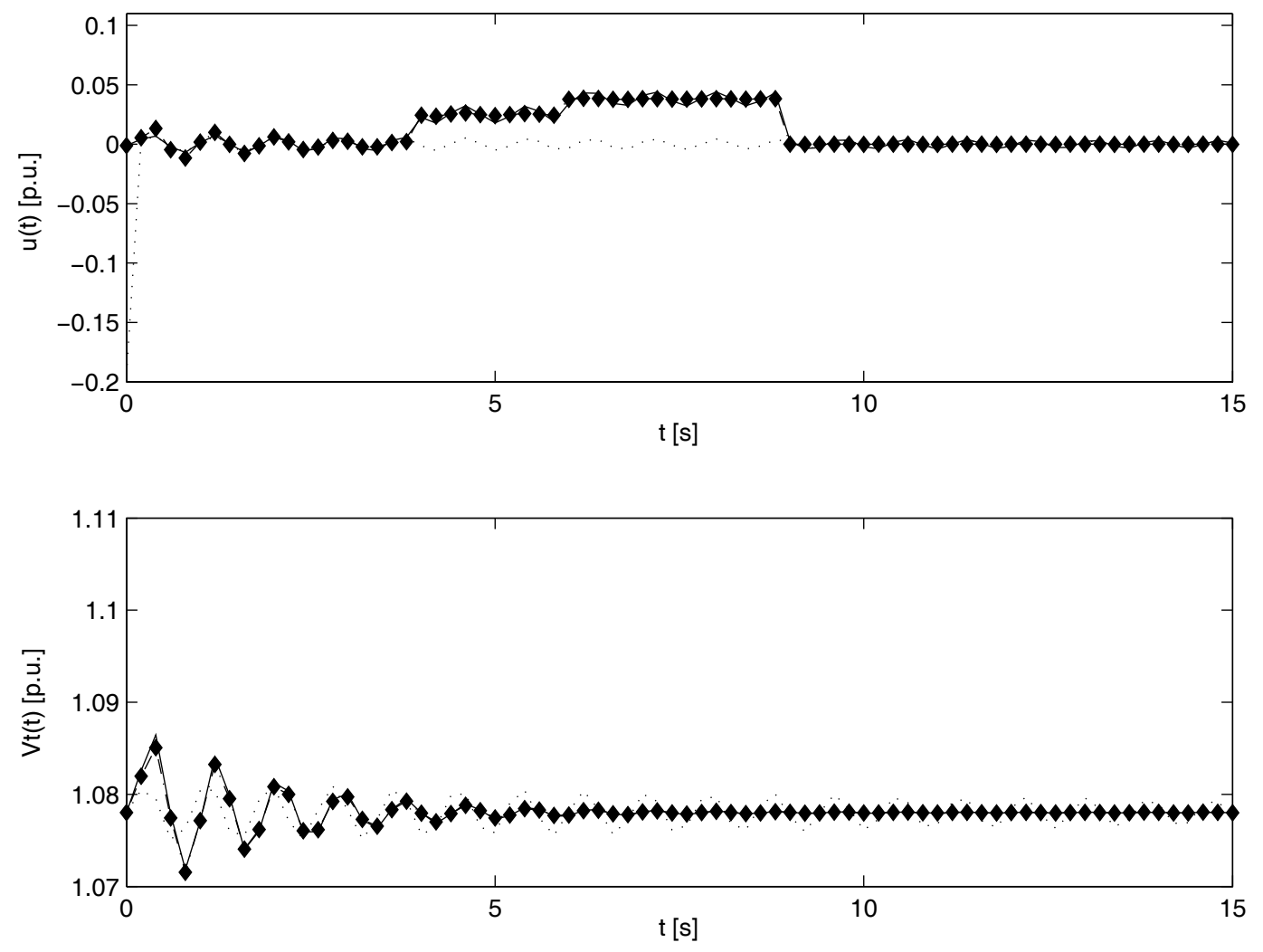

Figura 5.14: Caso 2 - Entrada de controle e tensão terminal: '-' sistema MBI com controle fuzzy, '...' sistema MBI com PSS clássico e '- $\diamond$ ' SFSM com controle fuzzy. 

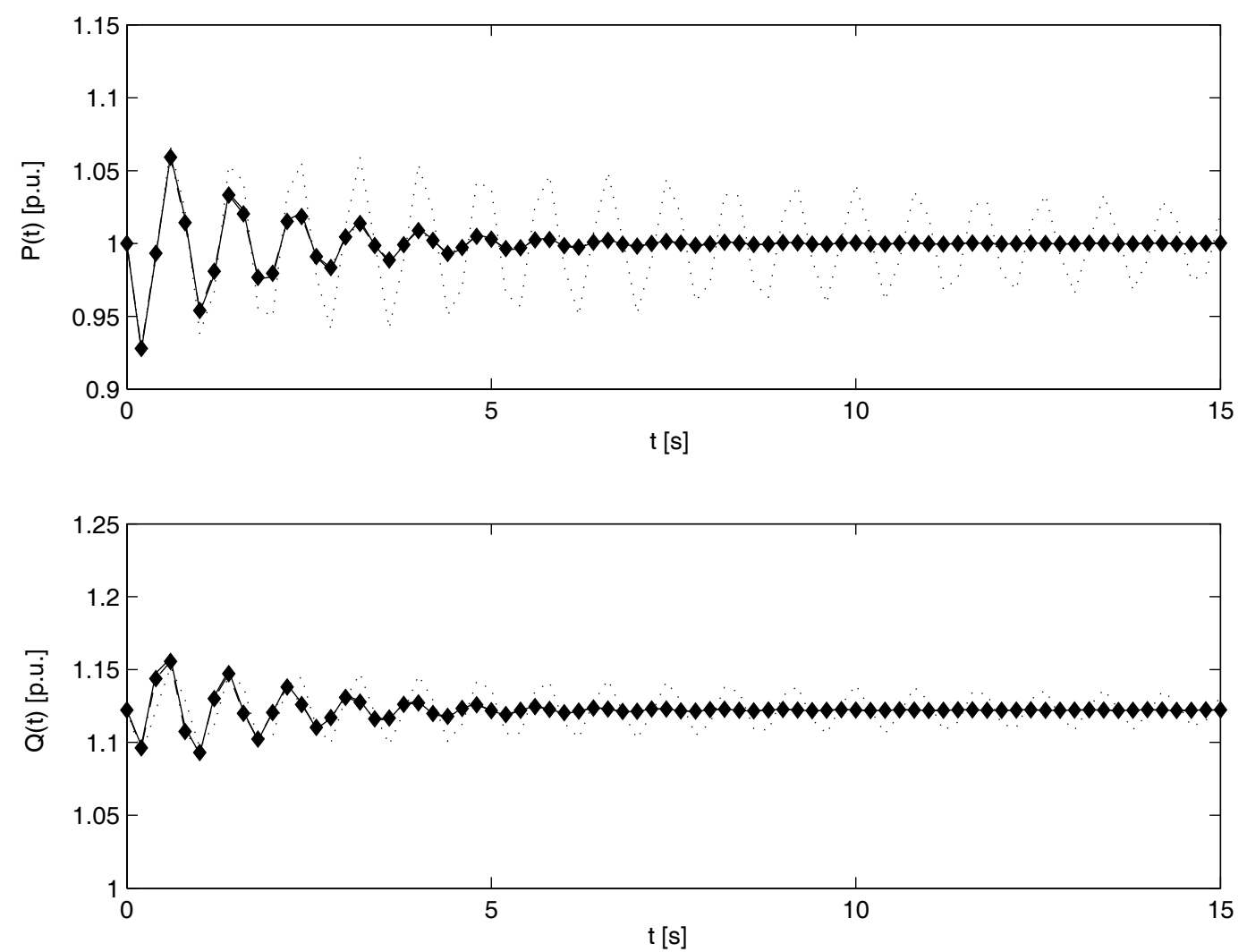

Figura 5.15: Caso 2 - Potências ativa e reativa: '-' sistema MBI com controle fuzzy, '...' sistema MBI com PSS clássico e '- $\diamond$ ' SFSM com controle fuzzy. 

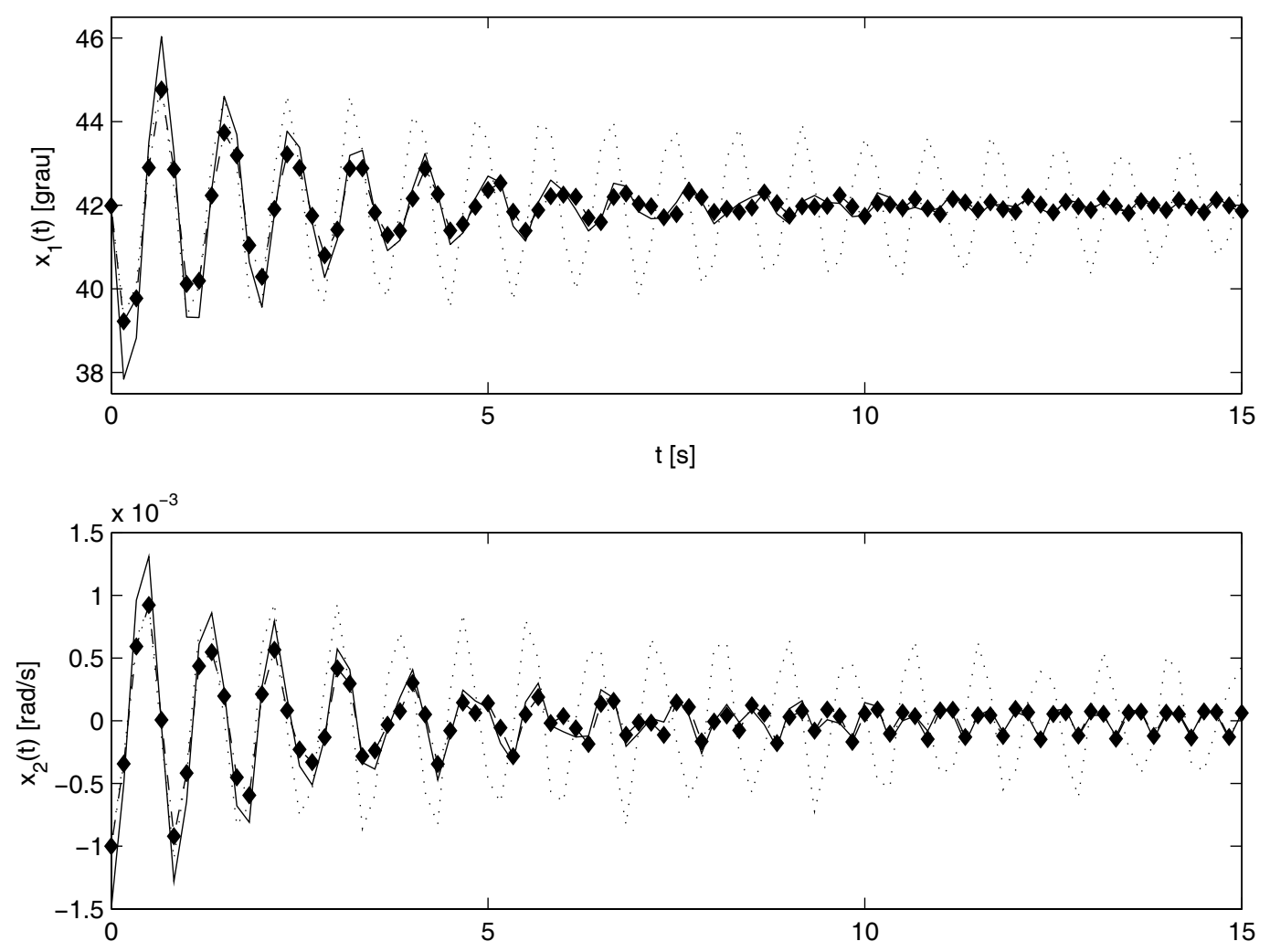

$\mathrm{t}$ [s]
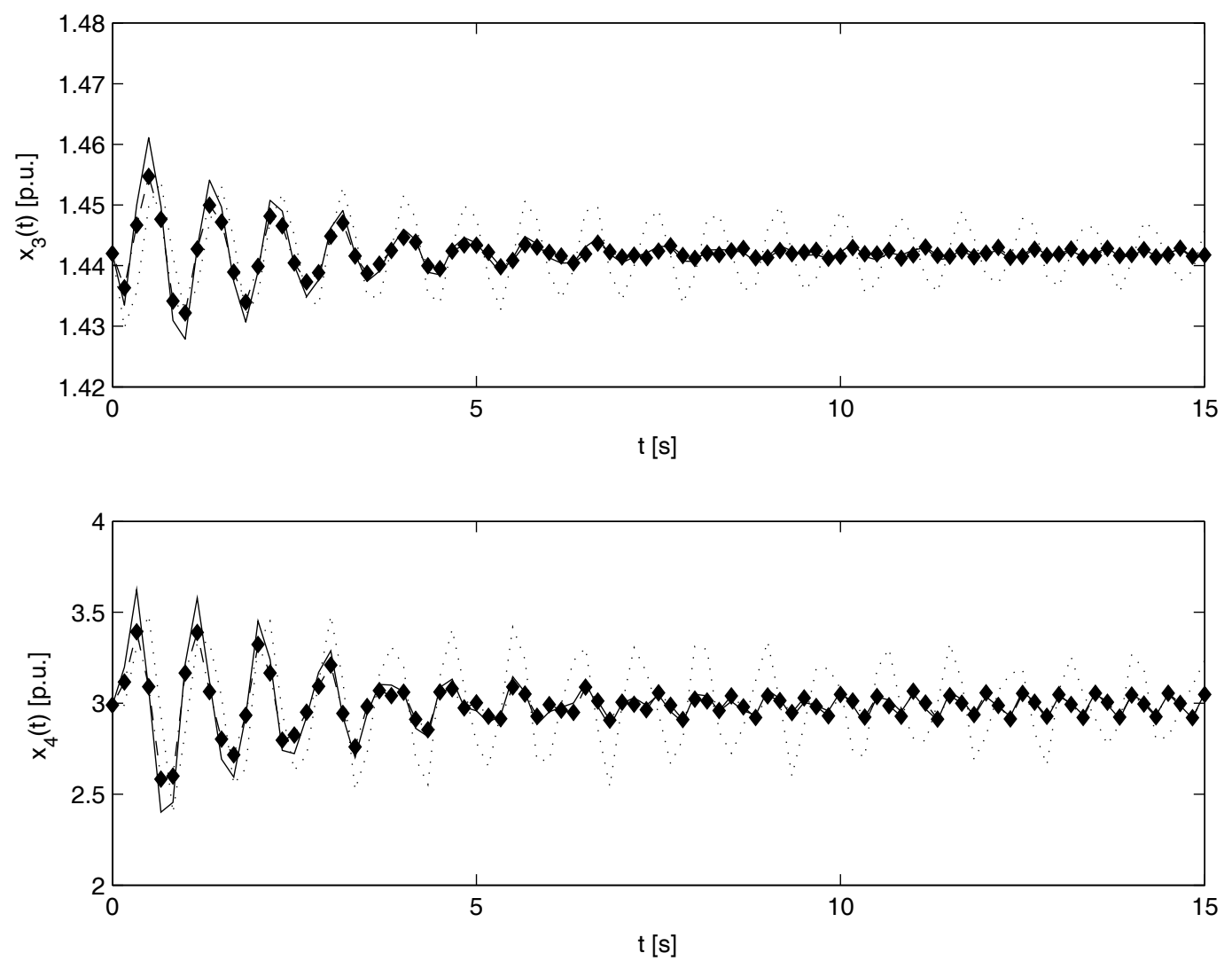

Figura 5.16: Caso 3 - Variáveis de estado: '-' sistema MBI com controle fuzzy, '...' sistema MBI com PSS clássico e '-^-' SFSM com controle fuzzy. 

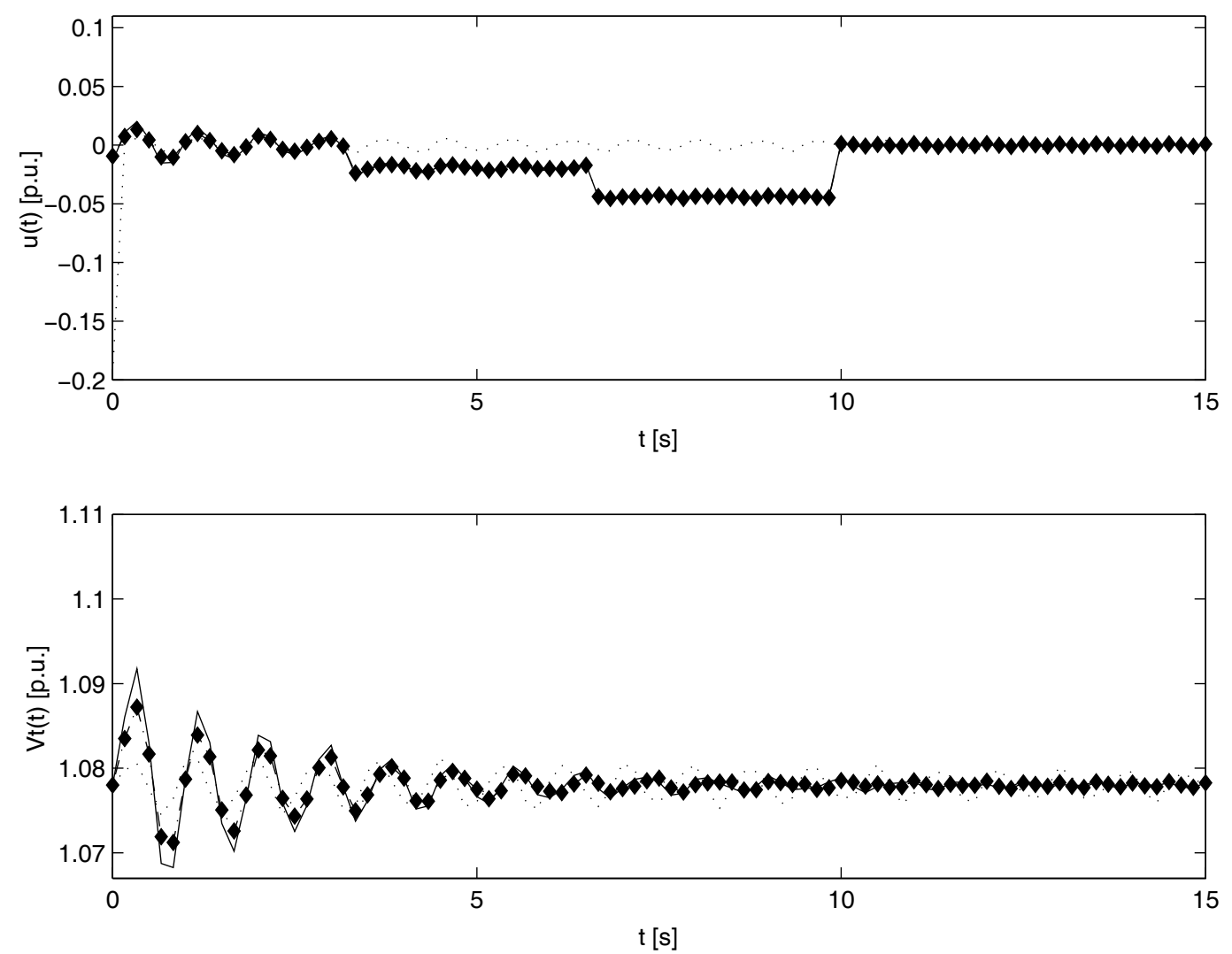

Figura 5.17: Caso 3 - Entrada de controle e tensão terminal: '-' sistema MBI com controle fuzzy, '...' sistema MBI com PSS clássico e '- $\diamond$-' SFSM com controle fuzzy. 


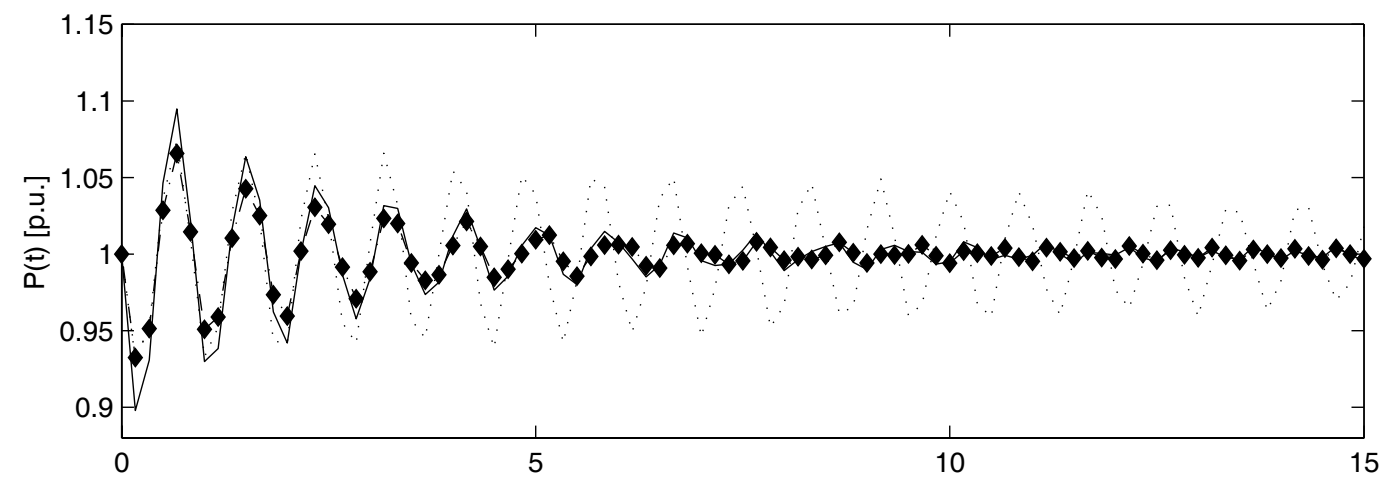

$\mathrm{t}[\mathrm{s}]$

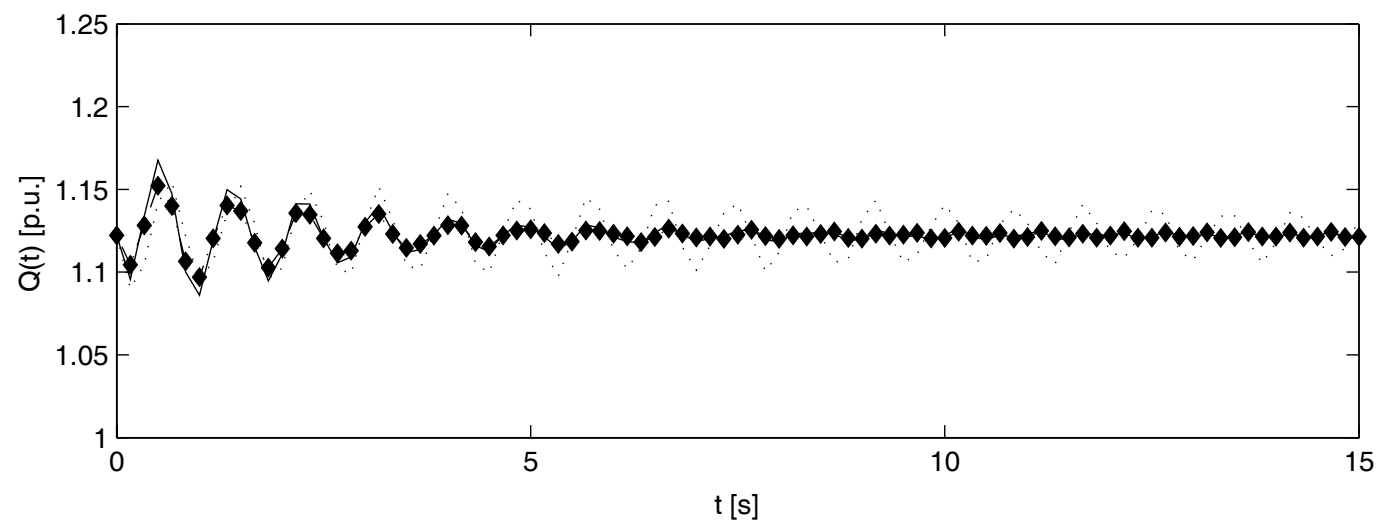

Figura 5.18: Caso 3 - Potências ativa e reativa: '-' sistema MBI com controle fuzzy, '...' sistema MBI com PSS clássico e '- $\diamond$ ' SFSM com controle fuzzy.

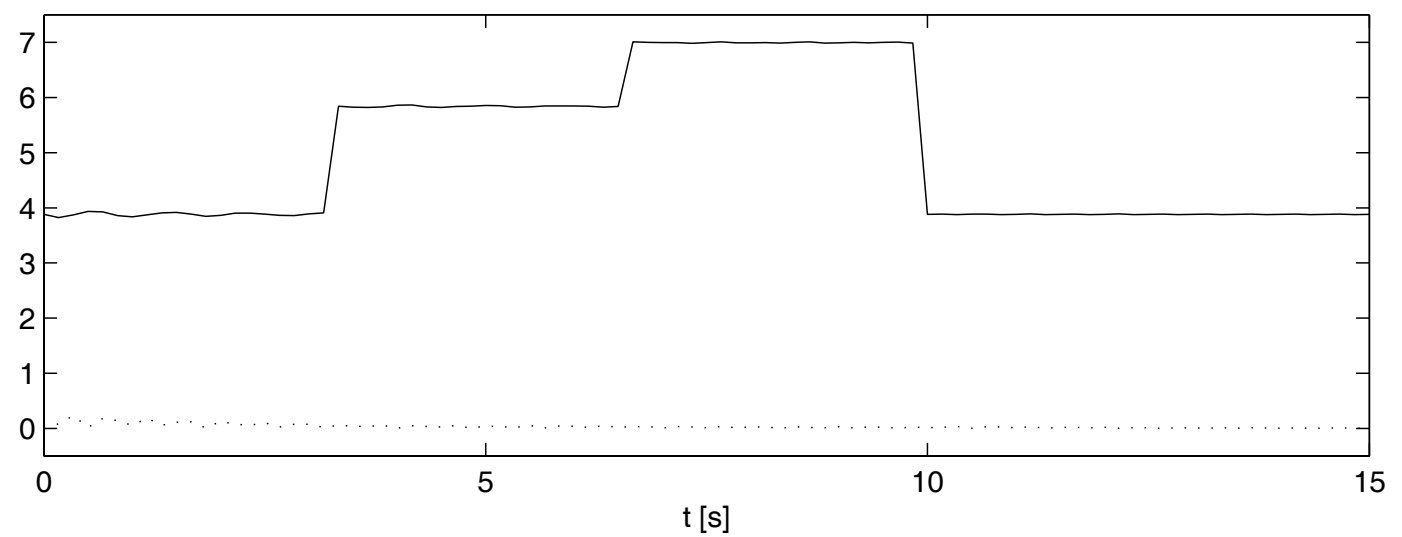

Figura 5.19: Erro de aproximação: '-' $\left\|\sum_{i=1}^{3} \sum_{j=1}^{2} m_{i}(z) n_{i j}(x) \Delta A_{i j} x\right\| \quad$ e '..' $\left\|f(x, r)-\sum_{i=1}^{3} \sum_{j=1}^{2} m_{i}(z) n_{i j}(x) A_{i j} x\right\|$ 


\section{Capítulo 6}

\section{Conclusão e Proposições para Trabalhos Futuros}

\subsection{Conclusão}

Neste trabalho foi apresentada uma abordagem fuzzy para a estabilização de uma classe de sistemas não-lineares com parâmetros descritos por saltos Markovianos. Esta classe de sistemas foi modelada por um sistema fuzzy com saltos Markovianos, cuja estrutura apresenta dois níveis: um nível que efetua transições abruptas definidas por conjuntos ordinários, representando o processo de saltos Markovianos e outro nível que efetua transições suaves definidas por conjuntos fuzzy, representando as não-linearidades no estado do sistema. A partir desta modelagem fuzzy e de conceitos de estabilidade e estabilização estocástica, foram elaboradas diferentes técnicas de projeto de controladores fuzzy. Estas técnicas incluem o conhecimento da operação do sistema e são computacionalmente tratáveis, utilizando técnicas de programação convexa atualmente disponíveis. Também, um exemplo de aplicação, caracterizando a operação de um sistema elétrico de potência em esquema de co-geração por um sistema não-linear com saltos Markovianos, foi desenvolvido para validação da abordagem proposta.

A abordagem fuzzy proposta apresentou resultados satisfatórios e promissores, uma vez que a dinâmica da classe de sistemas não-lineares considerada foi bem modelada tanto do ponto de vista quantitativo, pela representação em modelos lineares locais, quanto do ponto de vista qualitativo, pela inclusão de descrições lingüísticas da ope- 
ração do sistema. Ainda, outras diferentes técnicas de projeto de controladores fuzzy podem ser exploradas usando a modelagem fuzzy construída. Neste contexto, podem ser incluídas, por exemplo, incertezas paramétricas e ou distúrbios na modelagem, dentre outras variáveis que possam afetar o desempenho do controlador no sistema não-linear.

O inconveniente desta técnica é o crescente esforço computacional, visto que, os projetos de controladores fuzzy formulados consistem na busca de uma solução que atenda um conjunto de restrições descritas em LMI's, sendo que o número destas restrições está estritamente relacionado à quantidade de modos assumidos pelo processo de Markov e, também, ao número de regras de inferência fuzzy. No sentido de redução do esforço computacional ocasionado por um elevado número de regras de inferências fuzzy, condições LMI's mais relaxadas podem ser formuladas a partir de técnicas já disponíveis na literatura e que exploram as propriedades das funções de pertinência dos conjuntos fuzzy. Em relação a redução do conservadorismo da solução de controle que está baseada em uma função de Lyapunov acoplada do tipo $V(x, i)=x^{T} P_{i} x, P_{i}=P_{i}^{T}, P_{i}>0$, pode-se explorar uma função de Lyapunov fuzzy para obtenção de novos resultados de estabilização. Vale ressaltar que o aumento da complexidade matemática e computacional é comum em técnicas de controle não-linear. Por esse motivo, optou-se pelo desenvolvimento de técnicas de controle baseadas em LMI's, que podem ser computacionalmente implementadas e com razoável precisão usando programas disponíveis atualmente, como é o caso do módulo LMI Control, disponível no Matlab.

O exemplo de aplicação proposto foi elaborado a partir de uma investigação ampla da operação de um sistema de potência em esquema de co-geração, feita em cooperação com o Laboratório de Análise Computacional em Sistemas Elétricos do Dept ${ }^{\circ}$. Engenharia Elétrica da EESC/USP. Este exemplo inclui aspectos não considerados até então na operação do sistema de potência, que são as mudanças abruptas e aleatórias no carregamento. Neste contexto, vale ressaltar que a abordagem fuzzy proposta apresenta um resultado promissor no sentido de que a estabilização do sistema é realizada usando conceitos de estabilidade estocástica, diferente, portanto, das técnicas de controle atualmente utilizadas, onde a análise da estabilidade é feita em relação ao posicionamento dos pólos do sistema. 


\subsection{Propostas para Trabalhos Futuros}

No sentido de gerar futuras contribuições, no que segue são apresentadas algumas propostas para trabalhos futuros com base nos resultados obtidos até este ponto.

- Extensão dos resultados para o caso discreto;

- Desenvolvimento de condições relaxadas para o projeto de controle fuzzy estabilizante, explorando as propriedades das funções de pertinência dos conjuntos fuzzy;

- Desenvolvimento de condições que reduzam o conservadorismo, utilizando uma função de Lyapunov fuzzy;

- Desenvolvimento de um controle fuzzy dinâmico para estabilização do sistema de potência MBI, visando considerar informação incompleta do vetor de estado do sistema;

- Desenvolvimento de uma solução de controle descentralizada, visando a aplicação da abordagem proposta ao sistema de potência multi-máquinas;

- Desenvolvimento de uma nova abordagem fuzzy para sistemas de controle tolerante a falhas. 


\section{Apêndice $A$}

\section{Determinação dos Erros de}

\section{Aproximação}

\section{A.1 Representação dos Erros de Aproximação em Setores}

Considere os erros de aproximação (4.6) e (4.7), repetidos a seguir para fácil referência

$$
\Delta f=\sum_{i=1}^{N} m_{i}(z)\left[\sum_{j=1}^{R} n_{i j}(x)\left(f(x, r)-A_{i j} x\right)\right]
$$

$$
\Delta g=-\sum_{i=1}^{N} m_{i}(z)\left[\sum_{j=1}^{R} \sum_{k=1}^{R} n_{i j}(x) n_{i k}(x)\left(g(x, r)-B_{i j}\right) K_{i k} x\right]
$$

com $f(x, i): \mathbb{R}^{n} \times \mathbb{S} \rightarrow \mathbb{R}^{n}$ e $g(x, i): \mathbb{R}^{n} \times \mathbb{S} \rightarrow \mathbb{R}^{m}$ as funções não-lineares em cada modo e $A_{i j}$ e $B_{i j}$ as matrizes dos subsistemas da representação fuzzy correspondente.

Supondo que o modo do sistema no instante $t$ seja $i$, i.e., $r=i, i \in \mathbb{S}$, considere que existem matrizes $\Delta A_{i j}$ e $\Delta B_{i j}, j=1,2, \ldots, R$, tais que

$$
\|\Delta f\| \leq\left\|\sum_{j=1}^{R} n_{i j}(x) \Delta A_{i j} x\right\|
$$

$$
\|\Delta g\| \leq\left\|\sum_{j=1}^{R} \sum_{k=1}^{R} n_{i j}(x) n_{i k}(x) \Delta B_{i j} K_{i k} x\right\|
$$


ao longo da trajetória de $x$, descritas por $\Delta A_{i j}=\delta_{i j} A_{\mathbf{p} i}$ e $\Delta B_{i j}=\eta_{i j} B_{\mathbf{p} i}$, com $\left\|\delta_{i j}\right\| \leq 1$ e $\left\|\eta_{i j}\right\| \leq 1, j=1,2, \ldots, R$ e $A_{\mathbf{p} i}$ e $B_{\mathbf{p} i}$ matrizes limitantes de dimensões apropriadas. No que segue, apresenta-se um procedimento para obtenção das matrizes $\Delta A_{i j}$ e $\Delta B_{i j}$.

Tendo em vista a descrição em setores das funções $f(x, i)$ e $g(x, i)$ (Vidyasagar, 1993), define-se as seguintes restrições para as respectivas $k$-ésimas linhas de $f(\cdot, \cdot)$ e $g(\cdot, \cdot)$

$$
\left(1-\varphi_{i j k}\right) f_{i j k}^{L} \leq f_{i k} \leq\left(1+\varphi_{i j k}\right) f_{i j k}^{L}
$$

e

$$
\left(1-\phi_{i j k}\right) g_{i j k}^{L} \leq g_{i k} \leq\left(1+\phi_{i j k}\right) g_{i j k}^{L}
$$

onde $\varphi_{i j k}$ e $\phi_{i j k}, k=1,2, \ldots, n$ são constantes positivas, $f_{i j k}^{L}=\left[\begin{array}{llll}a_{k 1}^{i j} & a_{k 2}^{i j} & \ldots & a_{k n}^{i j}\end{array}\right] x \mathrm{e}$ $g_{i j k}^{L}=\left[\begin{array}{llll}b_{k 1}^{i j} & b_{k 2}^{i j} & \ldots & b_{k m}^{i j}\end{array}\right], \operatorname{com} a_{k s}^{i j}, s=1,2, \ldots, n$ e $b_{k q}^{i j}, q=1,2, \ldots, m$ os elementos das $k$-ésimas linhas das matrizes $A_{i j}$ e $B_{i j}$, respectivamente. De (A.5) e (A.6), pode-se obter os seguintes valores máximos $\bar{\varphi}_{i j k}$ e $\bar{\phi}_{i j k}$ para as constantes $\varphi_{i j k}$ e $\phi_{i j k}$, respectivamente,

$$
\bar{\varphi}_{i j k}=\max _{x \in \mathbb{X}, f_{i j k}^{L} \neq 0}\left|\frac{f_{i j k}}{f_{i j k}^{L}}-1\right|
$$

e

$$
\bar{\phi}_{i j k}=\max _{x \in \mathbb{X}, g_{i j k}^{L} \neq 0}\left|\frac{g_{i j k}}{g_{i j k}^{L}}-1\right| .
$$

Deste modo, obtém-se as matrizes limitantes

$$
\Delta A_{i j}=\left[\Delta a_{k s}^{i j}\right]_{k, s=1,2, \ldots, n}
$$

e

$$
\Delta B_{i j}=\left[\Delta b_{k q}^{i j}\right]_{k=1,2, \ldots, n ; q=1,2, \ldots, m}
$$

$\operatorname{com} \Delta a_{k s}^{i j}=\bar{\varphi}_{i j k} a_{k s}^{i j}$ e $\Delta b_{k q}^{i j}=\bar{\varphi}_{i j k} b_{k q}^{i j}$. Então, para $-\epsilon_{\mathbf{a} k s} \leq \Delta a_{k s}^{i j} \leq \epsilon_{\mathbf{a} k s}$ e $-\epsilon_{\mathbf{b} k q} \leq$ $\Delta b_{k q}^{i j} \leq \epsilon_{\mathbf{b} k q}$, pode-se escrever

$$
\Delta A_{i j}=\operatorname{diag}\left\{\delta_{k s}^{i j}\right\}_{k, s=1,2, \ldots, n} A_{\mathbf{p} i}
$$


e

$$
\Delta B_{i j}=\operatorname{diag}\left\{\eta_{k q}^{i j}\right\}_{k=1,2, \ldots, n ; q=1,2, \ldots, m} B_{\mathbf{p} i}
$$

onde $-1 \leq \delta_{k s}^{i j} \leq 1,-1 \leq \eta_{k q}^{i j} \leq 1$,

$$
A_{\mathbf{p} i}=\left[\epsilon_{\mathbf{a} k s}\right]_{k, s=1,2, \ldots, n}
$$

e

$$
B_{\mathbf{p} i}=\left[\epsilon_{\mathbf{b} k q}\right]_{k=1,2, \ldots, n ; q=1,2, \ldots, m} .
$$

\section{A.2 Cálculo do erro de aproximação para o sistema de potência MBI em esquema de co-geração}

Considere o erro de aproximação entre o sistema de potência MBI em esquema de co-geração (5.24) e sua representação fuzzy (5.25) que é dado por

$$
\Delta f=\sum_{i=1}^{3} \sum_{j=1}^{2} m_{i}(z) n_{i j}(\xi)\left(f\left(\xi+x_{e}, r\right)-A_{i j} \xi\right)
$$

onde $\xi=x-x_{e}$ é o vetor de estado deslocado,

$$
\begin{aligned}
& f\left(\xi+x_{e}, i\right)=\left[\begin{array}{ll}
\omega_{0} & \left(\xi_{2}+x_{2 e}\right) \\
\frac{1}{2 H} & {\left[P_{m}-\left(\xi_{3}+x_{3 e}\right)\left(K_{1 q}(i) \cos \left(\xi_{1}+x_{1 e}\right)\right.\right.} \\
& \left.+K_{2 q}(i) \sin \left(\xi_{1}+x_{1 e}\right)+K_{3 q}(i)\left(\xi_{3}+x_{3 e}\right)\right] \\
\frac{1}{\tau_{d o}^{\prime}} & {\left[\left(\xi_{4}+x_{4 e}\right)-\left(\xi_{3}+x_{3 e}\right)+\left(x d-x_{d}^{\prime}\right)\left(K_{1 d}(i) \cos \left(\xi_{1}+x_{1 e}\right)\right.\right.} \\
& \left.\left.+K_{2 d}(i) \sin \left(\xi_{1}+x_{1 e}\right)+K_{3 d}(i)\left(\xi_{3}+x_{3 e}\right)\right)\right] \\
\frac{1}{T_{e}} & {\left[K_{e}\left(V_{r e f}(i)-\left|V_{t}\left(\xi+x_{e}, i\right)\right|\right)-\left(\xi_{4}+x_{4 e}\right)\right]}
\end{array}\right] \\
& \left|V_{t}\left(\xi+x_{e}, i\right)\right|=\left\{\left[\left(\xi_{3}+x_{3 e}\right)+x_{d}^{\prime}\left(K_{1 d}(i) \cos \left(\xi_{1}+x_{1 e}\right)+K_{2 d}(i) \sin \left(\xi_{1}+x_{1 e}\right)\right.\right.\right. \\
& \left.\left.+K_{3 d}(i)\left(\xi_{3}+x_{3 e}\right)\right)\right]^{2}+\left[x _ { d } ^ { \prime } \left(K_{1 q}(i) \cos \left(\xi_{1}+x_{1 e}\right)+K_{2 q}(i) \sin \left(\xi_{1}+x_{1 e}\right)\right.\right. \\
& \left.\left.\left.+K_{3 q}(i)\left(\xi_{3}+x_{3 e}\right)\right)\right]^{2}\right\}^{1 / 2} \text {. }
\end{aligned}
$$

e $A_{i j}, i=1,2,3, j=1,2$ são as matrizes dos lineares locais obtidas nos pontos de linearização $\bar{x}$ (veja Capítulo 5) 
modo 1:

$$
A_{11}=\left[\begin{array}{rrrr}
0 & 377 & 0 & 0 \\
-0.1378 & 0 & -0.0679 & -0.0011 \\
-0.1569 & 0 & -0.4695 & 0.1672 \\
1670 & 0 & -5326.3 & -101.08
\end{array}\right] ; A_{12}=\left[\begin{array}{rrrr}
0 & 377 & 0 & 0 \\
-0.1016 & 0 & -0.0814 & 0.0008 \\
-0.1853 & 0 & -0.4699 & 0.1662 \\
1898.1 & 0 & -5213.4 & -99.38
\end{array}\right] ;
$$

modo 2:

$$
A_{21}=\left[\begin{array}{rrrr}
0 & 377 & 0 & 0 \\
-0.1431 & 0 & -0.0634 & -0.0012 \\
-0.1542 & 0 & -0.4729 & 0.1672 \\
1676.5 & 0 & -5285 & -100.87
\end{array}\right] ; A_{22}=\left[\begin{array}{rrrr}
0 & 377 & 0 & 0 \\
-0.1046 & 0 & -0.0775 & 0.0009 \\
-0.1825 & 0 & -0.4733 & 0.1662 \\
1901.9 & 0 & -5175.9 & -99.57
\end{array}\right] ;
$$

modo 3:

$$
A_{31}=\left[\begin{array}{rrrr}
0 & 377 & 0 & 0 \\
-0.1556 & 0 & -0.0540 & -0.0016 \\
-0.1490 & 0 & -0.4795 & 0.1672 \\
1692.4 & 0 & -5204.4 & -100.26
\end{array}\right] ; A_{32}=\left[\begin{array}{rrrr}
0 & 377 & 0 & 0 \\
-0.1111 & 0 & -0.0693 & 0.0011 \\
-0.1771 & 0 & -0.4798 & 0.1662 \\
1910.9 & 0 & -5102.6 & -100.10
\end{array}\right] \text {. }
$$

Então, considerando variações de $\xi_{1}$ no intervalo de $[-0.1,0.1]$ para cada modo e usando (A.7), obtém-se as seguintes matrizes $\Delta A_{i j}, i=1,2,3, j=1,2$

modo 1:

$$
\Delta A_{11}=\left[\begin{array}{rrrr}
0 & 0 & 0 & 0 \\
-0.0001 & 0 & -0.0001 & 0 \\
-0.0002 & 0 & 0 & 0 \\
0.2625 & 0 & -4.2997 & -0.0847
\end{array}\right] ; \Delta A_{12}=\left[\begin{array}{rrrr}
0 & 0 & 0 & 0 \\
-0.0001 & 0 & -0.0001 & 0 \\
-0.0002 & 0 & 0 & 0 \\
0.2782 & 0 & -5.5409 & -0.1158
\end{array}\right] ;
$$

modo 2 :

$\Delta A_{21}=\left[\begin{array}{rrrr}0 & 0 & 0 & 0 \\ -0.0001 & 0 & -0.0001 & 0 \\ -0.0002 & 0 & 0 & 0 \\ 0.2814 & 0 & -3.5476 & -0.0702\end{array}\right] ; \Delta A_{22}=\left[\begin{array}{rrrr}0 & 0 & 0 & 0 \\ -0.0001 & 0 & -0.0001 & 0 \\ -0.0002 & 0 & 0 & 0 \\ 0.3051 & 0 & -4.6306 & -0.0972\end{array}\right] ;$

modo 3:

$\Delta A_{31}=\left[\begin{array}{rrrr}0 & 0 & 0 & 0 \\ -0.0002 & 0 & -0.0001 & 0 \\ -0.0001 & 0 & 0 & 0 \\ 0.2727 & 0 & -2.3121 & -0.0462\end{array}\right] ; \Delta A_{22}=\left[\begin{array}{rrrr}0 & 0 & 0 & 0 \\ -0.0001 & 0 & -0.0001 & 0 \\ -0.0002 & 0 & 0 & 0 \\ 0.3086 & 0 & -3.1297 & -0.0663\end{array}\right]$

a partir das quais, usando (A.11) e (A.13), resulta 
modo 1:

$A_{\mathbf{p} 1}=\left[\begin{array}{rrrr}0 & 0 & 0 & 0 \\ 0.0001 & 0 & 0.0001 & 0 \\ 0.0002 & 0 & 0 & 0 \\ 0.2782 & 0 & 5.5409 & 0.1158\end{array}\right] ;$

modo 2:

$$
A_{\mathbf{p} 2}=\left[\begin{array}{rrrr}
0 & 0 & 0 & 0 \\
0.0001 & 0 & 0.0001 & 0 \\
0.0002 & 0 & 0 & 0 \\
0.3051 & 0 & 4.6306 & 0.0972
\end{array}\right]
$$

modo 3:

$$
A_{\mathbf{p} 3}=\left[\begin{array}{rrrr}
0 & 0 & 0 & 0 \\
0.0001 & 0 & 0.0001 & 0 \\
0.0002 & 0 & 0 & 0 \\
0.3086 & 0 & 3.1297 & 0.0663
\end{array}\right] .
$$




\section{Bibliografia}

ARRIFANO, N. S. D.; OLIVEIRA, V. A. (2002). Synthesis of an LMI-based fuzzy control system with guaranteed cost performance: a piecewise approach, Anais do XIV Congresso Brasileiro de Automática, Natal, RN, p.2981-2986.

ARRIFANO, N. S. D.; OLIVEIRA, V. A. (n.d.a). Guaranteed cost fuzzy controllers for a class of uncertain nonlinear dynamic systems, Special issue on Dynamics, Control and Their Applications - Computational and Applied Mathematics, v. A aparecer.

ARRIFANO, N. S. D.; OLIVEIRA, V. A. (n.d.b). State feedback fuzzy-model-based control for Markovian jump nonlinear systems, Revista Controle $\mathfrak{E}$ Automação $S B A$, v. A aparecer.

ARRIFANO, N. S. D.; OLIVEIRA, V. A.; RAMOS, R. A. (2004). Fuzzy stabilization of power systems subject to random abrupt variations of operating conditions, Proc. American Control Conference - ACC.

BOUKAS, E. K.; LIU, Z. K. (2001). Suboptimal design of regulators for jump linear system with time-multiplied quadratic cost, IEEE Trans. Automatic Control, v.46, n.1, p.944-949.

BOUKAS, E. K.; LIU, Z. K.; AL-SUNNI, F. (1999). Guaranteed cost control of Markov jump uncertain system with time-multiplied cost function, Proc. 38th Conference Decision and Control, p.4125-4130.

BOUKAS, E. K.; SHI, P.; NGUANG, S. K. (2003). Robust $\mathcal{H}_{\infty}$ control for linear Markovian jump systems with unknown nonlinearities, J. Mathematics and Applications, v.282, p.241-255. 
BOUKAS, E. K.; YANG, H. (1999a). Exponential stabilizability of stochastic systems with Markovian jump parameters, Automatica, v.35, n.9, p.1437-1441.

BOUKAS, E. K.; YANG, H. (1999b). Robust lq regulator for jump linear systems with uncertain parameters, Dynamic Control, v.9, n.2, p.125-134.

BOYD, S.; GHAOUI, L. E.; FERON, E.; BALAKRISHNAN, V. (1994). Linear matrix inequalities in system and control theory, Philadelphia, PA: SIAM.

BRETAS, N. G.; ALBERTO, L. F. C. (1999). Estabilidade transitória em sistemas eletroenergéticos, São Carlos, SP: EESC-USP.

CHEN, B. S.; TSENG, C. S.; UANG, H. J. (1999). Robustness design of nonlinear dynamic systems via fuzzy linear control, IEEE Trans. Fuzzy Systems, v.7, n.5, p. $571-585$.

CHEN, H. F. (1980). On stochastic observability and controllability, Automatica, v.16, p.179-190.

CHUANJIANG, Z.; KHAMMASH, M.; VITTAL, V.; QIU, W. (2003). Robust power system stabilizer design using $\mathcal{H}_{\infty}$ loop shaping approach, IEEE Trans. Power Systems, v.18, n.2, p.810-818.

COLANERI, P.; GEROMEL, J. C.; LOCATELLI, A. (1997). Control theory and design, Academic Press.

COSTA, E. F. (2002). Detetabilidade de sistemas lineares sujeitos a saltos Markovianos, Tese (Doutorado), Universidade Estadual de Campinas, Faculdade de Engenharia Elétrica e de Computação.

COSTA, E. F.; DO VAL, J. B. R. (2002). Weak detectability and the linear quadratic control problem of discrete-time Markov jump linear systems, I. J. Control, v.75, n.16, p.1282-1292.

COSTA, O. L. V.; BOUKAS, E. K. (1998). Necessary and sufficient condition for robust stability and stabilizability of continuous-time linear systems with Markovian jumps, I. J. Optimization Theory and Applications, v.99, n.2, p.359-379.

CREDER, H. (1995). Instalações elétricas, Rio de Janeiro: L.C.T. 
DEMELLO, F. P.; CONCORDIA, C. (1969). Concept of synchronous machine stability as affected by excitation control, IEEE Trans. Power Apparatus and Systems, v.PAS-88, n.4, p.316-329.

DUGAN, R. C.; MCGRANAGHAN, M. F.; BEATY, H. W. (1996). Electrical power systems quality, McGraw-Hill.

FANG, Y.; LOPARO, K. A. (2002). Stochastic stability of jump linear systems, IEEE Trans. Automatic Control, v.47, n.7, p.1204-1208.

FARIAS, D. P.; GEROMEL, J. C.; DO VAL, J. B. R.; COSTA, O. L. V. (2000). Output feedback control of Markov jump linear systems in continuous-time, IEEE Trans. Automatic Control, v.45, n.5, p.944-949.

FENG, X.; LOPARO, K. A.; JI, Y.; CHIZECK, H. J. (1992). Stochastic stability properties of jump linear systems, IEEE Trans. Automatic Control, v.37, n.1, p.3853.

FITZGERALD, A. E.; KINGSLEY, C. J. (1975). Máquinas elétricas, McGraw-Hill do Brasil.

GAHINET, P.; NEMIROVSKY, A.; LAUB, A. J.; CHILALI, M. (1995). LMI control toolbox for use with Matlab, Natick, MA: The Mathworks Inc.

GUO, Y.; HILL, D. J.; WANG., Y. (2000). Nonlinear decentralized control of large-scale power systems, Automatica, v.36, p.1275-1289.

GUO, Y.; HILL, D. J.; WANG., Y. (2001). Global transient stability and voltage regulation for power systems, IEEE Trans. Power Systems, v.16, n.4, p.678-688.

HONG, S. K.; LANGARI, R. (1998). Synthesis of an LMI-based fuzzy control system with guaranteed optimal $\mathcal{H}_{\infty}$ performance, IEEE International Conference on Fuzzy Systems Proc., v. 1, p.422-427.

HONG, S. K.; LANGARI, R. (2000). An LMI-based $\mathcal{H}_{\infty}$ fuzzy control system design with ts framework, Int. J. Information Sciences, v.123, p.163-169.

JADBABAIE, A.; JAMSHIDI, M.; TITLI, A. (1998). Guaranteed-cost design of continuous-time takagi-sugeno fuzzy controllers via linear matrix inequalities, The 1998 IEEE Int. Conf. Fuzzy Systems Proc., v. 1, p.422-427. 
JI, Y.; CHIZECK, H. J. (1990). Controllability, stabilizability, and continuous-time Markovian jump linear quadratic control, IEEE Trans. Automatic Control, v.35, n.7, p.777-788.

KAMKLA, J.; SOCHA, L. (1977). Some remarks about stochastic contollability, IEEE Trans. Automatic Control, v.AC-22, p.880-881.

KHALIL, H. (1996). Nonlinear systems, USA: Macmillan Publishing Company.

KRASOVSKII, N. N.; LIDSKII, E. A. (1961). Analytical design of controllers in systems with random attributes i, ii, iii, Automation Remote Control, v.22, p.1021-1025, 1141-1146, 1289-1294.

KUNDUR, P. (1994). Power system stability and control, McGraw-Hill.

KUSHNER, H. J. (1967). Stochastic stability and control, New York: Academic.

LARSEN, E. V.; SWANN, D. A. (1981). Applying power system stabilizers, P-III, IEEE Trans. Power Apparat. Syst., v.PAS-100, p.3017-3046.

LI, J.; WANG, O. H.; NIEMANN, D.; TANAKA, K. (2000). Dynamic parallel distributed compensation for Takagi-Sugeno fuzzy systems, Int. J. Information Sciences, v., n.123, p.201-221.

MACHADO, E. R. M. D.; TEIXEIRA, M. C. M.; ASSUNÇÃO, E. (2003). Construção e controle de modelos fuzzy Takagi-Sugeno reduzidos para sistemas não-lineares, Anais do VI Simpósio Brasileiro de Automática, Bauru, SP, p.876-881.

MAHMOUD, M. S.; SHI, P. (2002). Robust control for Markovian jump linear discretetime systems with unknown nonlinearities, IEEE Trans. Circuits and Systems I, v.49, n.4, p.538-542.

MALIK, O. P.; HOPE, G. S.; GORSKI, Y. M.; USHAKOV, V. A.; RACKEVICH, A. L. (1986). Experimental studies on adaptive microprocessor stabilizers for synchronous generators, IFAC Power System and Power Plant Control, p.125-130.

MAMDANI, E. H.; ASSILAN, S. (1975). An experiment in linguistic systems with a fuzzy logic controller, Int. J. Man Machine Studies, v.7, n.1, p.1-13.

MARITON, M. (1986). On controllability of linear systems with stochastic jump parameters, IEEE Trans. Automatic Control, v.AC-31, p.680-683. 
MARITON, M. (1990). Jump linear systems in automatic control, New York: Marcel Dekker.

MARK, F. K. (1992). Design of nonlinear generator excitors using differential geometric control theories, Proc. 31st IEEE Conf. Decision and Control, p.1149-1153.

NASCIMENTO, R. R.; OLIVEIRA, V. A.; ARRIFANO, N. S. D.; GESUALDO, E.; TOSETTI, J. P. V. (2002). Control of the molten steel level in a strip-casting process using fuzzy T-S models, Proc. 15th Triennial World Congress of the International Federation of Control, Barcelona, Spanish.

NESTEROV, Y.; NEMIROVSKY, A. (1994). Interior-point polynomial methods in convex programming, Philadelphia, PA: SIAM.

OLIVEIRA, V. A.; ARRIFANO, N. S. D. (2001). Controle de sistemas incertos, In: BAlthaZAR, J. M.; OliVEIRA, V. A.; SILVA, G. N.; ROSÁRIO, J. M., Aplicações em dinâmica e controle, São Carlos, SP: ABCM - SBMAC, Cap. 14, p.326349.

OLIVEIRA, V. A.; ARRIFANO, N. S. D. (2003). Controle de sistemas não-lineares baseado em modelagem fuzzy, Anais do II Congresso Temático de Dinâmica, Controle e Aplicações, São José dos Campos, SP, v. 2,Série Arquimédes, p.1818-1850.

OLIVEIRA, V. A.; NASCIMENTO, R. R.; ARRIFANO, N. S. D.; GESUALDO, E.; TOSETTI., J. P. V. (2003). Non-linear control strategy for strip casting process, Revista da Sociedade Brasileira de Redes Neurais-SBRN. Disponível em: $<$ http://www.sbrn.org.br/fls/volume2/artigo1.pdf >

OTHMAN, H.; SANCHEZ-GASCA, J. J. (1989). On the design of robust power system stabilizers, Proc. 28th. Conf. Decision and Control, p.1853-1857.

PAPOULIS, A. (1984). Probability, random variables, and stochastic process, Singapore: McGraw-Hill Int. Edition.

RAO, P. S.; SEN, I. (1999). Robust tuning of power system stabilizers using QFT, IEEE Trans. Control System Technology, v.7, p.478-486.

RISHEL, R. (1975). Dynamic programming and minimum principles for systems with jump Markov disturbances, SIAM J. Control, v.13, n.2, p.338-371. 
SHAW, I. S.; OES, M. G. S. (1999). Controle e modelagem fuzzy, São Paulo: Edgard Blücher.

SWORDER, D. D. (1969). Feedback control of a class of linear systems with jump parameters, IEEE Trans. Automatic Control, v.AC-14, n.1, p.9-14.

SWORDER, D. D.; ROBINSON, V. G. (1973). Feedback regulators for jump parameter systems with control and state dependent transition rates, IEEE Trans. Automatic Control, v.AC-18, p.355-360.

TAKAGI, T.; SUGENO, M. (1985). Fuzzy identification of systems and its application to modeling and control, IEEE Trans. Systems, Man and Cybernetic, v.15, n.1, p.116-132.

TANAKA, K.; HORI, T.; WANG, H. O. (2003). A multiple Lyapunov function approach to stabilization of fuzzy control systems, IEEE Trans. Fuzzy Systems, v.11, n.4, p. $582-589$.

TANAKA, K.; IKEDA, T.; WANG, H. O. (1996). Robust stabilization of a class of uncertain nonlinear systems via fuzzy control: quadratic stabilizability, $\mathcal{H}_{\infty}$ control theory and linear matrix inequalities, IEEE Trans. Fuzzy Systems, v.4, n.1, p.1-13.

TANAKA, K.; IKEDA, T.; WANG, H. O. (1998). Fuzzy regulators and fuzzy observers: relaxed stability conditions and LMI-based designs, IEEE Trans. Fuzzy Systems, v.8, n.2, p.250-265.

TANAKA, K.; IWASAKI, M.; WANG, H. O. (2000). Stability and smoothness conditions for switching fuzzy systems, Proc. American Control Conference, p.24742478.

TANAKA, K.; WANG, H. O. (2001). Fuzzy control systems design and analysis: a linear matrix inequality approach, New York: John Wiley and Sons.

TANIGUCHI, T.; TANAKA, K.; OHTAKE, H.; WANG, H. O. (2001). Model construction, rule reduction, and robust compensation for generalized form of TakagiSugeno fuzzy systems, IEEE Trans. Fuzzy Systems, v.9, n.4, p.525-538.

TEIXEIRA, M. C. M.; ASSUNÇÃO, E.; AVELlAR, R. G. (2003). On relaxed LMIbased designs for fuzzy regulators and fuzzy observers, IEEE Trans. Fuzzy Systems, v.11, n.5, p.613-623. 
TEIXEIRA, M. C. M.; PIETROBOM, H. C.; ASSUNÇÃO, E. (2000). Novos resultados sobre a estabilidade e controle de sistemas não-lineares utilizando modelos fuzzy e LMI, Revista Controle \& Automação, v.11, n.1, p.37-48.

TEIXEIRA, M. C. M.; ŻAK, S. H. (1999). Stabilizing controller design for uncertain nonlinear systems using fuzzy models, IEEE Trans. Fuzzy Systems, v.15, n.1, p.116-132.

VIDYASAGAR, M. (1993). Nonlinear systems analysis, Prentice-Hall International.

WANER, S.; COSTENOBLE, S. R. (1997). Markov systems, Markov system simulation. Acesso em: 10 set. 2002. Disponível em: $<$ http://people.hofstra.edu/faculty/StefanWaner/RealWorld/markov/markov $>$

WANG, H. O.; TANAKA, K.; GRIFFIN, M. F. (1996). An approach to fuzzy control of nonlinear: stability and design issues, IEEE Trans. Fuzzy Systems, v.4, n.1, p.14-23.

WANG, L. X. (1997). A course in fuzzy systems and control, New Jersey: Prentice-Hall, Inc.

WANG, Y.; GUO, G.; HILL, D. J. (1997). Robust decentralized nonlinear controller design for multimachine power systems, Automatica, v.33, n.9, p.1725-1733.

WANG, Y.; HILL, D. J. (1996). Robust nonlinear coordinated control of power systems, Automatica, v.32, n.4, p.611-618.

WANG, Y.; HILL, D. J.; MIDDLETON, R. H.; GAO, L. (1993). Transient stability enhancement and voltage regulation of power systems, IEEE Trans. Power Systems, v.8, n. 2, p. $620-627$.

WANG, Y.; XIE, L.; HILL, D. J.; MIDDLETON, R. H. (1992). Robust nonlinear controller design for transient stability enhancement of power systems, Proc. 31st IEEE Conf. Decision and Control, p.1117-1122.

WERNER, H.; KORBA, P.; YANG, T. C. (2003). Robust tuning of power system stabilizers using LMI-techniques, IEEE Trans. Control System Technology, v.11, n.1, p.147-152.

ZADEH, L. A. (1965). Fuzzy sets, Informat. Control, v., n.8, p.338-353. 
ZHOU, K.; DOYLE, J. C. (1998). Essentials of robust control, Uppler Siddle River: Prentice-Hall, Inc.

ZHU, C.; ZHOU, R.; WANG, Y. (1997). A new nonlinear voltage controller for power systems, Electrical Power $\&$ Energy Systems, v.19, n.1, p.19-27. 